\title{
Annotazioni su B1,1-3 (B1,4a?) di Parmenide
}

\section{Vittorio Ricci}

The extraordinary overall textual situation of Parmenides' B1,1-3, due to complex, variegate and polymorphous causes, entailed and still entails diverse sorts of problematic issues so to constitute a true labyrinth of philological, hermeneutical and theoretical instances interwoven each other in almost inextricable way. In this analysis, a first substantial knot of philological type resulted necessary to a preliminary discrimination for making sure the textual reconstruction in order to argue then its most literarily clear and specifiable meaning. In this way it was also possible to make sure its semantic and theoretical relevancies. This research leaded to outline and, hopefully, to demonstrate no textual corruption and following misunderstanding happened before sec. XIII, namely corresponding to the lecture present in the first available manuscript, the so-called cod. N, universally excluded and entirely misevaluated, even discarded; instead it is not only the principle, but the unique reliable codex. The detailed analyse including the autoptic exam is the result of enlightening the absolute goodness of this version and the reasons both philological and hermeneutical comparations, which allow to achieve its complete textual rehabilitation and so to grasp its real conceptual content.

\section{Introduzione}

È noto che il proemio parmenideo concentra in sé una serie di problematiche teoretiche ed ermeneutiche ${ }^{1}$, oltre ad alcune questioni filologiche divenute quasi

\footnotetext{
1 Non si può nemmeno ipotizzare di esporre in tale sede una sintesi delle prospettive ermeneutiche riguardanti la valenza o le valenze del proemio parmenideo. Il proemio che rimane comunque notevolmente 'enigmatico', è stato schematizzato sulla base dell'opposizione anabasi/catabasi. Come anabasi hanno interpretato: H. Diels, Parmenides Lehrgedicht, Berlin 1897, 14; W. J. Verdenius, "Parmenides' Conception of Light", Mnemosyne 2, 1949, 119-120; F. M. Cornford, Principium sapientiae: The Origins of Greek Philosophical Thought, Cambridge University Press 1952, 118; K. Deichgräber, Parmenides Auffahrt zur Göttin des Rechts. Untersuchungen zum Proömion seines Lehrgedichts, Wiesbaden Steiner 1958, 33; E. F. Dolin, "Parmenides and Hesiod", HSPh 66, 1962, 93-98; W. K. C. Guthrie, A History of Greek Philosophy, vol. 2: The Presocratic Tradition from Parmenides to Democritus, Cambridge, Cambridge University Press 1965, 11; J. Mansfeld, Die Offenbarung des Parmenides und die menschliche Welt, Assen 1964, 234-247; H. Pfeiffer, Die Stellung des parmenideischen Lehrgedichtes in der epischen Tradition, Bonn, 1975; H. E. F. Fränkel, Early Greek Poetry and Philosophy, Oxford, Blackwell (traduzione inglese di M. Hadas et J. Willis), 1975, p. 365 ; D. B. Claus, Toward the Soul : An Inquiry into the Meaning of Psyche before Plato, New Haven, Yale University Press 1981; J. Bollack, Parménide, de l'étant au monde, Paris 2006 78; A. H. Coxon, The Fragments of Parmenides. A Critical Text with Introduction and Translation. The
} 
insolubili sin dai primi versi (specialmente in B1,3), anche a motivo di una trasmissione dossografica ingenerosa su più fronti che tuttavia non si è valutata nel modo più giusto. Pare opportuno innanzitutto rilevare che nel proemio parmenideo non si fa cenno a espressioni d'invocazione per il dono poetico o di richiesta di qualche soccorso divino perché sia ispirato o dettato ${ }^{2}$, né si indica qualche pubblico cui indirizzare quanto

Ancient Testimonia and a Commentary, Las Vegas, Parmenides Publishing, 2009, 276. Come interpreti in termini di katabasi si riscontrano: O. Gilbert, "Die $\delta \alpha i ́ \mu o v$ des Parmenides", AGPh 2, 1907, 25-45; J. S. Morrison, "Parmenides and Er", JHS 75, 1955, 59; L. TARAN, Parmenides. A Text with Translation, Commentary, and Critical Essays, Princeton University Press 1965, 24; W. Burkert, "Das Proömium des Parmenides und die "Katabasis" des Pythagoras", Phronesis 14, 1969, 7-9; M. E. Pellikaan-Engel, Hesiod and Parmenides : A New View on Their Cosmologies and on Parmenides' Proem, Amsterdam 1974, 51-53 et 60-62; R. J. Clark, Catabasis: Virgil and the Wisdom-Tradition, Amsterdam 1979, 33 e 49; J. Owens, "Knowledge and katabasis in Parmenides", The Monist 62, 1979, 15-29; A. P. D. Mourelatos, The Route of Parmenides, New Haven, Yale University Press 1970, 14-16 e 42-44; D. Gallop, Parmenides of Elea : Fragments, University of Toronto Press 1984, 6-7; M. M. Sassi, "Parmenide al bivio", PP 43, 1988, 383-396; D. J. Furley, Cosmic Problems : Essays on Greek and Roman Philosophy of Nature, Cambridge University Press 1989, 28; P. Kingsley, In the Dark Places of Wisdom, Londres 1999, 93-100; G. Cerri, Parmenide di Elea. Poema sulla natura, Milano 1999, 106-110; K. A. Morgan, Myth and Philosophy from the Presocratics to Plato, Cambridge University Press, 2000, 78; A. Hermann, To Think Like God: Pythagoras and Parmenides. The Origins of Philosophy, Las Vegas, Parmenides Publishing 2004, 166; L. Gemelli Marciano, "Images and Experience: At the Roots of Parmenides' Aletheia", AncPhil 28, 2008, 36; Y. Ustinova, Caves and the Ancient Greek Mind: Descending Underground in the Search for Ultimate Truth, Oxford University Press 2009, 201-202. Si è avanzata anche una prospettiva mediana, ovvero una realtà unica articolata da questi estremi di anabasi iperuranica o di katabasi ktonica o sotterranea, come in Gabriela Cursaru, "Le Proème de Parménide: anabase et /ou catabase ?", Cahiers des études anciennes, LIII-1, 39-63. [on line] URL: http:// etudesanciennes.revues.org/907, arrivagndo a sostenere verso la conclusione: "on remarque ainsi que l'ambigüité concernant la direction du voyage, soigneusement entretenue tout au long du Proème, est tout a fait précise: ici, comme ailleurs dans son Proème, Parménide a voulu s'écarter autant des schémas spécifiques de l'imagerie mythico-poétique, et surtout abolir le modèle dualiste qu'ils impliquent, en remplaçant l'opposition dualiste entre Lumière ou Jour et Nuit, anabase et catabase, par leur unité dialectique. Là, au point de contact entre les deux mondes, les opposes sont égaux, égalité de principe de deux éléments opposés : le rapport entre Lumière-Jour et Nuit, anabase et catabase, est celui d'une homologie accomplie. Le récit du voyage parménidien se polarise entre les deux membres du couple Lumière-Jour / Nuit qui se retrouvent problématiquement unifies dans le concept de l'être. Le Proème met explicitement en scène un dispositif reposant sur des paires contradictoires, mais l'opposition entre Lumière-Jour et Nuit, anabase et catabase, n'est qu'une surdétermination de leur unité" ( $i b$. [on line], 17). In effetti, è veramente difficile rintracciare note di tale "unité dialectique" o addirittura di "homologie accomplie". Non si tratta di nessuna verticalità direzionale polarizzata negli estremi opposti, ma di un'unica esclusiva direzionalità 'orizzontale' finora non perseguita, che comporta uno scoprimento di assoluta determinazione imbastito anche su un quasi obbligato preambolo di tipo fisico-cosmologico particolare.

${ }^{2} \mathrm{Si}$ potrebbe ritenere che l'intervento persuasivo delle giovani, prima di giungere alla soglia della dimora della dea, nell'invitare Dike ad aprirne la porta (B1,15-16) potrebbe essere assimilata alle altre espressioni invocative epiche, poetiche e religiose. Tuttavia è da rilevare che innanzitutto non si tratta propriamente d'invocazione, ma di persuasione con "dolci parole" non pronunciate dall'interessato in persona, ma dalle suddette guidatrici divine. Inoltre è da 
l'iniziato o il vate ha ricevuto, né ci si rivolge a un destinatario singolo o comunitario, nemmeno a un eventuale discepolo cui consegnare il componimento didascalico e degno di conservazione. ${ }^{3}$ Nel poema parmenideo l'unicità dell' "io" narrante è un referente alquanto 'passivo' che lo rende simile a privilegiati eletti a depositari di una verità superiore o divina, anzi dell'unica, solo perché gli esseri umani si sono inibiti tale tragitto gnoseologico (nella sua effettività noetico-metodologica) e non hanno risposto debitamente alla sua 'naturale' attivazione. Tuttavia la passività del кoṽ recezione neutra bensì dinamizzante e coinvolgente necessariamente una capacità razionale e critica di comprensione, che lo differenzia nettamente dagli altri autori chiamati a trasmettere per iscritto una verità ricevuta da qualche entità divina. Infatti, la dea parmenidea non rivela una verità umanamente inattingibile con le sole forze intellettive e cognitive del discente, ma perché sussiste anche per lui un reale rischio di smarrimento e di deviazione con la conseguenza di cadere nell'apparenza che si è imposta presso i mortali e soprattutto si è confusa con la verità $(B 8,60-61)$. Poiché 1"“io" parmenideo è comunque 'dipendente' da quello della "dea". Il proemio è letterariamente funzionale a introdurre una dimensione "interiore" (non intimistica), cioè una condizione di "ascolto" noetico (non solo e forse per niente religioso - il $\mu \tilde{v} \theta$ o $\varsigma$ che la dea rivolge al giovane idoneo si distacca radicalmente dalla forma mantica o mistica o epica $)^{4}$, ma anche comprensiva di uno scenario esteriore. Il dispositivo

evidenziare che la descrizione mitica non suggerisce la dinamica religiosa di una comunicazione di una rivelazione ispirata che scenda sul vate/iniziato/profeta o lo investa in qualche modo perché produca la sua opera per diffonderla, e nemmeno di un'elevazione (fisica) dell'interlocutore che deve ricevere il discorso vero percorrendo la sua unica via ancora mai percorsa da nessun mortale. Si tratta piuttosto di un metodo attraverso cui quanto genericamente o impropriamente si è configurato e si continua a configurare in genere, si viene a sapere nell'unico modo necessario e debito che sta per essere esposto dalla dea parmenidea.

${ }^{3}$ Il destinatario è l'interlocutore stesso che ha ricevuto la comunicazione autentica e infallibile della verità che non trema, e la sua eventuale diffusione non è limitata da qualche prescrizione misterico-esoterica, ma dipende dalle argomentazioni epidittico-logiche che la provano irrefutabilmente. Si è riconosciuto in qualche modo la discontinuità parmenidea con la tradizione e per es. sono accettabili le seguenti osservazioni: "tout en s'appropriant la tradition mythico-poétique, son imagerie et ses modes d'expression, Parménide la réorganise selon des critères nouveaux, l'intention fondamentalement didactique de son Poème reposant sur un processus de dissolution des références épiques nécessaires a la mise en évidence d'une nouvelle conception de la pensée, car l'enseignement qu'il livre vise bien plus l'instauration d'un savoir que la transmission d'une tradition" (Cursaru, art. cit., [on line] 17), benché non sia 1' "instauration d'un savoir", ma la determinazione gnoseologica appropriata dell'unica verità anche imprescindibilmente ormai in relazione a quella "tradition" e ai suoi esiti doxastici.

${ }^{4}$ Una lettura preminentemente teologizzante si può reperire in A. Drozdek, "Parmenides' Theology", Eranos XCIX (2001), 4-15, in cui si descrive la relatività per Parmenide della conoscenza umana - forse si dovrebbe enfatizzare la sua negatività assoluta - e l'attivazione del 
proemiale si connota di un'eccezionalità universalmente imparagonabile e intrinsecamente non assimilabile a qualsiasi altra umana, nella sua assolutezza originaria, originale e irripetibile. ${ }^{5}$ Nessuna nota storica, biografica, geografica è

logos da parte della dea per cui si conclude con la tesi della subordinazione della divinità rispetto all'astrazione ontologica dell'essenza (attributi dell'essere) - forse si dovrebbe connotare con categorie meno anacronistiche il discorso della dea parmenidea, la quale 'dimostra' la verità e non solo rivela e al contempo dimostra l'infondatezza delle tesi opposte; del resto la passività dell'ascoltatore dovrebbe essere interpretata piuttosto come 'oggettività' e ugualmente la subordinazione del divino dovrebbe valutarsi piuttosto come 'de-teologizzazione' nel senso che il principio o la fonte del vero non è più il divino mitologico in sé, ma l'ontologico cui il divino, del resto al pari dell'antropologico, è subordinato, è adeguato già per se stesso o si mostra per quello che veramente è, cioè di "non essere", se solo l'essere può e deve essere o almeno sono nella misura in cui il loro 'presentarsi' è compatibile con tale verità assoluta. Si consideri la connotazione 'soteriologica' della verità pitagorica, determinata dall'esigenza ineludibile della memoria e di una consequenziale identità celeste, rispetto a quella di aderenza alla realtà dei fatti in M. Tortorelli-Ghidini-G. Scalera McClintock, "Aletheia nel pensiero orfico", Filosofia e Teologia 4, 1990 73-84, 73-77. Tali configurazioni o istanze rivelative anamnestiche, come svelamento nella memoria di un'origine assoluta, suscitato dal bisogno soteriologico, non sono presenti in Parmenide che si distanzia da una simile fenomenologia religiosa e antropologia mitica. Per questo motivo poco valgono a svelare l' "enigmatico" Parmenide le recenti analisi di U. Di Toro che onnicomprensivamente legge nel poema solo una "maschera" metaforica secondo lo stile epico-misterico - cf. L'enigma Parmenide. Poesia e filosofia nel proemio, Aracne, Roma 2010 e qui basti la critica che si può facilmente avanzare che Parmenide non asserisce mai di voler velare sotto una trama di simboli e metafore per motivi comunicativi verso i suoi concittadini la sua verità, cioè "il retto discernimento delle cose della realtà" $(i b ., 160)$ e pervenire così alla metodologia che dispone alla vera conoscenza $(i b ., 167)$. Nemmeno possiamo intrattenerci debitamente sullo studio di Lisa A. Wilkinson che rivaluta giustamente la natura filosofica del proemio - cf. Parmenides and to Eon, Reconsidering Mythos and Logos, Continuum International Publishing Group, London-New York 2009, 70-79 - ma che acriticamente ritiene secondo la communis opinio che Parmenide abbia presente un pubblico o un qualche uditorio come destinatario e questo addirittura dovrebbe essere un perfetto esperto di Omero (pg. 74). L'unico destinatario della verità è esclusivamente il giovane cui si rivolge la dea e perché non venga anch'egli 'contaminato' o 'soggiogato' dalla falsità doxastica dei mortali (B8,60-61) occorre dopo l'esposizione della verità inconfutabile anche l'apparenza 'gnomica' dei mortali di cui ovviamente il giovane non può non avere qualche notizia e magari già con una sorta di istinto razionale avvertirla insoddisfacente ma che ancora non conosce compiutamente secondo la verità.

5 Si è tentato di spiegare l'originalità del poema parmenideo e il suo pensiero come "eccentricità" sfuggente a qualsiasi paradigma assimilabile con altri pensatori arcaici - cf. D. Zucchello, "Parmenide e la tradizione del pensiero del pensiero greco arcaico (ovvero della sua eccentricità)" in Studi di filosofia antica in onore di Livio Rossetti, a cura di S. Giombini e F. Marcacci, Perugia 2011, 165-178. Tuttavia, una simile impostazione sembra più pertinente alla sensibilità moderna che antica poiché tracce di omologazioni tra l'opera di Parmenide e le opere di altri autori a lui coevi sono continue e sufficientemente attestate nella dossografia, sebbene giustamente gli si riconosca un tratto distintivo inconfondibile. Sembra però eccessiva e distonica una rottura culturale per cui Parmenide vorrebbe offrire di sé come "nova figura de autoridade" poiché "se Parmênides deseja aqui inaugurar um novo tipo de saber, o jovem que se coloca a caminho não poderia ser comparado ao poeta ou ao adivinho. Esse jovem tem uma outra atitude, parece não aguardar a vontade das Musas para ter acesso àquilo que deseja", come si riscontra in Cristiane A. de Azevedo, "Parmênides e o novo "mestre da verdade", Anais de 
indicata, a veder bene, ad eccezione della 'giovinezza' e del suo essere compagno

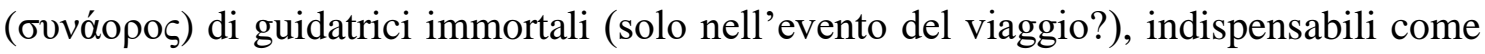
le cavalle per svolgere il viaggio stesso con il proposito di raggiungere la casa della dea. Sembra del tutto inverosimile che comunque non si possa arguire da ciò l'assenza di qualsiasi contatto con forme culturali e dottrinali o di un qualche apprendimento di esse, che del resto è dossograficamente accennato (in modo esplicito con riferimento al pitagorismo tramite il discepolato tenuto sotto Aminia e con lo stesso eleatismo originario tramite il discepolato aggiuntivo o esclusivo tenuto sotto Senofane di Colofone) benché a vario titolo diffidate. ${ }^{6}$ D'altronde, al di là delle loro declinazioni e individuazioni, le $\delta$ ó $\xi \alpha$ umane sono esplicitamente implicate nella composizione del poema a partire dal proemio e ne determinano in qualche modo l'andamento e il

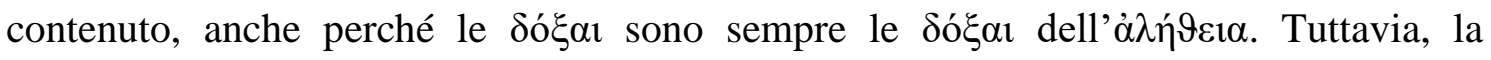
trasfigurazione 'epico-narrativa' impone un apprendimento totale ( $\dot{\alpha} \lambda \eta \dot{\vartheta} \varepsilon 1 \alpha / \delta$ ó $\xi \alpha \imath)$ per un 'discente' 'incontaminato' o 'aprioristicamente' scevro e non orientato rispetto a ogni potenziale 'alfabetizzazione' dottrinale, al di là di ogni spaccato storico-biografico.

La formazione culturale di primo ordine e di alti livelli che Parmenide mostra nella sua opera (ovviamente non materia insegnata dalla dea), accerta che egli conosca le tecniche retoriche e letterarie più elaborate. Pertanto non si può non sostenere che il proemio sia imbastito su una significativa dose di procedimento allegorico dimostrando al contempo una corrispondenza allegorica tra l'ordine simbolico e quello reale in termini poetici. La distinzione è tra l'allegorema (l'immagine dell'essere trasportato) e l'allegorizzato (il processo-percorso gnoseologico) ${ }^{7}$, visto che non è rilevabile nessun

Filosofia Clássica 9, n ${ }^{\circ}$ 17, 2015, 61-74, 70. La verità va solo esposta o raccontata e nessuno ne può essere maestro ma soltanto conoscitore se vi si predispone nel modo debito, secondo $\theta \dot{\mu} \mu 1 \varsigma$

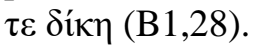

${ }^{6}$ Non si può affrontare minimamente un simile argomento in tale sede. Il linguaggio stesso e la forma esametrica impiegati nel proemio o nel poema suppongono necessariamente una conoscenza 'alfabetizzata' avanzata, non 'pre-scolare' o meramente 'rudimentale' e tanto meno 'popolare', per così dire. La stessa capacità di scrivere con tecniche 'poetiche' complesse induce a ritenere con certezza una qualche profondo discepolato, senza poi pensare ai contenuti che esse inevitabilmente comportano. Altro problema concerne la misura dell'influenza che dottrine pre-parminidee abbiano potuto esercitare sulla concezione di Parmenide. Non si può non decifrare dallo stesso proemio, ma anche lungo i frammenti, che le $\delta$ ó $\xi \alpha$, al di là delle declinazioni fino allora reperibili ed effettivamente reperite da Parmenide, hanno una funzione fondamentale e sono materia imprescindibile del $\mu \tilde{v} \theta$ o $\varsigma$ critico-elenchico della dea, anzi in qualche modo ne sono il movente più urgente o uno dei più urgenti.

${ }^{7}$ Pare davvero inverosimile che a Parmenide non siano giunte informazioni sulla metodologia allegorica dell'esegesi omerica condotta da Teagene di Reggio (v. aa. vv., Allegoristi dell'età classica. Opere e frammenti, Milano 2007), e che quindi impieghi un'immagine di questo 
evidente elemento testuale che spinga a ipotizzare anche per Parmenide la tradizionale separazione tra l'empirico e il concettuale con la conseguente individuazione di una duplice facoltà gnoseologica, quella sensibile e quella intellettiva ${ }^{8}$, limite rilevato nella

genere per significare poeticamente un evento essenziale, quello dell'incontro con la verità, connotato da una certa 'fatalità' singolare in modo da garantire la prospettiva di una sua storicità (essenziale) non in contraddizione con la sua 'visione' allegorica e soprattutto aperta a un'universalizzazione nel merito. Questo rilievo attenua o forse annulla del tutto le eccessive incertezze anti-allegoristiche avanzate da Patricia Curd la quale asserisce: "The evidence for the sources of this [allegorical] aspect of Parmenides' thought and for other early use of allegory is just too meager for certainty" in The Legacy of Parmenides. Eleatic Monism and Later Presocratic Thought, Las Vegas 2004, 19. Sicuramente l'allegoria non spiega tutto, ma vale soprattutto come filtro comunicativo di primaria efficacia per la composizione del proemio.

${ }^{8}$ Sono troppo 'razionalistiche' e fondate su una congettura alquanto drastica tra logos e mythos le suggestioni di G. Pugliese Carrattelli circa una visione 'strumentalistico-stilistico-letteraria' di Parmenide, che si sarebbe mosso in tal senso: "L'invocazione di figure antiche e famose [...] crea l'atmosfera conveniente a una $\alpha \dot{\pi} 0 \kappa \alpha ́ \lambda v \psi 1 \varsigma$; è evidente che il filosofo deliberatamente si avvale di forme del linguaggio mitologico, non perché, come pure è stato sostenuto, non gli si offrissero ancora efficaci strumenti linguistici per chiarire il suo pensiero, ma per procurare una disposizione e un'attesa quali, nel suo tempo e nel suo mondo, il nuovo e ardito messaggio esigeva. Entro questa cornice poetica si inserisce un discorso che non ha più bisogno di simboli

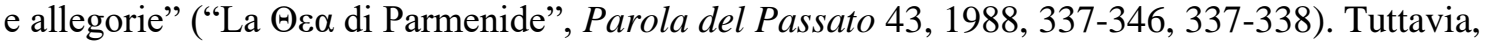
non si può non accennare almeno alle tre generiche tendenze denominabili rispettivamente come allegorica (basata su un orientamento razionalistico), mitico/storico-culturale (basata su un orientamento spiritualistico), minimale/'dismissiva' (basata su un orientamento misto), anche se si trovano reciproche combinazioni più o meno accentuate. La più antica è quell'allegorica concepita soprattutto da Sesto Empirico, che ne rappresenta anche la sua unica fonte, ma non disdegna anche di integrare elementi metaforici soprattutto platonizzanti (direttamente ricavati dal topos del carro viaggiante nel Fedro di Platone), ingiustificabili sostanzialmente per la dicotomia empirico/noetica delle facoltà umane. Nei commentatori moderni emerge la linea della "allegoria dell'illuminazione"; cf. in proposito ad es. C. M. Bowra, "The Proem of Parmenides.", Classical Philology 32.2, 1937, 97-112, passim; Panagiotis Thanassas, Parmenides, Cosmos, and Being: A Philosophical Interpretation. Vol. 57: Marquette Univ. Pr, 2007, passim; Nestor-Luis Cordero, By Being, It Is: The Thesis of Parmenides. Parmenides Publishing 2004. La linea metaforica si avvale dei contesti mistici-culturali e storici, soprattutto secondo ascendenze orfiche concernenti immagini di anabasi e catabasi, il cui primo interprete fu Diels-Kranz, e sovente il quadro icastico parmenideo viene ricollegato con lo sciamanismo. Cf. anche J. Henn Martin, Parmenides of Elea. A Verse Translation with Interpretative Essays and Commentary to the Text, Westport-Connecticut-London 2003, 1-10, in cui rievocando la figura del 'mediatore' vate-aedo sciamanico tra il divino e l'umano come descritta da Mourelatos (op. cit., 42-43) ne enfatizza la competenza metrico-musicale parmenidea a base di tricolon, di cui offre una tabella statistica comparativa con Omero e Esiodo, competenza giudicata quasi del tutto eguagliabile alla formularità omerica contro l'aspro giudizio esteticoletterario che Proclo ha rivolto ai versi parmenidei. Si arriva a dire: "This statistic would suggest that Parmenides composed his verses with slightly more attention to musical phrasing, rhetorical effect, and dramatic flourish, the confluence of which can only be produced with great deliberation and care" (ib., 5). Tale connessione è stata ribadita in Stanley Lombardo, Parmenides and Empedocles. Grey Fox Press 1982, ma criticata nella sua possibilità storica nella Grecia antica in G. S. Kirk-J. Raven-Malcom Schofield, The Presocratic Philosophers: A Critical History with a Selection of Texts. 2nd ed. Cambridge: Cambridge University Press, 2007. L'orfismo è stato sostenuto invece da John A. Palmer, Parmenides and Presocratic Philosophy. Oxford University Press, 2009. Le giuste obiezioni contro tali associazioni e le sue 
descrizione anacronistica di Sesto, che però non può essere scartata tout court. Questa specie di sovrastruttura letteraria non deve essere intesa come soppressione dell'elemento storico-biografico unico (e in se stesso irripetibile), ma come significato ulteriore e integrativo, in qualche modo immanente di quanto realmente $\mathrm{e}$ individualmente nonché, verrebbe da dire, 'fisicamente' avvenuto che comunque nell'essere trascritto ha comportato inevitabilmente una certa trasposizione semantica (non puramente da ไ̌ $\sigma \omega \rho$ alla maniera erodotea, in questo caso di autobiografismo comune). La detta trasposizione acquisisce pertanto almeno una nota di tipo paradigmatico e quindi ovunque e per sempre valida, ossia trascendente la mera esperienza personale, anche perché il suo contenuto è assolutamente universale, anche se incarnato nel viaggiatore verso la casa della dea e nell'ascoltatore delle sue parole. Questo contenuto è noeticamente disponibile appunto nella sua essenza senza più tratti di ambientazione di quell'unicum che devono ugualmente persistere come segnale di una circostanza di garanzia ed esplicativa, all'incirca nella modalità in cui funzionano le cornici introduttive nei dialoghi platonici, anche al fine di non far perdere il contenuto allegorico quale espediente isagogico al messaggio vero e proprio che ne segue all'interno del corpus della composizione. Ne consegue che chiunque può (deve forse)

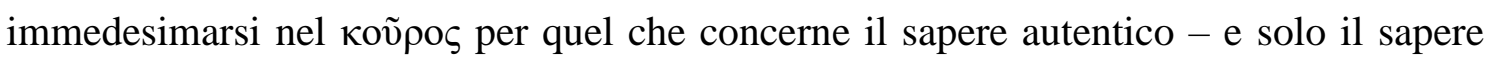
autentico può essere appunto sapere. A tale fine si presentano alcuni schizzi di ambientazione cosmologica alquanto decisivi e focalizzati con energica enfatizzazione su uno spazio sicuramente non ordinariamente accessibile, descritto in conformità a un punto di vista più mentale che fisico, anche se si segnalano note di trasporto fisico nel vero senso della parola, poiché nell'evento storico-biografico effettivo per giungere dove si è giunti è occorso oltrepassare alcune coordinate geo-cosmologiche.

debite decostruzioni soprattutto sul piano della ritualità di iniziazione orfica (poco conosciuta, per la verità) sono reperibile in Leonardo Tarán, Parmenides: a Text with Translation, Commentary, and Critical Essays, Princeton University Press 1965, che rappresenta una sorta di manifesto della tendenza 'dismissiva'. Questa tendenza si basa sull'insostenibile 'irrilevanza' filosofica del proemio e sul suo tono che si adatterebbe soprattutto a un'esperienza individuale pari a "spiritual awakening" (ib., 26-28; cf. anche Scott Austin, Parmenides: Being, Bounds, and Logic. Yale Univ Pr 1986). Cf. la quadripartizione delle interpretazioni "literales, religiosas, alegóricas y racionalistas" in Lambros Couloubaritsis, Mythe et philosophie chez Parménide, Bruxelles 1986, 80. 


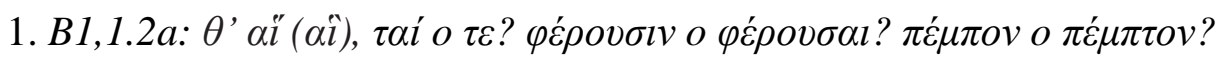

Il pronome relativo presenta il primo problema filologico ${ }^{9}$, poiché la versione $\theta$ '

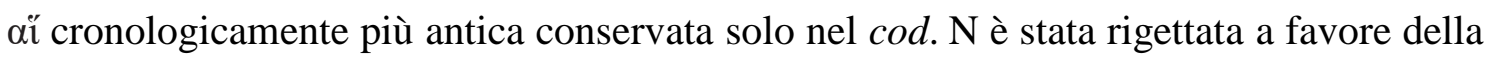
lezione $\tau \alpha$ í dei più recenti L ed $\mathrm{E}$ i cui testi sovente si riportano acriticamente nelle varie edizioni. ${ }^{10}$ Questo esito è dovuto al fatto che il $\operatorname{cod} . \mathrm{N}$ è ritenuto quello in cui si verificherebbero i maggiori errori, nonostante la sua unica assoluta anteriorità e coerenza interna. Che $\theta^{\prime}$ aí sia poziore rispetto a $\tau \alpha i$, è suffragato anche dalla variante $\tau \varepsilon$ dei codici $\varsigma$, i quali vantano una tradizione indipendente rispetto ai detti due codici posteriori $^{11}$, ma lasciano emergere un compromesso molto probabilmente posteriore per non avere compreso la valenza di $\theta^{\prime}$ aí. Di primo acchito, tale lezione non sembrerebbe genuina e magari sarebbe il frutto di un errore dittografico dell'omoteleuto nel successivo $\varphi \varepsilon ́ \rho o v \sigma \underline{\alpha \imath}$ (non ripetibile in B1,25, dato che per esso non è contemplata la variante $\varphi \varepsilon ́ \rho o v \sigma \alpha 1$, e non per altre ragioni poco verificate e controllate ${ }^{12}$; mentre è

${ }^{9}$ Laur. 85,19 sec. XIII; cf. A. Kochalsky, De Sexti Empirici adversus Logicos libri quaestiones criticae, Marburg 1911, 9-104.

${ }^{10}$ Risp. Laur. 85,11 sec. XV e Par. gr. 1964 sec. XV.

${ }^{11}$ Si tratta dei codici deteriores e recentiores: $\mathrm{A}=$ Paris. sec. XV; $\mathrm{B}=$ Berol. Phill. $1518 \mathrm{sec}$. XVI; R = Regimont. $16^{2} 12 \mathrm{sec} . \mathrm{XV}$; V = Ven. Marc. 262, sec. XV). Si potrebbe sospettare che $\tau \alpha$ í sia l'esito dell'enclitico $\tau$ ol, che sarebbe stato accentato per la successiva enclitica $\mu \varepsilon$, e in tal modo sarebbe stato difficilmente distinguerlo dal maschile toí stridente in qualche modo con ớ $\gamma o v \sigma \alpha l$ del verso successivo. L'ipotesi è allettante ma non vi sono a sostegno indizi sufficienti (soprattutto codicologici), del tutto smentita dall'attestazione di $\tau \varepsilon$ per niente impossibile e tanto meno di tono "ametrico", com'è stato inopinatamente bollato in Federico Condello, "Nuovi studi parmenidei tra filologia e dialettologia". [Rec. Passa 2009; Ferrari 2010], Eikasmos. Quaderni Bolognesi di Filologia Classica XXVII, 2016, 495-519, 502-503 .

${ }^{12}$ L'affascinante tesi di Enzo Passa ravviserebbe nelle varie identiche trascrizioni di $\vartheta$ ' aí del copista $\mathbf{N}$ in B1,24s (lezione accolta anche da altri eminenti studiosi, tra i quali: Cerri, op. cit., 148; Palmer, op. cit., 362; Coxon, op. cit., 53 addirittura con la variante d'interpunzione $\theta$ ', aí) tracce di una redazione psilotica recante un originario prezioso $\tau$ ' aĩ, come si annota in Parmenide. Tradizione del testo e questioni di lingua. Quaderni di seminari romani di cultura greca, Roma 2009, 57-59. Questa lezione non è del tutto priva di supporto testuale perché sostenuta da Claude Soumaise (Claudius Salmasius) stando a quanto si cita in Sexti Empirici Opera graece et latine, a cura di Alberto Fabricius, Lipsia 1718, 393 nota X). L'analisi di Passa sarebbe screditata proprio da B1,1 in cui un originario $\tau$ ' aĩ non potrebbe essere sostenuto non poiché in questo caso "il copista di $\mathrm{N}$ commette esattamente lo stesso errore proprio al v. 1, dove la coordinazione è del tutto impossibile" (Condello, op. cit., 503). En passant nell'apparato critico ad loc. in H. Mutschmann: Sexti Empirici Opera. II, Lipsiae 1914, 27 si

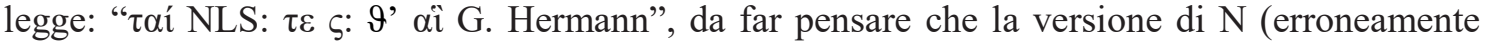
citato tra i manoscritti recanti la variante $\tau \alpha$ í) sarebbe stata avallata anche dal filologo tedesco. Si noti l'accento acuto in B1,1.24 in modo da rendere ambigua la morfologia del lemma, se si debba trattare, cioè, di pronome relativo o di articolo o di particella (equivalente a ci), per la enclitica che segue $(\mu \varepsilon \circ \sigma \varepsilon)$, situazione non infrequente in epica e invece evitata nel parallelo 
B1,13 in cui l'accento grave di $\theta$ ' aì esclude l'accezione di articolo e non di particella, per la quale esiste anche la forma tonica. Negli altri due casi potrebbe essere un articolo per questa medesima ragione, ovvero quella di acquisire e non di mantenere l'accento acuto per l'enclitica che segue. L'obiezione di Condello sarebbe valida se la funzione sintattico-semantica di $\tau \varepsilon$, oltre all'appunto metrico di per sé irricevibile che non rende indispensabile una forma di pronome relativa nel modo in cui si legge dalle rispettive varianti da considerarsi correzioni, fosse limitata a quella coordinativa. Questa funzione non sarebbe strettamente necessaria nemmeno nel parallelo in B1,24s, senza evidenziare che non è assolutamente necessario ritenere

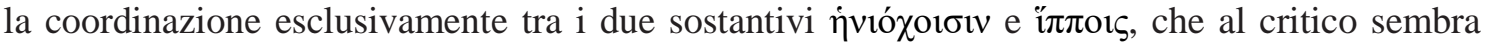
inadeguata per quanto concerne le ï $\pi \pi$ ot. Più nitidamente essa è da inserire tra le due intere

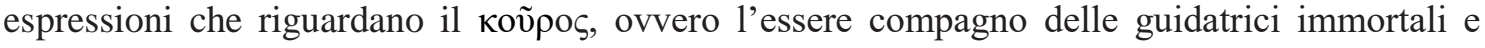
l'essere giunto con le cavalle che lo portano alla dimora della dea. Specialmente in epica, la funzione di tale particella può assumere una sfumatura di generalizzazione di qualche condizione permanente o di un'abitudine o di significare ciò che è ben conosciuto (e il fenomeno dell'essere trainati da equini speciali è un topos variamente attestato e in molteplici luoghi), funzione assimilabile a quella di $\tau$ or (cf. Od. II 276-7 e Il. XIII 115 con Il. XV 203). Farebbe problema la posizione perché la particella in genere segue il pronome e non lo procede. Potrebbe essere risolto avocando istanze metriche come 1" "ametrico" (?) $\tau \varepsilon$ dei codd. $\varsigma$ evocherebbe a detta dello studioso. Del resto non è stato mai considerato che un altro $\tau \varepsilon$ è presente nel primo verso e questa volta segue l'avverbio ő $\sigma o v$ subordinante, dal tono formulare nei poemi omerici. Condello continua a reputare la trascrizione del copista $\mathrm{N}$ in entrambi i paralleli citati un errore dovuto addirittura a un difetto neurofisiologico, a "un suo peculiare tic

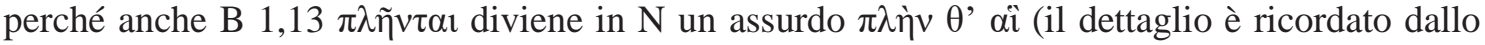
stesso P. [sc. Passa], p. 59, che tuttavia non ne trae conseguenze)". Per quanto il copista possa ritenersi gravemente e inguaribilmente affetto da una simile patologia, non pare realistico pensare che quasi meccanicamente ripeta lo stesso errore. Del resto non si vede perché l'errore

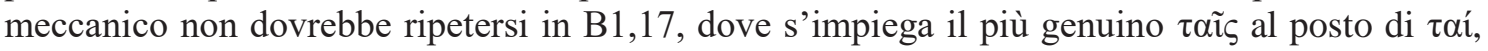
lezione appartenente a tutti gli altri manoscritti. Se proprio si deve parlare di errore o svista per quanto concerne B1,25, si dovrebbe menzionare l'omissione del finale $\varsigma$ da parte del copista di $\mathrm{N}$ nel trascrivere il vocabolo î $\pi \pi \mathrm{o}$, lezione condivisa anche dal copista di L. Non dovrebbe essere proprio peregrino ipotizzare che le ï $\pi \pi \mathrm{o}$ nella loro generica e nota funzione peculiare vengano indirettamente nominate in segno di riconoscenza per quanto hanno appena svolto in proposito, in modo da potersi tradurre: "e [le] cavalle ti portano. Rallegrati di giungere alla mia dimora [...]". L'indicativo presente è dal tono puramente narrativo e un'enfatizzazione dell'azione ancora in corso o continuata rispetto al momento in cui è iniziata rispetto al discorso diretto della dea. Del resto non si accenna al fatto che il portato scenda dal carro o altri movimenti di tal genere. Le due occorrenze di B1,1 e B1,25 rendono pensabile un $\tau \varepsilon$ anche per $\mathrm{B} 1,13$ per il quale però si sarebbe obliterata ogni traccia. La lezione $\pi \lambda \tilde{\eta} \nu \tau \alpha \iota$ (sia se il perfetto

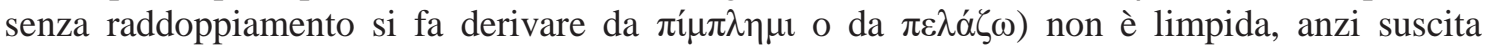
diverse perplessità, innanzitutto un perfetto in un contesto in cui si usa il tempo presente (B1,1112.14) e si sta descrivendo una struttura cosmica permanente nel modo in cui la si raffigura, come una sorta di parentesi incastonata nel racconto storico del viaggio. Del resto l'espressione suona come una nota aggiuntiva nel dettaglio della descrizione delle $\pi \dot{\lambda} \lambda \alpha 1$. Nell'accettare l'ipotesi che la lezione $\pi \lambda \dot{\nu} v \theta$ ' aî di $\mathrm{N}$ risulta da una improvvida correzione di $\tau \varepsilon$, che, tra l'altro, riserva la lezione $\alpha \tilde{\tau} \tau \alpha$ e non $\alpha$ voú, benché non si annoti in nessun apparato, si può supporre agevolmente una più che usuale ellissi della copula verbale: "e quelle del resto [sono] eteree [alte nell'etere piuttosto che di materia eterea] per i grandi battenti". Esempi omerici proprio con termini appartenenti alla gamma semantica dell'aggettivo $\mu \varepsilon_{\gamma} \alpha_{\varsigma}$ sono numerosi:

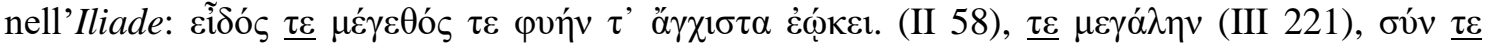

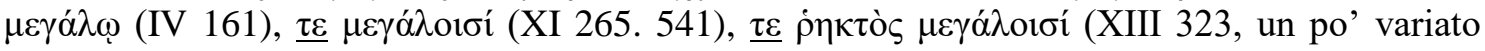

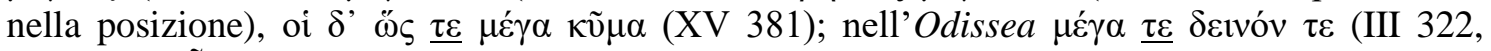

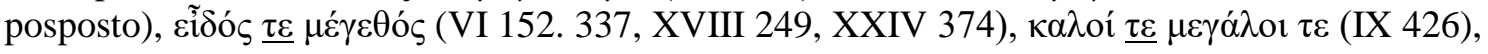

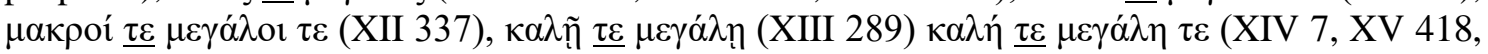

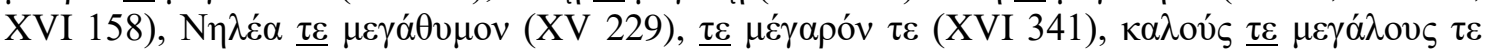




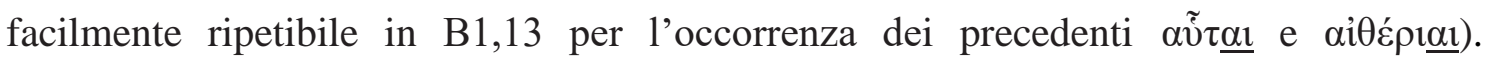
Tuttavia, il segmento $\theta^{\prime}$ del sintagma in esame prova che nel primo verso è presente la sequenza diffusissima in epica di due $\tau \varepsilon$ senza rapporto correlativo, poiché il secondo è connesso, come di frequente, con una particella di estensione.

Va rivelato che non si è mai analizzato il non insensato $\varphi \varepsilon ́ p o v \sigma \alpha \iota$ riportato da $\mathrm{N}$ al posto del $\varphi \varepsilon ́ p o v \sigma ı v$ presente in tutti gli altri, e metricamente formerebbe uno dei tanti casi di correptio epica. Filologicamente si sarebbe obbligati ad ammettere la variante $\varphi \varepsilon ́ \rho o v \sigma \alpha 1$. Ne dovrebbe conseguire che il verbo principale della proposizione relativa andrebbe ricercato in $\pi \varepsilon ́ \mu \pi \mathrm{ov}$, che non è attestato in modo assoluto ${ }^{13}$, oppure in una soluzione sintattica di ellissi. ${ }^{14}$ Questa interpretazione ellittica, per quanto addirittura corredabile di paralleli omerici, sembra poco plausibile e inutile. Invece $\varphi \varepsilon ́ \rho o v \sigma \alpha \iota$ è più accettabile come testo originale, anche perché filologicamente resistente a tentativi di

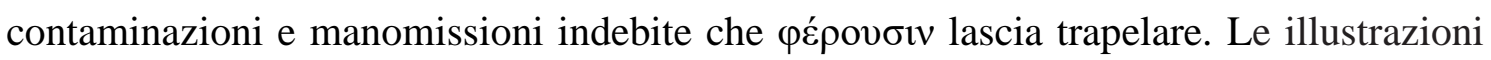
semantiche che il presente sarebbe indirizzato a definire generalizzazioni $o$

(XVIII 68). La lezione $\tau \varepsilon$, nonostante tutto, non convince e soprattutto lascia trapelare una manipolazione, oltre al fatto che non sussistono basi testuali per B1,13. Se, invece, di errore

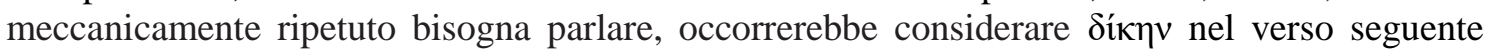
$(\mathrm{B} 1,14)$ comune a tutti i codici (dipendente da un errore presente già nell'archetipo e non quindi da un'anomalia motoria di uno scrivano) corretto in Sexti Empirici Opera quae extant [...], Typis ac Sumptis Petri \& Jacobi Chouët, Genève 1621, 217. Da autopsia sulla copia di $\mathbf{N}$ f. 124 riprodotta in Coxon, op. cit., 42 si deduce che si tratta di un caso di dittografia, fenomeno molto

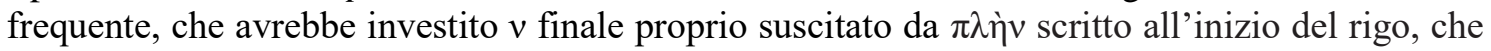

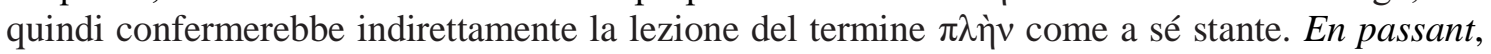
si dovrebbe ritenere più genuino l'accusativo per una sorta di attrazione (regressiva).

${ }^{13}$ L'unicità testuale di $\varphi \varepsilon ́ \rho o v \sigma \alpha l$ potrebbe corrispondere a uno dei tanti usi frequenti del

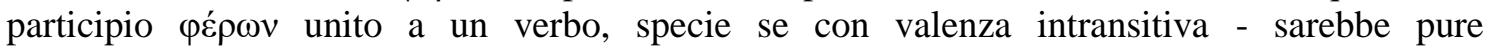
contestualmente più illuminante per l'implicazione abbastanza nitida di uno slancio irrefrenabile per quanto poco plausibile, rispetto a quella esplicativo-sussidaria molto ridondante. Si vedano alcuni esempi in Od. XX 146; XVII, 345; I 127. 139; Sofoc. Trachin. 622. Per tanto

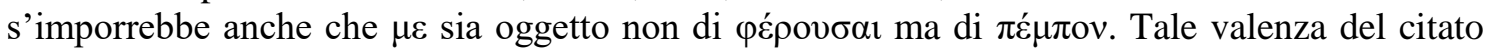
participio non solo illuminerebbe più intensamente la semantica e l'immagine poetica nonché concettuale che si sottintende, ma anche annullerebbe la possibilità che si possa scorgere una accezione proletticamente poco pertinente o pleonastica rispetto a quella nitida e enfatizzata in B1,4 e B1,25, oltre a rendere meno ripetitiva nel verso seguente l'espressione di ö́ non più impiegato nel proemio e sicuramente sinonimo di $\varphi \varepsilon ́ \rho \varepsilon \imath v$ ). Essa corrisponderebbe a una serie di tempi storici dei primi due versi, per cui l'indicativo presente risulta alquanto stridente.

${ }^{14}$ Casi omerici di costrutti ellittici di proposizioni principali addirittura con il lemma ì $\pi$ oı (Il. II

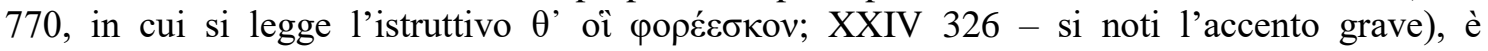
abbastanza strana in inizio assoluto di poema. Sicuramente questi casi sono inseriti in un contesto non di esordio di un componimento anche se funzionante sempre in inizio di verso, ma poco o nulla importa un tale aspetto. Nell' incipit parmenideo si offre un cotesto ben più intenso e narrativamente pregnante data la situazione di assoluta unicità e in qualche modo di inizialità, forse con qualche adombramento di iniziazione religiosa. 
attualizzazioni, non sono affatto decisive ${ }^{15}$; esse dovrebbero essere riservate esclusivamente al $\varphi \varepsilon ́ \rho \varepsilon ı$ del v. 3. Si tratta di evento storicamente circostanziato per un certo tempo di cui non è dato sapere nel dettaglio (non si tratta, infatti, di cronaca o di componimento epico-narrativo), certamente per uno scopo o oggetto gnoseologico preciso che si deluciderà man mano che si avanzerà nella scrittura. Del resto la denotazione di generalizzazione o di abitudine o meglio di costituzione è sufficientemente indicata dalla particella $\tau \varepsilon$ dei $\operatorname{codd}$. $\varsigma$, variante che, come si è già accennato, non sarebbe del tutto illogica e non è nemmeno metricamente impensabile, anzi tutt'altro. ${ }^{16}$ D'altronde, soprattutto l'impiego di $\pi \varepsilon ́ \mu \pi o v$ sembra pleonastico e marginale, poiché la sua funzione semantica può essere assolta da $\varphi \varepsilon ́ \rho o v \sigma \alpha$.

${ }^{15} \mathrm{Cf}$. ad es. Cerri che asserisce: "il tempo presente di questa relativa ( $\left.\varphi \dot{\rho} \rho o v \sigma \imath v\right)$ ne denuncia chiaramente la natura di definizione delle 'cavalle' in oggetto: sono 'le cavalle che mi portano fin dove l'animo giunge' (giunge?), cioè "le cavalle del pensiero" [...]. Il v. 1 fornisce così all'uditorio e al pubblico dei futuri lettori una spiegazione preventiva della funzione metaforica delle cavalle e del carro" (op. cit., 166). A parte che al limite questa funzione metaforica (e pare eccessivo che si tratta solo di questo connotato) piuttosto è affidata alla preposizione secondaria citata posta nella seconda metà del verso, ma questa preoccupazione preventiva non pare affatto emergere nemmeno implicitamente. Inoltre una tale simbolizzazione sarebbe già sufficiente-

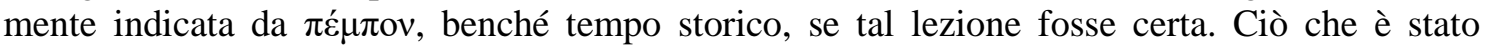
possibile nel passato potrebbe essere ripetuto. Aderente a tale giudizio si mostra Coxon che sostiene: "the present $\varphi \varepsilon ́ p o v \sigma ı v$ indicates that P. regards himself as still drawn by the mares, though the journey to the goddess is past. They must then symbolise his own impulse to philosophise (see Introd. Sect. 3 (iii)). Their significance is strongly emphasised both here and in 11.24-25" (op. cit., 270). Questa enfasi è una forzatura incongrua, dato che i presenti sarebbe in contesti e quindi con nuance differenti. La dea costata semplicemente al momento presente quanto descritto in $\mathrm{B} 1,1-5 \mathrm{ss}$.

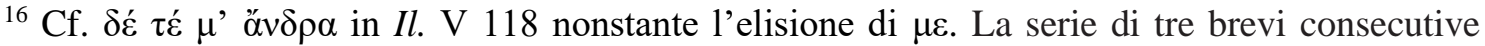

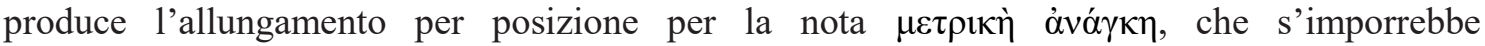
perfettamente anche per B1,25, ammettendo la più attendibile variante ï $\pi \pi \mathrm{ol}$ (al posto di ï $\pi \pi 01 \varsigma$ ), con una perfetta, benché rara, cesura trimemere e un'eftemimere, per enfatizzare magari con un

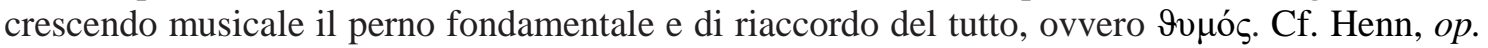
cit., 3 in cui si illustra il fenomeno ritmico-esametrico, vale a dire "the so-called rising threefolder, a verse consisting of three progressively lengthening metrical cola, whose caesura is bridged with an important word or epithet" e si continua con dettagli di tipo musicale con esempi comparativi. Oltre alle già citate maggioritarie occorrenze del segmento composto dell'enclitica $\tau \varepsilon$ con la sequenza derivante dalla gamma semantica concernente il tema $\mu \varepsilon \gamma$ nella qui precedente nota 12 , si registrano altre formazioni metriche simili. Cf. nell' Iliade:

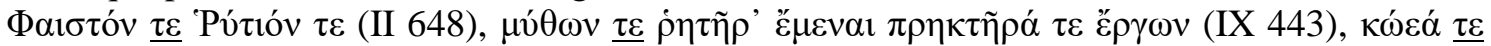

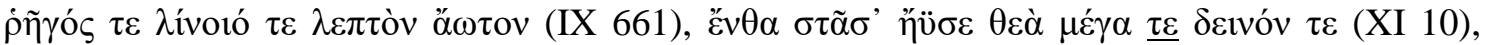

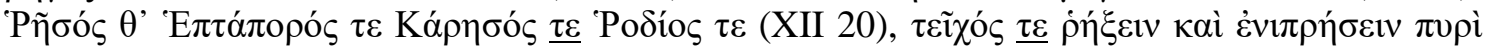

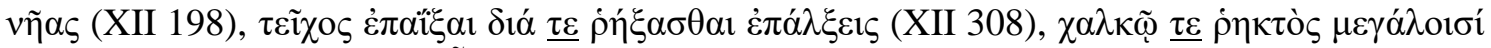

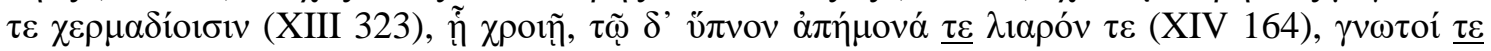

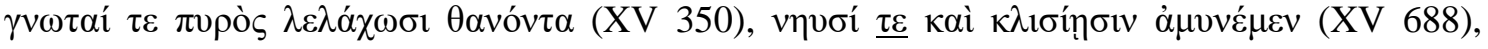

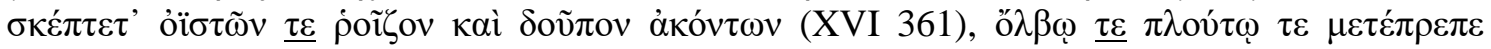

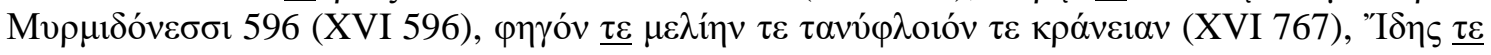

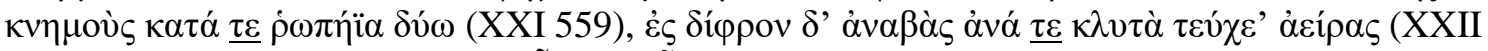

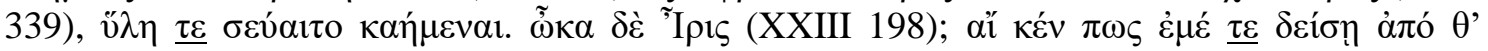


L'analisi si complica se si considera che i non irrilevanti codici NAB recano $\pi \varepsilon \dot{\varepsilon} \mu \pi \tau$ ov al posto di $\pi \varepsilon \dot{\varepsilon} \mu \pi \mathrm{ov}$. La lezione $\pi \dot{\varepsilon} \mu \pi \tau o v$ è stata universalmente ritenuta un errore o semplicemente ignorata. Se si considera la ramificazione manoscritta ricostruita, appare per lo meno curioso che un errore si verifichi in una prima derivazione dall'archetipo comune $\mathrm{G}$, rappresentata dal $\operatorname{cod}$. $\mathrm{N}$, per poi scomparire in un codice comune denominato $\mathrm{x}$, da cui si sono formati sia un codice $\mathrm{y}$ (originante a sua volta i codd. LE) sia un codice $\varsigma$, matrice dei cosiddetti codd. deteriores. Da un primo ramo di questi ultimi costituito dai codd. AB riaffiora $\pi \varepsilon \dot{\varepsilon} \mu \pi \tau o v$ mentre il secondo ramo costituito dai codd. VR conserva $\pi \varepsilon ́ \mu \pi$ ov (una contaminazione della corruzione in $\mathrm{N}$ e quindi in G?). Occorre evidenziare che la lezione ritenuta corrotta non è del tutto assurda, anzi forse quella più intellegibile. Infatti, $\pi \varepsilon ́ \mu \pi \tau o v$ può riferirsi a $\mu \varepsilon$ nel senso che Parmenide intenderebbe indicare il numero di chi è insieme al lui nel viaggio e quindi quello assegnato nell'ordine delle posizioni a partire delle anteriori, come quinto (meno plausibile l'accezione di quinto posto in forma di neutro avverbiale) - da scartare l'accezione di nome proprio, al pari di Quintus latino, perché non attestato in questa forma in greco. Si anticipa che Parmenide non è solo, anzi è il quinto di quanti intraprendono il viaggio che si va descrivendo, dopo o dietro le altre quattro presenze, costituite dalle già indicate cavalle e dalle giovani Eliadi che saranno svelate (il numero quanto potrebbe avere una connotazione anche simbolica, come i quattro punti cardinali, i quattro elementi basilari cosmici) - probabilmente corrispondente al medesimo numero implicito delle cavalle (il plurale sta in effetti per un duale per entrambi i soggetti, proprio perché la designazione di quinto per sé rinvia anche

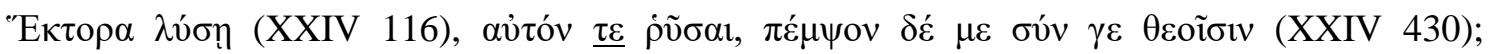

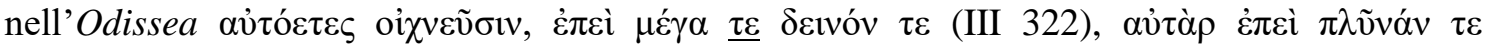

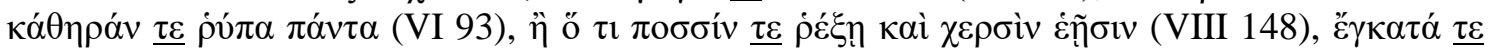

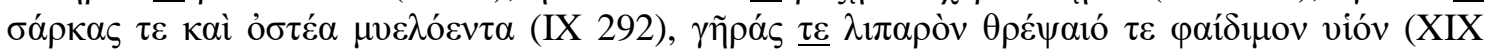

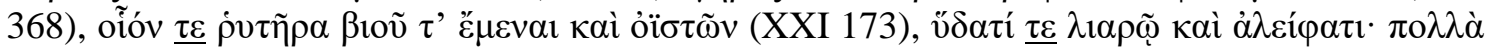

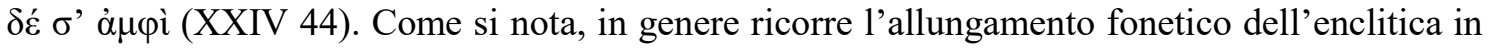
versi in cui è ripetuta e quasi sempre la prima occorrenza è sentita tale. Si osservi come la

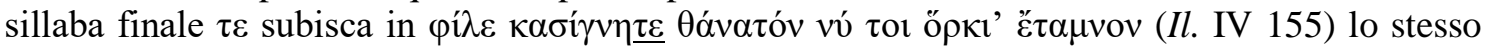
fenomeno metrico della particella negli altri versi citati. Inoltre l'enclitica $\tau \varepsilon$ dovrebbe ricevere l'accento acuto perché seguita dall'enclitica $\mu \varepsilon$ (risp. $\sigma \varepsilon$ nel parallelo successivo) in modo da agevolare la percezione prosodica dell'allungamento vocalico. A questo punto la relativa non sarebbe formulata per niente e il participio potrebbe quindi anche essere transitivo e avere come oggetto $\mu \varepsilon$, nel senso di "portandomi". Semanticamente ha una pregnanza più incisiva e meno

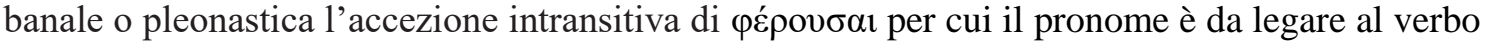
$\pi \varepsilon \dot{\varepsilon} \mu \pi \mathrm{ov}$ con iperbato, che del resto si ritrova anche nel verso seguente: "I $\pi \pi \mathrm{ol} \tau \dot{\varepsilon} \mu \varepsilon \varphi \varepsilon \dot{\rho} \rho 0 v \sigma \alpha 1$,

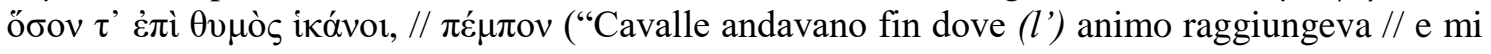
(ci) trascinavano"). 
indirettamente a pensare agli esseri equini e alle accompagnatrici immortali, numericamente distribuiti nella corrispondente coppia). Del resto, il plurale ï $\pi \pi 0 \imath$ non di rado indica una squadra equina da biga, che il numerale preciserebbe ulteriormente.

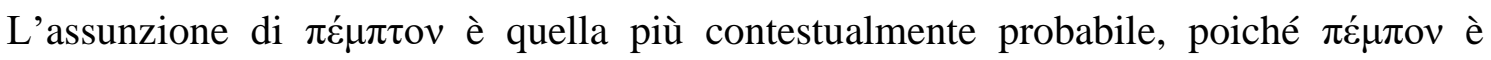
estremamente ridondante e anche poco confacente nel movimento di trasposto che si sta rievocando. ${ }^{17}$ Con la variante numerale si tratterebbe di un tocco stilitico-poetico non indifferente dotato di un'efficacia descrittiva di primo ordine: con un semplice numerale si sarebbe già preindicato con eleganza narrativa che non solo era soli come passeggero o viandante, e avrebbe creato una suspense circa altre presenze, oltre a riprodurre nel lettore quasi lo stesso effetto che l'evento ha suscitato nella percezione dell'autore. ${ }^{18}$

${ }^{17}$ Il verbo $\pi \varepsilon ́ \mu \pi \varepsilon v v$ indica piuttosto il senso di inviare, mandare, rimandare. Può senz'altro significare anche condurre, accompagnare, veicolare, ma in certi contesti alquanto circoscritti e in genere ascritti a esseri umani o divini: cf. Il I 390; Od. XIV 336; Sofoc. Trachin. 571 (centauro Nesso). L'epiteto ó $\pi \dot{\varepsilon} \mu \pi \omega v$ per antonomasia è riservato a Ermete (Sofoc. Filot. 133),

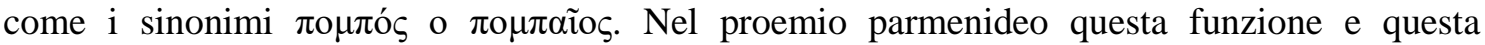
demarcazione di dirigenza nel viaggio sono da indirizzare piuttosto alle giovani $(\mathrm{B} 1,5)$ - per quanto le cavalle siano $\pi \circ \lambda \hat{\varphi} \varphi \rho \alpha \sigma \tau o$, per la loro funzione esse rimangono animali da traino di un carro come si specifica subito dopo da dovere essere guidate o condotte. Un altro motivo a sostegno dell'inautenticità di $\pi \varepsilon ́ \mu \pi o v$, è che il verbo non è mai impiegato in senso assoluto senza indicare il luogo in qualche modo determinato dove si è condotti, come nello stesso proemio l'occorrenza (a questo punto unica) indica chiaramente in B1,9.

${ }^{18}$ Cf. Il. XXIII 351; Od. IX 335; Thucid. I 46,3. 61,2; III 19,2; Platone Apol. 32c5; Fileb. 67a14 $(\pi \varepsilon ́ \mu \pi \tau o v=$ al quinto posto, come in Leg. 885a5). Cf. Caritone De Chaerea et Callirhoe I 9,2,3

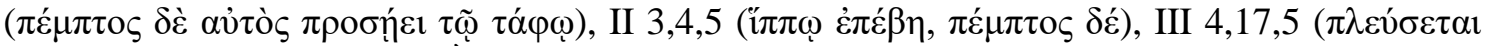

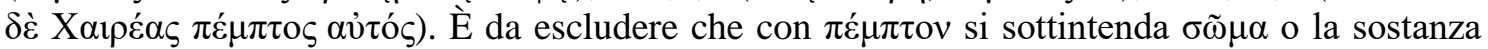
uranica (celestiale) o la cosiddetta quintessenza. A Pitagora è attribuita la dottrina cosmogenetica per l'azione del fuoco e del quinto elemento (H. Diels, Doxographi Graeci, BeroliniLipsiae 1929, II 6.2), ricordata da Olimpiodoro in contrapposizione al pitagorico Ippocrate di Chio (4,397.4ss. DK). Cf. Filolao B12 secondo una testimonianza di Teo di Smirne, in cui si

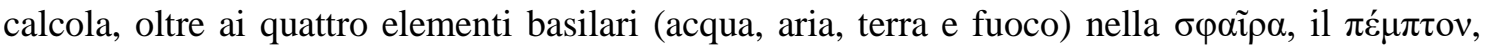
"quello della sfera attrattiva (che tira verso di sé, o centripeta?)" (ő $\tau \tilde{\alpha} \varsigma$ $\sigma \varphi \alpha i ́ p \alpha \varsigma$ ò $\lambda \kappa \alpha ́ \varsigma)$. Diels preferisce leggere ó $\lambda \kappa \alpha ́ \varsigma$ come un sostantivo e lo rende "Lastschiff" indicando però con il punto interrogativo una scelta non convinta anche come testo (segnale non tracciato nelle edizioni precedenti). In tal modo si recepisce non senza dubbio la critica Wilamowitz che giudica un'inserzione erronea ó $\lambda \kappa \alpha ́ \varsigma$ da parte di un amanuense che non avrebbe compreso ó $\lambda \kappa o ́ \varsigma$ reso con volumen, illustrato come qualcosa di tondo arrotolato o ammassato, come "Schlangenleib" o "Zunge" in Plato, Berlin' 1920, 91s, in cui si esclude categoricamente che possa riferirsi alla quintessenza platonico-aristotelica. Una simile valutazione indica comunque un movimento che si contrae in sé - comunque è difficile vederlo nel testo di Filolao, soprattutto perché si muta infondatamente la lezione. Non si dovrebbe esitare nell'interpretare ó $\lambda \kappa a ́ \varsigma \varsigma$ come aggettivo e assimilabile all'immagine d'immutabilità ( $\alpha \mu \varepsilon \tau \alpha \dot{\alpha} \beta \lambda \eta \tau o v)$ e della sua natura eterea ( $\alpha i \theta \hat{\varepsilon} \rho ı v)$ di tale elemento attribuita ad Aristotele nel senso che non si muove ma è principio di movimento degli altri elementi (cf. Diels, op. cit., I 3.22; II 7.5). Stobeo attribuisce a Parmenide la tesi uranica che il comune cielo, in effetti, sarebbe etere intorno all'estremo superiore rispetto al tutto (ib., II 7.1, 22s.). 
Allora il tempo principale andrebbe ricercato proprio in $\varphi \varepsilon \rho o ́ \mu \eta \nu$ del v. 4, che nasconderebbe anche un valore destinale in tale forma medio-passiva. ${ }^{19}$

Una simile opzione filologica e quindi traduttiva non pare comunque del tutto soddisfacente, anche se l'analisi sinottica e codicologica finora condotta ha messo in evidenza e provato in modo alquanto indubitabile che il cod. $\mathrm{N}$ è l'unico completamente affidabile e non compromesso con alterazioni spurie, considerata anche la cronologia. Pertanto non si può rinunciare alla lezione $\theta$ ' $\alpha$ î con l'inevitabile conseguenza sintattica che impone una disambiguazione morfologica di aí come articolo, fenomeno ripetuto in B1,14 ${ }^{20}$ (a differenza di B1,13 non necessariamente pronome relativo). ${ }^{21}$ Inoltre, se

${ }^{19} \mathrm{Si}$ potrebbe quindi approntare la seguente traduzione: Cavalle portandomi (Quando cavalle mi portavano), fin dove l'animo raggiungerebbe, per quinto (al quinto posto o meglio con gli altri quattro soggetti) [...], lì ero portato;". L'ottativo iאóvoı conserva la sua valenza originaria di potenzialità. Si evita così, anzi si annulla automaticamente proprio la speciosa diatriba esegetica vertente a individuare a quale verbo sarebbe assegnata la reggenza della secondaria in questione. Si rammenti la traduzione del Diels che coordina la proposizione subordinata alla relativa, opzione non maggioritaria accolta anche da traduzione di Giovanni Reale, che lo coordina con il verbo presente della proposizione relativa (v. Parmenide, Poema sulla natura, Milano ${ }^{22004,}$ 87) e coincidente con quella di Giovanni Cerri (Parmenide di Elea, Poema sulla natura, Milano 1999, 147), per limitarsi alle ultime proposte traduttive italiane. Si osservi la parafrasi davvero inammissibile di Richard D. McKirahan: "the mares which carry me as far as my spirit ever aspired were escorting me" (op. cit., 145). Tuttavia, questa distinzione per quanto legittima, risulta alquanto speciosa, poiché il verbo della principale $\pi \dot{\mu} \mu \pi \mathrm{ov}$ e quello della relativa

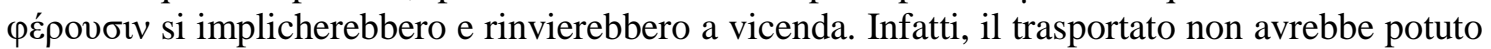
iniziare il suo viaggio se non fosse legato alle cavalle destinate a tale trasporto. Tali inutili

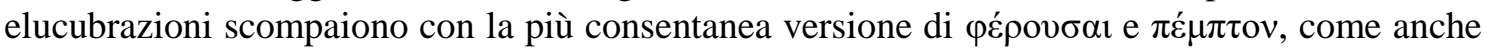
quelle che si sono profuse per comprendere il valore dell'ottativo in questione. Prevalgono due tesi interpretative in proposito, quella che lo giudicherebbe indicare una "indefinite frequency" come ad es. in Coxon, op. cit., 270, riecheggiato acriticamente da Bernabé con tali integrazioni che paiono peggiorative: "o optativo hikánoi indica aqui frequência indefinida, o que, na opção b1) poderia indicar a ideia de que é um caminho pelo qual marcha com frequência, entretanto, a opção b2) indicaria que as éguas o levam habitualmente por ali [quali ali?], onde ele quer, frente ao modo passivo em que e levado na ocasião extraordinária." (A che pro ripetere l'occasione straordinaria se essa ha già esaurito la sua funzione?), quella della possibilità come si legge innanzitutto in Mourelatos (op. cit., 17 nota 21), riecheggiato da Conche (op. cit., 44). Ma cosa dovrebbe significare la possibilità in tale contesto, se cioè il viaggio è stato già possibile, anzi perfettamente compiuto nel passato? Del resto, si cita giustamente la tesi di S. Furley che sottolinea l'unicità assoluta del viaggio (cf. "Notes on Parmenides", in E. N. Lee-A. P. D. Mourelatos-R. M. Rorty (eds.), "Exegesis and Argument: Studies in Greek philosophy presented to Gregory Vlastos", Phronesis 1973 (Supplementary Volume) 1, 1-15, 3 nota 5. Ne consegue che la destinazione del viaggio era quella che sarebbe stata raggiunta dall'animo, se non vi fossero ostacoli, che in qualche modo si lasciano trapelare nei due versi successivi, o per prevenire una tale eventualità funesta (in termini gnoseologici). Il tragitto fisico-cosmologico si è reso necessario per evitare eventuali insidie, anzi le insidie già presenti nel mondo umano, in tale processo di contatto gnoseologico-intellettivo.

${ }^{20}$ La costruzione sintattica nella forma di pronome relativo è rara ma non assente e si sono

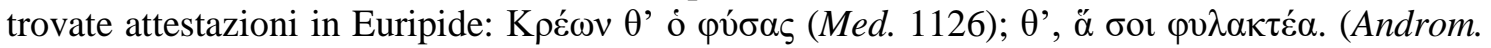

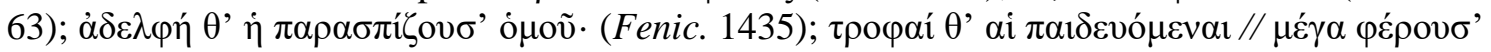

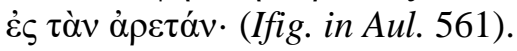


fosse da recepire come pronome relativo, non si troverebbe verbo finito a cui appoggiarsi, almeno che non ci si avvalesse di nuovo della forma ellittica della prima proposizione del proemio parmenideo. Il participio quindi è in forma congiunta mentre l'articolo in posizione anforica mette in risalto le cavalle e serve a specificare anche il loro genere femminile, essendo il sostantivo in greco appartenente anche al genere maschile (unico segnale nel contesto). ${ }^{22} \mathrm{Si}$ presenta quindi la traduzione nel modo seguente:

Cavalle, ed esse, portandomi [Quando cavalle, ed esse, mi portarono], fin dove animo raggiungerebbe, per quinto [con le quattro (presenti)], ....

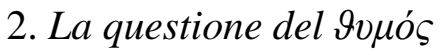

Di primo acchito, sembra che si possa segnalare un parallelismo ben delineato tra i nominativi del primo verso, "cavalle" e l' "animo" 23 , da far risaltare una

${ }^{21}$ Il fatto che per tale versione non sia rimasta traccia di una lezione con $\tau \varepsilon$, a differenza dei relativi paralleli nel proemio, è il segno poderoso che questa variante è posteriore anche a quella unanimemente accettata di $\tau \alpha$ í nei paralleli che quindi tutti riportano la versione secondaria

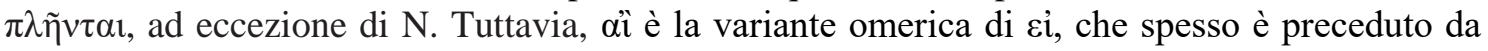
$\pi \lambda \eta v v$ in modo che il senso è chiarissimo, e cioè: "quelle [sc. porte] (sono) eteree tranne che ai grandi battenti" o nella struttura portante, ovvero se anche gli stipiti o l'intelaiatura fossero eterei non potrebbe nemmeno essere percepite le porte che di quelli sono composte, come in qualche modo si esplicita subito dopo in B1,16-20. Che siano alte nell'etere, è banale $\mathrm{e}$ pleonastico poiché già cosmologicamente significato e intuibile. Inoltre nell'etere è difficile infilare le chiavi, per quanto simbolica ne possa essere la descrizione. Se le porte non fossero sostanzialmente di materiale etereo, sarebbero di una pesantezza quasi impensabile per un luogo appunto sostanzialmente etereo, che invece la via stessa dovrebbe anche garantire come basamento.

${ }^{22}$ La variante di articolo è quella più plausibile, per cui si tratterebbe di un'enfatizzazione retorico-descrittiva sulle cavalle e sulla loro indispensabile funzione. La traduzione quindi più plausibile è semplicemente la seguente: "cavalle, ed esse, ti portano". Il nominativo ì $\pi \pi 0$ al posto del dativo elimina qualsiasi ambiguità sintattica con l'espressione precedente. Inoltre nemmeno l'aggettivo $\pi 0 \lambda u ́ \varphi \rho \alpha \sigma o t$ riferito a tale sostantivo in 1,4 serve in tal senso.

${ }^{23} \mathrm{Si}$ è scelto di tradurre con (il più generico?) animo il greco $\vartheta u \mu$ ó quale sede interiore della facoltà intellettiva e dell'intendimento. Poco rilevante è il parallelo sollevato dal Diels con $O d$.

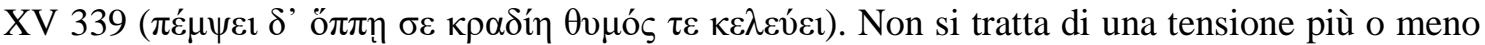
impulsiva e indeterminata in Parmenide, ma verso l'oggetto gnoseologicamente determinato o il punto dove è attingibile questo oggetto gnoseologicamente definito anche se ancora non noto in

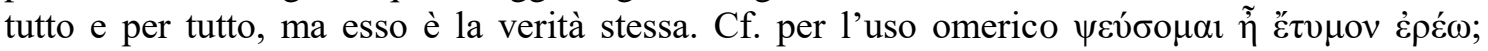

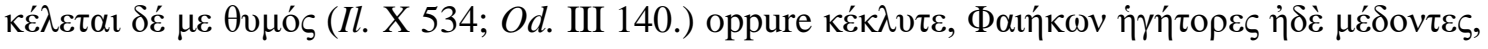

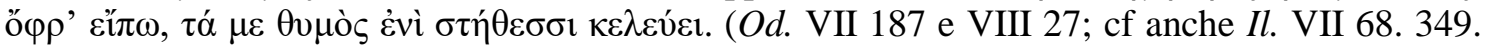
369, VIII 6, XIII 784; Od. VIII 204, XIV 517, XV 339, XVI 81, XVIII 352, XXI 198. 276. 342;

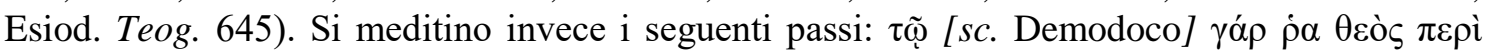

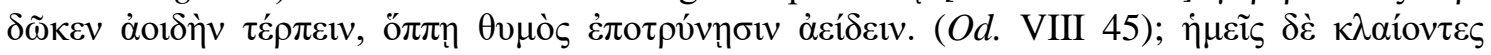


coordinazione intrinseca e intima tra i concetti espressi dai rispettivi verbi. L'azione delle prime è introdotta solo perché corrisponde a un potenziale fine del secondo. Proprio perché simile approccio dell'animo è in sé stesso potenziale per il narrante che rischia di non accedervi affatto al pari del resto del genere umano, si è resa necessaria una 'strumentazione' o 'organizzazione' di supporto esterno (adeguato e soprattutto per divina iniziativa e garanzia) per evitare un qualche fallimento alla naturale e umana inclinazione (intuitiva e intellettiva) di cui ovviamente anche Parmenide come tutti i mortali è dotato. La cornice storico-biografica indicativa e circostanziale di un episodio realmente e fisicamente (cosmologicamente) avvenuto, o comunque in tali termini si vuole che si comprenda (per prospettare anche una visione e topografia cosmologica), e pertanto indirettamente paradigmatizzabile in un quadro di allegorema dalla declinazione universale, ma anche esclusivo nella sua irripetibile unicità individuale, rappresenta ovviamente un qualche incipit assoluto in una certa concomitanza con la

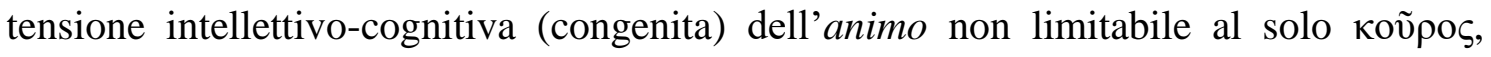
come è segnalato dall'ottativo iאávor, allusivo di una potenzialità teoricamente universale dal punto di vista antropologico. La subordinata, di cui íóvor è verbo, di tono esplicativo o dichiarativo, ovvero finalizzato a determinare il punto che l'animo avrebbe potuto raggiungere, si scoprirà essere la via che mette in contatto con la dea preposta allo 'svelamento' (non proprio rivelazione) della verità $(\mathrm{B} 1,26)$, ma via che potrebbe ugualmente anzi dovrebbe raggiungere qualsiasi animo. Tuttavia, in vista della sua riuscita infallibile si sono approntati espedienti specifici e mirati individualmente a

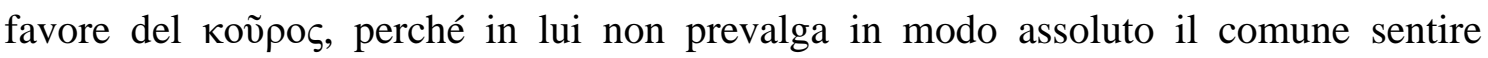
doxastico e quindi subisca la stessa sorte toccata ai mortali, di precludersi l'accesso alla verità. La costruzione sintattica del primo segmento proemiale in esame trova illuminazione nel confronto con B1,25. La dea ne accerta la natura illustrandone condizioni e modalità. Il giovane è potuto giungere alla dimora della dea solo perché le cavalle lo portavano sin lì ( $\varphi \varepsilon ́ \rho o v \sigma \alpha ı \mathrm{~B} 1,1)$, fin dove anche l'animo di suo avrebbe la facoltà di arrivare o toccare, proprio perché lo portavano ( $\varphi \varepsilon ́ \rho o v ~ B 1,4)$ sulla giusta e predestinata direzione verso il luogo in cui inizia il viaggio. Non si fa alcun accenno a una qualche eventuale descrizione del fatto che il giovane scenda dal carro o di qualche

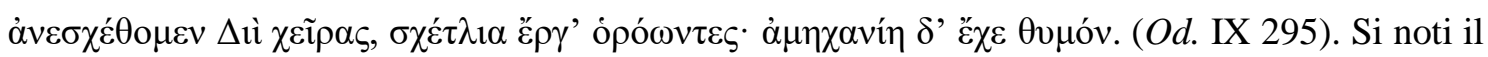

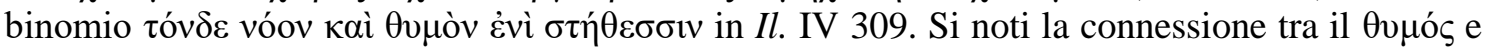

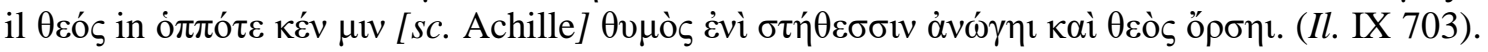

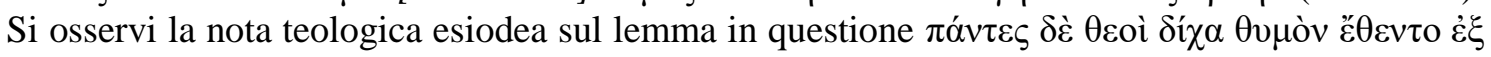
Éploos (fr. 204,95). 
suo diverso posizionamento che non sia appunto quello dell'essere portato dalle cavalle, funzione che appunto è in atto ancora al termine del viaggio, di cui il tragitto cosmologico è in qualche modo il preambolo 'narrativo' contenente accenti metaforizzanti. Se ne può arguire che tale funzione valga anche per il momento statico subito dopo l'arrivo alla casa della dea, dopo cui si rimane sul carro, e non solo vi si è adagiati per tutto il tratto in cui ci si è mossi con il traino del carro, almeno in base a quanto pervenuto ${ }^{24}$, situazione che vale implicitamente anche per le Eliadi. Quanto valutabile contestualmente come metaforico e simbolico non oscura l'evidente enfasi sul fatto che le cavalle sono presentate in qualche modo come animali esterni al loro portato e dotati di una certa autonomia di comprensione ( $\pi$ o $\lambda \hat{\varphi} \varphi \rho \alpha \sigma \tau o r$ B1,4), come se si trattasse di una 'armoniosa' sinergia intellettiva e in qualche modo intima con chi portano (per tutte le fasi del viaggio), benché sotto la direzione e la presidenza delle Eliadi. Infatti, quest'azione non può essere solo occasionale o storicamente condizionata o per qualche iniziativa personale, ma concerne soprattutto un interario mirante alla conoscenza piena e olistica, al contempo abilitante a scoprire in maniera unica e definitiva quanto i mortali hanno universalmente ignorato con la conseguenza di versare in uno stato (insipiente) di irreversibile smarrimento circa essa.

La denotazione di immagine mitico-simbolica generica relativa alla Sophia e al contenuto epico-sapienziale culturalmente condiviso, assegnato alle cavalle, per quanto innegabile (ma il giovane non dovrebbe essere stato preservato da tutto ciò al momento dell'inizio del viaggio, per il quale non ha altri mezzi o ausili se non le cavalle guidate dalle Eliadi, dato che non poteva non conoscere e intendere almeno il linguaggio impiegato per la sua trasmissione), è seducente ma non pienamente pertinente. L'elemento chiarificatore va ricercato nella proposizione relativa e soprattutto nel

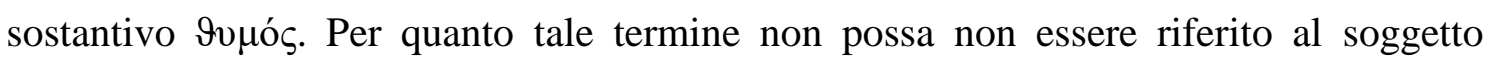

\footnotetext{
${ }^{24}$ È probabile che queste operazioni di accoglienza siano state descritte prima che la dea iniziasse il discorso vero e proprio del suo insegnamento (a partire dal fr. 2?), oppure siano date per scontate. Tuttavia la scena dell'arrivo e la ripetizione della formula delle cavalle che portano il giovane lasciano emergere che tale azione o trazione non sia solo fisicamente motoria o addirittura accessoria, come se si trattasse non di mere cavalle qualsiasi quelle che portano Parmenide, come anche si è accennato già nella nota che si è svolta sulla questione filologica della versione al nominativo (preferibile) piuttosto che al dativo del termine che le designa in B1,25. Logica vorrebbe che sia Parmenide, un vivente, sia le Eliadi, esseri divini, abbiano necessità del carro e delle cavalle per permanere nella casa della dea e magari non cadere nel vuoto. Ciò comporterebbe, ancora consequenzialmente, che il carro e le cavalle appartenessero intrinsecamente alle Eliadi, come strumenti e componenti della loro costituzione e funzione (mitica nell'ordine cosmico).
} 
narrante, ma in quanto antropologicamente costituito e appartenente al genere umano, estenderlo anche alle cavalle appare alquanto ridondante e tutt'al più marginale nel contesto, poiché va da sé. Non si tematizza anche per le cavalle per il semplice fatto che esse non devono accedere al regno della verità, di cui in qualche modo appaiono come 'figure' di garanzia e nel quale si dà a vedere che siano già situate, e per rendersene conto non possono non possedere il proprio $\theta u \mu$ ós. Le cavalle con le giovani immortali (che ugualmente non possono non possedere il proprio $\theta v \mu o ́ \varsigma$ ) si adoperano a traportare Parmenide per lo stesso fine, quello di entrare in contatto con la verità. ${ }^{25}$ Con la

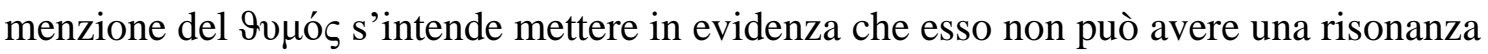
solo personale, ma deve assumersi come una valenza universale e di conseguenza antropologicamente strutturale e strutturante, cioè quale sede di quella capacità psicomentale o attinente alla sfera noetica comune a tutti (B16), benché anche teologicamente significante. Ciò che Parmenide è venuto a conoscere in questa forma personale, con il suo 'animo' non è altro che quanto ciascuno dei mortali potrebbe conoscere con il proprio, se solo ciascuno di costoro non si fosse lasciato deviare altrove

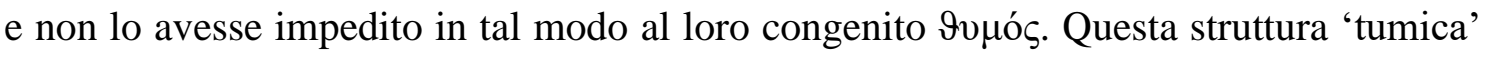
non può non appartenere anche alle cavalle, forse solo indirettamente. Si vuole illustrare

${ }^{25} \mathrm{Cf}$. sporadicamente si è avanzata l'ipotesi che il $\theta 0 \mu$ ó s si assegnasse esclusivamente alle cavalle; ad es. Jaap Mansfeld, Die Offenbarung des Parmenides und die menschliche Welt, Assen 1964, 231 in cui motiva in modo alquanto curioso "celui qui dépend complètement de l'escorte divine qui le conduit ne peut aller "aussi loin que va (son propre) désir". Non si dà a vedere che emerga questa totale dipendenza dalla scorta divina per il "compagno delle guidatrici immortali" (B1,24) magari solo di un'intesa profonda quasi simbiotica. Esprimono la stessa opinione sia Tarán in op. cit., 9s. che Fernando Santoro in Filósofos Épicos I : Parmênides e Xenófanes, fragmentos, Revisão Científica Néstor Cordero Rio de Janeiro 2011, 79. Tuttavia, la

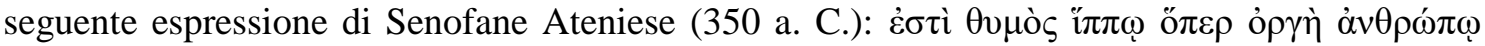
(De re equestri IX 2,1), farebbe sostenere che il $\theta 0 \mu$ ó s appartenga alle cavalle. Per accettare però l'attribuzione al giovane non è sufficiente la ragione che si legge in Coxon per un parallelo omerico (Od. XV 339) riscontrato dal Diels nel già citato Lehrgedichte, 46 scrivendo che pertanto Parmenide "alludes in the first instance to his own $\theta 0 \mu$ ó , of which the mares are the incarnation and symbol." (op. cit., 270). "In first instance" non significa un'esclusiva, inoltre "the incarnation and symbol" che le cavalle rappresenterebbero, non può essere solo immaginariamente descrittivo quasi a fare prevalere oltre ogni misura la simbolicità e rendere l'incarnazione quasi fittizia. Si tratta di una definizione prospettata propriamente per i personaggi in questione, ma il $\theta v \mu o ́ s$, al di là della sua semantica precisa, non può non indicare una qualità ben specifica che denota esseri intelligenti in genere e quindi una facoltà psiconoetica pertinente e la sua dinamica propria del tutto dispiegata. Si tratta di una disposizione connaturale e non di uno sforzo e tanto meno di un'impulsività momentanea e accidentale aneddotica come appare per l'eroe omerico (in cui tra l'altro il riferimento è esplicito). Ciò che non è lecito compiere è inserire nel tradotto un qualche aggettivo possessivo concordato con il sostantivo in questione, come sovente avviene a prescindere dall'opzione di riferirlo al giovane $o$ alle sue portatrici. 
che quanto si sta volgendo con il viaggio con le sue conseguenze noetiche, corrisponde

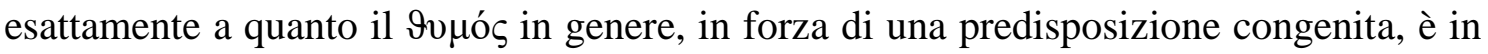
grado di raggiungere in sé stesso e per sé stesso, e riguardo alla sfera umana lo si può se in assenza di altri condizionamenti indebiti. La proposizione introdotta da દ̇ $\pi \varepsilon i ́$ (più causale che temporale) rappresenta l'inizio storico e al contempo la motivazione teoretica, per dir così, di quell'unicum che va dall'imbocco della strada alla casa della dea di cui si parla nei vv. 2-3. Si dovrebbe trattare di un fenomeno filosofico (o propedeuticamente filosofico) non pienamente assimilabile a quello (abbondantemente attestato) di 'rapimento' mistico o poetico per descrivere una 'verità' particolare basata su una rivelazione o ispirazione divina.

Il proemio fino a B1,24, cioè fino al momento in cui non compare il discorso diretto della dea, serve a inquadrare e collocare in una dimensione assolutamente trascendente e unica tutto il contenuto del poema, vale a dire proprio il discorso della dea nella sua interezza ${ }^{26}$, in una continuità universale con quanto si descrive con inevitabili accenti storici nei versi precedenti e ancor più nei primi cinque che ne rappresentano la narrazione incipiente, alla stregua di una compiutezza della situazione di chi sta narrando di aver saputo di essere stato condotto a imboccare la via giusta per la conoscenza. Benché si costruisca il riferimento con un materiale proveniente dalla topica mitico-poetica più o meno stratificata e stilizzata, nell'uso intelligente e calcolato delle rappresentazioni si può scorgere un cospicuo indizio di 'rottura', non contrassegnata da una polemica precipua e circoscritta a una determinata tematica o attitudine letteraria o culturale, che dir si voglia, né da una variatio avversativa rispetto a una qualche opinione particolare, come quella ad es. sull'arché presso i milesi. Il senso della 'rottura' non è poetico o sapienziale o religioso per contrastare una dottrina altra in parte o nel complesso. Si tratta invece di un impianto 'filosofico', inerente alla verità in sé stessa, nella sua appropriatezza noetica propria e olistica. Parmenide nel proemio si distanzia volutamente dai repertori tradizionali e stereotipati, giudicandoli in tal modo inadatti alla novità assoluta del suo pensiero, anzi alla noesis universale della verità in sé. Di conseguenza gli espedienti espressivi sono riformulati per focalizzarli sul rapporto 'unico' e al contempo 'complesso' tra il passato dell'evento del suo

\footnotetext{
${ }^{26}$ Purtroppo la tradizione dossografica, che ha conservato il proemio, non ha trasmesso nulla dell'epilogo del poema; ma si può supporre ragionevolmente che esso sia stato fatto coincidere con il termine del discorso stesso della dea, per cui non avrebbe presentato connotazioni di qualche valenza letteraria o filosofica strettamente rilevante a differenza del proemio.
} 
incontro con la verità e il suo presente che lo riattualizza non in forma reiterativa letteralmente ma letterariamente secondo i canoni e le trasposizioni anche metaforiche a lui disponibili nel comporre l'opera poetica. Si vuole rilevare che quell' 'essere stato accompagnato', che gli ha permesso di raggiungere la dimora della dea, è ormai passato, poiché ha avuto necessariamente un inizio temporale e biografico insieme a una sua naturale conclusione, ma la funzione gnoseologica che ciò ha rappresentato, e soprattutto i contenuti aletici che il discorso della dea veicolano, non possono non essere permanenti e trascendenti di fronte a quelle circostanze personali e in qualche modo contingenti. Infatti, per il proemio parmenideo non si tratta di raccontare fatti mitici o storici in modo epico o in conformità a innografie esiodee e quant'altro, ma di descrivere la condizione permanente, per così dire, 'formale/trascendentale', della conoscenza umana circa la verità, a mo' di una propedeutica metodologica, allo scopo di comporre una premessa di teoreticità assoluta, metastorica (divino-immortale), avulsa da espliciti interessi pedagogici, dottrinali o espressivi di qualsiasi genere.

Le cavalle "molto assennate" insieme al carro, oltre alla loro innegabile fisicità per quanto poeticamente funzionale, rappresentano la dimensione gnoseologica infallibile se guidata secondo la giusta direzione, però, si ribadisce, non di tipo mistico o esoterico o profetico, ma di tipo filosofico per cui si richiede un intervento altro, radicalmente incompatibile con la metodologia e le applicazioni finora eseguite ed esposte dai mortali. La condizione noetica antropologica trova il pieno sviluppo esclusivamente in chi con le proprie potenzialità noetiche, viene debitamente 'equipaggiato' in modo privilegiato perché prima (si potrebbe dire 'aprioristicamente') che esse possano esser coinvolte e magari irrimediabilmente dominate decettivamente da un arbitrio umano e quindi personale che comunque riproduca materialmente il contenuto desunto dal modo doxastico comune di presunto approccio al vero, fosse tale contenuto anche di natura teologica. Il giovane quindi viene 'strappato' o si lascia 'strappare' dall'eventualità rischiosa della comune condizione di ignoranza, di non percorrere nessuna vera via. La Dike garantisce che quanto ascolterà il privilegiato, è quanto gli uomini avrebbero dovuto sapere e invece ignorano per un'attitudine gnoseologica impropria, per aver ignorato decettivamente la via che permette il percorso della conoscenza. Si deduce che il filosofo intende porre immediatamente in risalto il percorso gnoseologico che stava per intraprendere con le sue facoltà noetiche o preposte alla funzione noematica secondo la via e lui, il giovane portato, quale singolare destinatario 'soccorso', riceve il 'singolare privilegio' di accedere alla verità infallibile. 
Pertanto si richiede l'unico percorso ancora ignoto e inesplorato $(\mathrm{B} 1,27)$ dalla mente umana in genere o meglio che la mente umana in genere ingannevolmente ha ignorato e quindi si è condannata a una deviante erranza (B6) in modo che non lo ha ancora esplorato come avrebbe dovuto e potuto, percorrendo una via che in effetti non esiste (se non nella mente stessa allucinata dei mortali che l'hanno percorsa e continuano rovinosamente a percorrere) o è da escludere dalla ricerca (B2,5ss; B6,3ss; $\mathrm{B} 7 ; \mathrm{B} 8,17)$. Questa devianza o erranza dipende ovviamente dal fatto che non ci si è aperti alla verità nella sua assolutezza inconfondibile, si direbbe oggi, 'oggettivamente', ma ci si è basati sulle 'connaturali' apparenze, si direbbe oggi, 'soggettivamente', secondo quanto confusamente si crede di percepire (B8,53ss). Proprio perché la metodologia epistemica umana è ormai compromessa e contaminata dall'errore e non fondata sulla persuasività inconfutabile, si sono attivati un apporto e un supporto di natura radicalmente diversa, divina, cioè inerente a una fonte altra incontestabile (ma comunque non incompatibile anzi logica, che si atteggia con la stessa procedura del ricercare umano) dello svelamento della verità senza pretese messianico-soteriologiche di tipo religioso. La sua accoglienza presso i mortali non è prevista almeno esplicitamente, anzi sembra piuttosto esclusa poiché non conservano quella capacità 'consona' che invece non è (del tutto) ancora compromessa nel giovane che così 'da vivo' e prima di essere irreparabilmente contaminato anch'egli, può essere partecipe della immortalità delle sue guidatrici delle quali è dichiarato 'compagno'.

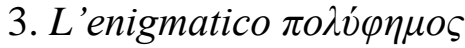

La unanime e unica possibile attribuzione di $\pi 0 \lambda u ́ \varphi \eta \mu o \varsigma$ ó $\delta \varsigma^{27}$ non è stata

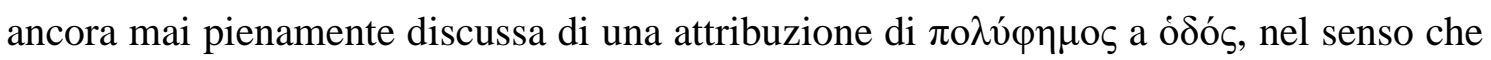

\footnotetext{
${ }^{27}$ Rapidamente è escluso che si tratti di nome proprio come il Ciclope nell'Odissea o Polifemo tra gli Argonauti o ancora il "divino Polifemo" compagno di Teseo e del giovane Agamennone secondo la menzione omerica in $\mathrm{Il}$. I 264 (cf. anche il personaggio mitico come raccontato in Pseudo-Apollodor. Biblioth. I 117, 4 e Antonino Liberale Metamorphoseon synagoge XXVI 5, 3). Una simile opzione dovrebbe basarsi sull'inaccettabile sospetto che il nome Parmenide fosse coniato più o meno simbolicamente da Platone nell'omonimo dialogo coniugando $\pi \alpha \rho(\alpha) \mu \varepsilon v(\varepsilon)$ '́ $\delta \eta$ con il significato di 'rimani-presso-l'idee' (colui che è fedele alle idee o dimora presso le idee). Platone è anche il primo significativo dossografo dell'eleate e per la sua ricezione si sono individuate fasi e tipologie diverse, cf. in proposito il fondamentale J. Palmer, Plato's Reception of Parmenides, O.U.P. Oxford 1999. Ippia e Gorgia sono giunti in forma frammentaria e comunque non hanno prodotto un'influenza rimarchevole. Nemmeno si può interpretare il termine come aggettivo o apposizione nel senso di una autoqualificazione di "ricco di canti", al pari dell'aedo Terpiade ( $O d .22,376)$, poiché non trova nessun riscontro
} 
si potrebbe trattare di nome proprio e non di aggettivo. Sulla linea della prima ipotesi si potrebbe arguire che Parmenide volesse indicare con certezza una strada mediante la menzione del nome che sarebbe verosimilmente apparsa nella sua intitolazione, cioè di colui al quale essa avrebbe potuto essere dedicata, il che sarebbe suffragato dal ritrovamento ad Atene di una via dedicata a un personaggio con il nome in questione. ${ }^{28}$ La poderosa dose allegorica e l'innegabile atmosfera d'interiorità che il contesto promana, sembra scoraggiare una tale interpretazione troppo 'materiale' e di dettaglio toponomastico poco pregnante, anche perché la via in questione per la sua unicità assoluta e imparagonabile non può ricevere una simile operazione toponomastica come una qualsiasi strada abitualmente frequentata. Infatti, non è questione di una qualche sua dimensione meramente 'terrena'; nemmeno può essere intesa come via dell'oltretomba per il semplice fatto che l'uomo che sa, cui essa in qualche modo è connessa, non può identificarsi con un defunto, anche perché essa è chiaramente rimasta umanamente imbattuta fino alla vicenda dell'evento parmenideo $(\mathrm{B} 1,18)$.

Dall'analisi si può dedurre la scarsa pertinenza contestuale di indicare un nome proprio con il lemma in questione, che invece trova una più adeguata luce se posto in una referenza culturale più ampia. Tuttavia, come aggettivo attribuito a una strada, da quanto è attualmente reperibile, si troverebbe solo nel verso parmenideo - non contrasterebbe con l'attitudine a volte mostrata di introdurre apax o neologismi, che però non riguarda specificamente il suddetto caso, visto che il lemma sicuramente raro e aulico è già presente altrove. Analogamente l'aggettivo in questione è reperibile due

anche se non si può però non ritenere che Parmenide non abbia acquisito una certa formazione anche approfondita di vari contenuti e veicoli culturali. Non è sconosciuto del tutto come aggettivo attribuito a бoфó De ebriet. I 371. Sarebbe ipotizzabile allora che Parmenide nell'attribuirsi l'aggettivo

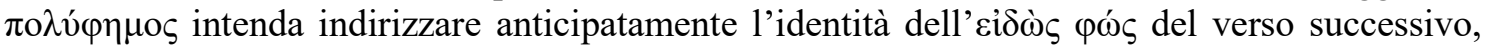
un'associazione alquanto peregrina poiché Parmenide ancora non è un tale sapiente e inoltre a differenza del contesto filoniano i due aggettivi sembrano non proprio reciprocamente allineabili. Occorre anche considerare se si tratti di un caso del fenomeno retorico d'ipallage, ossia con $\pi$ o $v_{\varphi} \eta \mu$ os si intende qualificare i1/la $\delta \alpha i ́ \mu \omega v$ e non la sua via. Va esclusa una tale proposta poiché non c'è traccia che l'aggettivo sia connesso con qualche divinità.

${ }^{28}$ Cf. A. E. Raubitscher, Dedications from the Athenian Acropolis, 1949, 259. Si potrebbe con molta immaginazione pensare che Parmenide fosse andato ad Atene per formarsi culturalmente e lì abbia avuto l'intuizione della rivelazione-dimostrazione dell'essere, notizia forse solo tramandata oralmente fino a Platone e poi manipolata da lui per includere la menzione di Zenone nel famoso incontro con Socrate nel Parmenide. Tale ipotesi sembra davvero singolare e soprattutto senza nessun appiglio dossografico. Se si riuscisse a dimostrare che Parmenide intendesse alludere proprio alla strada urbanistica di Atene, città reputata come centro ecumenico del sapere umano, si avrebbe un'autotestimonianza a conferma della narrazione platonica del suo presumibile viaggio nella suddetta città. 
volte però in connessione con ảyopá dal senso alquanto ovvio, cioè "dalle (o con) molte voci". ${ }^{29}$ La strada parmenidea sembra deserta, se le cavalle molto assennate e forse le giovani non vi si trovassero. Non pare che il senso 'dalle molte voci' come tradotto da $\pi \circ \lambda u ́ \varphi \eta \mu \circ \varsigma$ si possa estendere ai versi delle cavalle, benché in questo caso si tratti di figure equine molto particolari e 'intelligentissime'; allora si potrebbe riferire alle giovani immortali che riempiono delle loro voci la suddetta via. Del resto, è da notare che non si accenna a discorsi o a canti durante il percorso che conduce alla via polifemica, mentre si descrivono con una certa dovizia i rumori degli assi e delle ruote del carro, che avrebbero anche materialmente impedito l'esecuzione di qualche discorso o canto. D'altronde, l'aggettivo ha l'aspetto di qualificare la strada come una connotazione specifica e strutturale, non dipendente da qualche effetto riferibile all'evento singolare del viaggio parmenideo. Inoltre la via è polifemica poiché sarà esplicitata dal discorso della dea e quindi risulta di natura universale in qualche modo attinente a una certa istituzione divina, non perché la divinità la escogita ma perché la custodisce debitamente e altrettanto debitamente ne dà conto.

Non rimane che intendere la via 'polifemica' come magniloquente, dal solenne irrevocabile parlare, nel senso di contenere o manifestare 'molta (decisiva) voce (parola, sentenza)', di cui gli uomini sono rimasti del tutto all'oscuro perché sono mancati di incontrarla ${ }^{30}$, contravvenendo al proprio $\theta v \mu$ ó $\varsigma$ e 'follemente' abbandonandosi alla

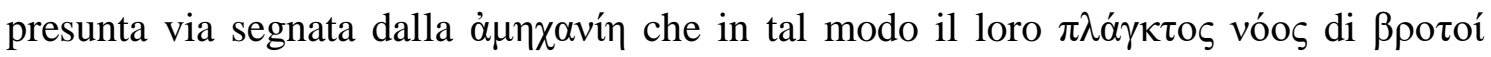

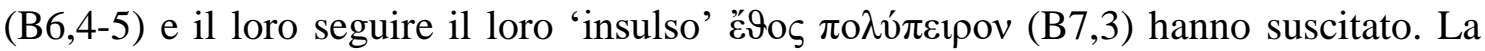
designazione della via come $\pi$ o $\hat{v} \varphi \eta \mu$ os significa la sua unicità aletica e la sua assoluta inconcussa funzione di far approdare alla verità, che non può non riguardare anche lo stato attuale umanamente antitetico e da dimostrare infondato, dipendente

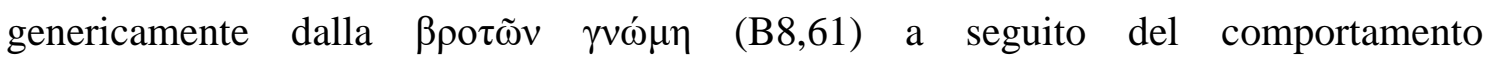
epistemicamente scorretto finora da tutti tenuto. L'aggettivo quindi denota la condizione di unicità della via per la sua esclusiva polifemicità (espressività assoluta di vero razionale in tutti i sensi possibili), segnale che la via è l'unica che può far conseguire la

\footnotetext{
${ }^{29}$ Od. II 150 ed Herod. V 79.

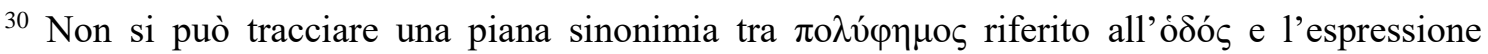

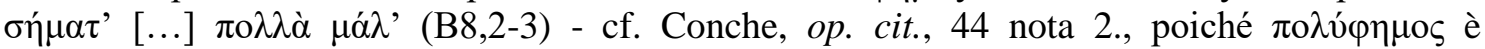
proletticamente funzionale e quindi a definire formalmente la via come l'unica abilitata a manifestare la verità, che attualmente solo lo speciale iniziato conosce per essere il solo tra gli esseri umani ad averla percorsa e quindi a riconoscervi la connotazione polifemica assoluta.
} 
verità $^{31}$ in modo da implicare anche il rifiuto o l'abbandono consapevole della escogitata via antitetica ingannevole. La via divinamente definita e presidiata è quella (logica e reale) della verità infallibile ma anche definitivamente 'sanzionatoria' rispetto al tentativo 'infelice' umana di ignorarla e in qualche modo indirettamente di 'sovvertila' con alternative impossibili. L'unico che l'ha potuta raggiungere, è anche l'unico che ha potuto conoscere la verità che presenta nel poema, e ha potuto quindi dare voce autentica alla sua polifemicità, dandone vero conto lungo l'esposizione. La via in esame è l'unica polifemica, cioè contenente in sé l'unicità semiotico-logica del vero sapere, la sola aleticamente concludente e tesa a distogliere razionalmente da quella illusoria di cui si deve dare conto in termini relativamente veri. L'aggettivo quindi sta a precisare l'autenticità indubitabile della via, l'unica che presenta il contenuto comunicabile con la noeticità umana. In tal modo si evidenzia l'incessante continuità tra il biografico immanente dell'espositore umano e l'inalterabilità universale e trascendente dell'unica dimensione aletica. Parmenide per entrare totalmente in questa dimensione (o per evitare il rischio anche per lui di essere soverchiato dall'universale stato di erranza da essa) è destinato a essere munito anche di mediazioni 'soprannaturali' o non ordinarie (cavalle, carro e giovani di origine divina, che aprono la

${ }^{31}$ Indubbiamente la distinzione enucleata da Prozdnev tra un senso passivo dell'aggettivo $\pi \mathrm{o}\langle\dot{\varphi} \varphi \mu$ os rintracciabile in Pindaro e uno attivo da assumere in Parmenide ("De Parmenidis fragmento B 1,3 DK", Hyperboreus 2001, 7 (1-2), 95 nota 26) risulta alquanto speciosa, soprattutto se si considera anche la nota critica contro Tarán che sostiene " $\pi$ o means 'famous' in ancient Greek literature" (op. cit., 10). Una traduzione che contempli solo l'aggettivo 'famoso', come in $D K$ e in Guthrie (op. cit. nota 8), non è sufficiente. Cf. anche Hermann Fränkel che attribuisce al lemma il senso esclusivamente attivo (Wege und Formen frühgriechischen Denkens. Literarische und philosophiegeschichtliche Studien, Zweite, erweiterte Auflage, Munich 1960, 159 nota 4). Si dovrebbe trattare di una sorta di 'enattivismo' come congetturato dal cognitivismo contemporaneo, ossia la strada corrisponde al percorso

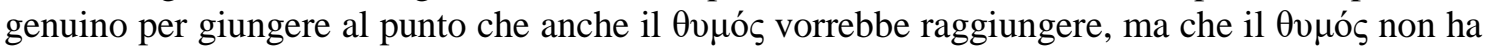
mai raggiunto ad eccezione di quello parmenideo. Non si tratta del contenuto aletico della via stessa o di quello doxastico derivante dalle varie opinioni inattendibili dei mortali, tra le quali potrebbero inserirsi anche il "much discourse" di natura pitagorica secondo Coxon (op. cit., 251) o anche secondo W. Jaeger (Paideia, Berlin ${ }^{2} 1936$, 240). Questi contenuti non paiono pertinenti con l'aggettivo in esame, che allude a quanto è rimasto contenutisticamente appunto ignoto, che presso i mortali non risuona affatto. Di tali temi non si accenna in questo esordio proemiale. Si tratta di dichiarare l'affidabilità assoluta della via a ogni livello e forma. Non si riesce a comprendere cosa dovrebbe entrarci il seguente ragionamento per dirimere la questione

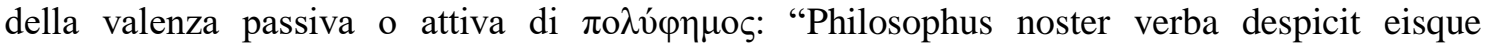

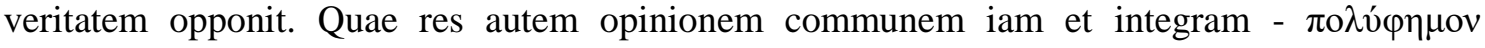
activum esse - abrogare haud potest. Nam etsi esset passivum (via. "de qua multum loquitur"), fama, quae a philosopho damnatur, a poeta nihilominus approbaretur." (cf. Prozdnev, art. cit., 95 nota 27$)$. 
strada cosmologicamente tratteggiata) ${ }^{32}$, che rappresentano un'iniziativa divina specifica e quindi infallibile. Le cavalle non sono solo mere trasportatrici (ă $\gamma \varepsilon v$ ) ordinarie per condurre all'esclusiva via del sapere per i mortali in genere, benché mai approcciata, anzi lasciata in una sorta di destino di impercorribilità e di intangibilità, ma sono soprattutto trasportatrici molto sapienti "fin dove l'animo raggiungerebbe", cioè sanno dove conducono per rendere raggiungibile il vero contenuto del sapere e il suo indefettibile e unico percorso per raggiungerlo; in tal modo mettono in grado di decifrare anche il senso vero della polifemicità della via, ciò per cui essa è in se stessa 'polifemica'.

\section{Il tormentato v. 3 del proemio}

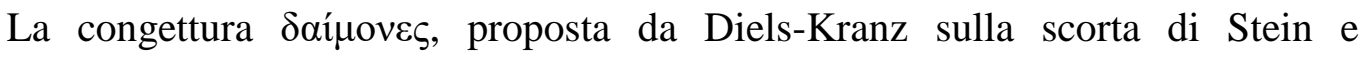
Wilamowitz $^{33}$, non è proprio verosimile e soprattutto non è rintracciabile in base a

32 È davvero singolare assegnare un ruolo inattivo alle giovani (Di Toro, L'enigma di Parmenide, cit. 113) che invece sono le sole mediatrici effettive e efficaci perché Parmenide sia introdotto nella sorte immortale di chi sa la verità infallibile e sa dove e come attingerla; è ancora più singolare vedere l'autore dietro la 'maschera' della innominata dea e addirittura che egli fosse già il sapiente perfetto di tutto ciò che la dea dovrebbe svelare mistericamente e non noeticamente.

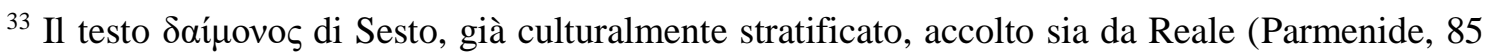
nota 3 con una qualche spiegazione del divino a pg 165ss) sia da Cerri (Parmenide di Elea, $167 \mathrm{ss}$ ), è l'unica lettura che ha base nei codici. Con le ipostatizzazioni teologiche o

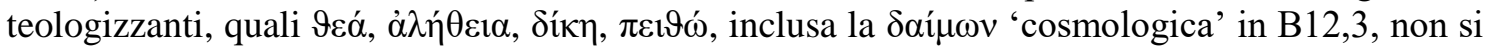
tratta tanto di impiegare un veicolo comunicativo diffuso, quale quello della mitologia, ma di dimostrarne la valenza aleteica che comunque persiste in qualche modo nella 'spiegazione' autentica o attinente alla verità che tocca inevitabilmente anche il cosmologico (doxastico). Il ravvisato contesto teologico di tipo enoteistico-monoteistico e di matrice orfico-pitagorica per cui il $\delta \alpha i ́ \mu \omega v$ addirittura sarebbe identificato con Zeus, come divinità suprema, secondo un parallelo presente in uno degli Inni Orfici (n. 73), dovrebbe spingere a far notare una discrasia radicale tra la nozione teologica parmenidea e quella orfico-pitagorica senza propendere per un concordismo alquanto forzato e miope. Nell'eleate non si può non supporre una qualche sostanziale sinonimia tra la $\vartheta \varepsilon \alpha ́$ in B1,22 (femminile non maschile come invece ci si aspetterebbe se si trattasse di concordismo orfico) e la già citata $\delta \alpha i ́ \mu \omega v$ in $\mathrm{B} 1,3$ (da interpretarsi al femminile di conseguenza), in modo da indurre a una sinonimia dei due termini in genere. Tuttavia sembra improbabile che a un certo punto la dea che svela tutt'intera la verità parli in terza persona a riguardo della struttura doxastico-meontologica secondo la dicotomia di essere e non essere. Ciò permetterebbe di coordinare la proposizione relativa immediatamente successiva non con "via", come quasi unanimemente si accetta, ma con il lemma immediatamente precedente, qualificando la divinità che porta l'uomo sapiente secondo la cultura mitico-teologica e non vera, benché universalmente celebrata e ritenuta autentica presso tutti i mortali, il che suffragherebbe ulteriormente che la via in cui Parmenide veniva trasportato dalle cavalle, si differenzi da quella che poi è percorsa fin dove l'animo arriverebbe e le giovani indicavano. Un simile prospetto sarebbe troppo criptico e soprattutto costituirebbe un certo 
qualche appiglio testuale - tanto meno la fantasiosa connessione con le non ancora menzionate кoṽ $\rho \mathrm{r}^{34}$, senza spiegare perché in tal caso non riferirsi piuttosto alle già menzionate ì $\pi \pi \mathrm{o}$, dato che non è inusuale l'attribuzione della natura divina a esseri animali speciali, come le suddette $i \pi \pi \mathrm{ot}^{35}$, e considerando che l'origine divina delle giovani è presto esplicitata in B1,9.

riconoscimento aleteico alla cultura mitica, che non si riscontra testualmente, benché essa sia da assumere come un certo sapere non eliminabile completamente nell'esposizione della verità

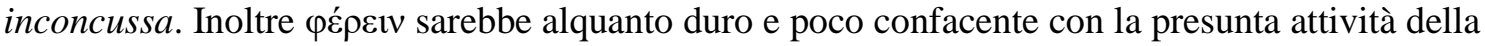
divinità ispiratrice di un contenuto religioso che nel poema si vuole piuttosto accantonare a favore della descrizione filosofica (necessaria) della verità autenticamente divina di tipo ontologico. Si deve quindi arguire che il percorso in questione è radicalmente irriducibile rispetto ai precedenti percorsi simili che rimangono al di qua dal punto che l'animo può raggiungere e in cui abita la verità nella sua assolutezza. Nulla vieta che con $\delta \alpha i ́ \mu \omega v$ in B1,3 si preluda all'unica divinità (volutamente anonima?) femminile che possa collegarsi con la stessa unica via 'polifemica' che, nonostante tutto, è l'unica a garantire proprio che tale divinità poi effettivamente insegni (non tanto riveli) la verità dell'essere - cf. anche le osservazioni convincenti e risolutive in tal senso in Prozdnev, op. cit., 95-96 e nota 30, concordando con Tarán (op. cit., 11) e Coxon (op. cit., 157). La scelta del genitivo (soprattutto nella declinazione possessiva) con l'associazione evidenziata offre molto senso nel contesto, poiché simboleggia l'ineluttabilità dell'intervento divino (non generico) ma peculiare inerente alla stessa via che altrimenti non potrebbe aprirsi per le cavalle (e le guidatrici Eliadi che non si possono identificare con le Muse diversamente da come si è sostenuto) al momento in cui inizia il loro traino. Pertanto le cavalle dovrebbero solo portare trainando il carro e non proprio 'condurreguidare': benché siano molto scaltrite, devono comunque essere condotte da una guida adatta. En passant, Parmenide si riferisce a un linguaggio e a una cultura iniziatica, non per adottarla nella nuova veste di discepolo della dea, ma per opporsi a essi o differenziarsi da essi, poiché quanto si conseguiva secondo la ritualità orfica non portava alla via vera, ma a quella falsa, cioè al sapere umano infondato. Parmenide non è nemmeno in alcun bivio, come si è insinuato, lasciandosi prendere troppo la mano dalle suggestioni dei paralleli orfici (Maria Michela Sassi, "Parmenide al bivio. Per un'interpretazione del proemio", Parola del Passato 43, 1988, 387357, 387), in sostanziale sintonia con Pugliese Caratterelli, citato nella nota precedente qui) ma è introdotto nell'unica via, quella non rapsodica e rapsodicamente raffigurata. Essa è quella non apparente in contrapposizione proprio a quella fittizia che nemmeno rimane "in tutte le città" o

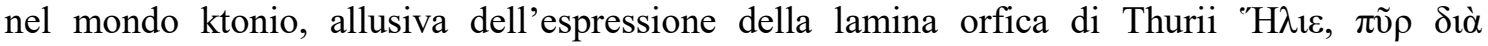
$\pi \alpha v \tau^{\prime} \not \alpha \sigma \tau \eta v 1 \sigma \varepsilon \alpha \mathrm{l}$ in fr. 57 Kern. Un simile parallelo con B1,3 non solo è dubbio in sé ma anche la relativa ricostruzione epigrafica è alquanto inventata. Non incoraggia, d'altro canto, quell'artificiosa proposta da Giovanni Cerri in Parmenide di Elea. Poema sulla natura (Milano 1999), 147; cf. anche la discussione filologica in M. Prozdnev, op. cit., 91-101). Nemmeno si può sostenere con la Sassi che la via alla e nella quale le Eliadi conducono sia quella dell'oltretomba, metafora del processo e percorso iniziatici, cioè sia rappresentabile in termini dell'immaginario orfico, poiché proprio questa è al limite la presunta via in cui Parmenide stesso si sarebbe inoltrato o sarebbe stato introdotto nella modalità antropico-doxastica. Parmenide intende proprio escludere a priori una simile via come il comune percorso culturale gnoseologicamente infondato (anche se a suo tempo e nel modo dovuto dovrà essere affrontato).

${ }^{34}$ Vedi l'apparato critico D-K 228.

${ }^{35}$ La divinizzazione di animali specialmente di razza equina non è del tutto inedita se si rammentano Xanto e Balio, mitici cavalli immortali di Achille. L'assenza di qualsiasi accenno a una qualche loro facoltà linguistica delle cavalle parmenidee, a differenza dei cavalli omerici, e addirittura di preveggenza attribuita a Xanto, costituisce un elemento piuttosto di discontinuità con l'immagine omerica, con cui si raffigurano piuttosto 'esteriormente' animali da trazione non 
Se quindi non si può ravvisare una differenza significativa tra $\delta \alpha i ́ \mu \omega v$ e $\vartheta \varepsilon \alpha ́$, non c'è nessuna ragione accettabile per cui non si dovrebbe identificare la $\delta \alpha i ́ \mu \omega v$ in B1,3 con la Э\&á di B1,22 - poco importa la motivazione basata sull'osservazione che a tale figura teologica è riservata la mera anonimia, poiché è sic et simpliciter la 'dea della via polifemica' o a essa preposta. L'obiezione che il lettore, cui tale $\delta \alpha i ́ \mu \omega v$ non sarebbe ancora presentata come la successiva $\theta \varepsilon \alpha$, non potrebbe immaginare che in tal modo ci si stia riferendo a lei, non regge molto per la sua connotazione alquanto anacronistica. Il contesto infatti lo rende immediatamente evidente. Se la via è quella polifemica, essa non può non essere quella che appartiene alla divinità specifica, preposta a dimostrare (più che a rivelare) la verità tutta intera all'iniziato (e a ogni possibile uomo che in tal modo si può definire conoscitore di tale verità). Si potrebbe intendere, certamente, la divinità in modo generico, ma questo sarebbe superfluo e poco indicativo, oltre al fatto che si dovrebbe giudicare un maschile stridente con l'inobiettabile 'femminilismo' parmenideo.

L’interpretazione della $\delta \alpha i ́ \mu \omega v$ nei termini appena esposti crea un altro problema esegetico. Il susseguente pronome relativo ì potrebbe a questo punto essere riferito proprio al precedente $\delta \alpha i ́ \mu o v o \varsigma$ giustificando ancora più inequivocabilmente l'assunzione del tipo di genere di tale lemma, ma anche i tratti peculiari e inconfondibili della dea appunto, l'unica che porta ( $\varphi \varepsilon ́ \rho \varepsilon \imath)$ un qualche mortale al sapere, ossia con la sua azione didattica infallibile che si sta per realizzare e già realizzato rispetto a chi narra. Tale associazione sintattico-semantica non pare peregrina anzi abbastanza attestata, però nel contesto parmenideo risulta azzardata, anche perché il passo in esame si focalizza sull'autenticità e direzionalità efficace della via e non sulla $\delta \alpha i ́ \mu \omega v$, che è introdotta per esplicitare la qualità assoluta della via stessa. Tuttavia, una simile ipotesi potrebbe ricevere proprio un'obiezione nel considerare che ä $\tau \eta$, il lemma tanto oscuro ma l'unico testualmente accertato con il codice più antico (XIII sec.), e rispetto a tale lezione fino al XV sec. non si registrano da rilievi ecdotici variazioni codicologiche ${ }^{36}$ né

impegnati a portare verso la via della conoscenza umana, fin dove appunto l'animo può giungere. È quanto si intende enfatizzare con il probabile neologistico $\pi 0 \lambda \hat{\varphi} \varphi \rho \alpha \sigma \tau o l$ ad esse attribuito subito dopo nel verso successivo.

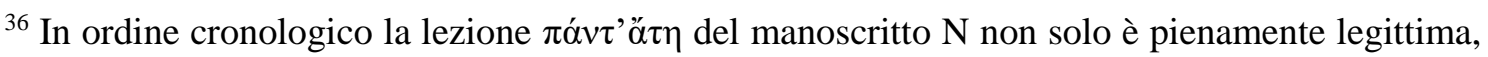
ma anche la più contestualmente sensata. La sua trasmissione variata (corrotta) inizia con $\pi \alpha ́ v \tau \dot{\alpha} \tau \eta$ del $\operatorname{cod}$. L, che pare obliare lo spirito e l'accento sulla trascritta $\alpha$. Si passa alla lezione

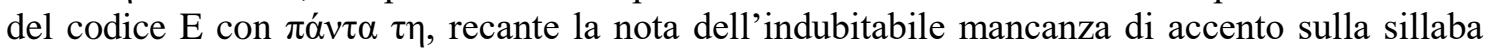
$\tau \eta$. Infine i codici $\varsigma$ con l'implausibile accentazione su tale sillaba presenta la variazione

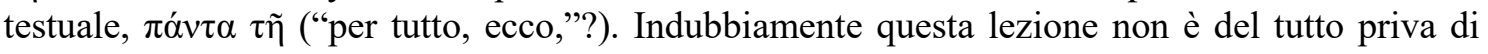


attestazioni filologiche di ipotesi di corruttela ${ }^{37}$, situazione perlopiù ancora immutata (a parte qualche eccezionale voce discordante) a partire dalla seconda metà del sec. XIX

senso però è un po' dura e soprattutto 'rimediata'. La sua traduzione nel contesto potrebbe essere: "la quale ( $s c$. via) in tutto (interamente, in assoluto), ecco, porta l'uomo che sa". In qualche modo si vuole enfatizzare con "ecco" che quanto si sta per narrare e discutere non è altro che l'essere portato da questa via, e finalmente adesso è disponibile.

${ }^{37}$ L'ipotesi di corruttela, che è ereditata in modo del tutto acritico a partire del XVI, si trova nella prima edizione parziale dei frammenti parmenidei ma contenente l'intero testo proemiale sestiano, ovvero "Parmenidis Carmina", in Poesis Philosophica, [...], a cura di Estienne Henry (Henricus Stephanus), Genève 1573, 41-46, riporta per la prima volta sulla base dei codici posteriori un tentativo di ricostruzione filologica con una simile resa: $\pi \alpha ́ v \tau$ ' $\alpha \tau \eta \varphi \varepsilon \rho \varepsilon 1$ (cf. $i b .41$; senza accenti). L'assenza di inserimento degli accenti sull'ultimo termine avvertito come un unico lessema nasconde una incertezza la cui causa si è definitivamente oscurata e va ravvisata proprio nel deterioramento della trascrizione degli accenti giusti. La seconda edizione dei Chouët (op. cit., 157), riprende la versione ulteriormente corretta dei codici peggiori ( $\varsigma$ ) con

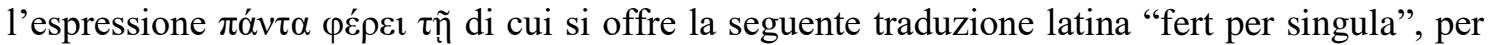
cui l'avverbio $\tau \tilde{\eta}$ in qualche modo è avvertito come una forma di rafforzativo e dimostrativo del pronome relativo femminile riferito a ódó $\varsigma$ - il suddetto sintagma viene introdotto con "atque via quae" in Fabricius, op. cit., 393, in cui si riporta però la versione greca di Stefano (Henry)

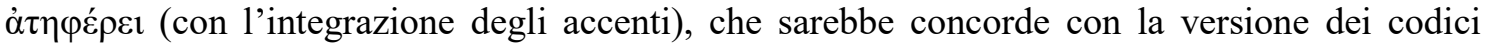
Saviliano e Vratislaviense, impiegati da Fabricius secondo ciò che si dà a sapere con la nota in calce $E$ (ibidem) specificando che Stefano giudica corrotto il luogo. Si suggerisce a supporto della versione riportata: "Possit autem videri $\alpha \tau \eta \varphi \varepsilon ́ \rho \omega$, si sana sit lectio, verbum formatum ut $\grave{\alpha} \tau \eta \mu \varepsilon \lambda \varepsilon \dot{\varepsilon} \omega . "$, ipotesi improponibile. L'incomprensione del testo originario in parte contrastato o trascurato dall'edizione chouetiana che si avvale di codici recanti manomissioni correttive, è stata suscitata o raccolta e rilanciata da Stefano, benché. Si sono succedute 'infinite' congetture, alcune anche acute ma sostanzialmente poco convincenti nel loro insieme. Georg Gustav Fülleborn propone àï̇̃̃, in Parmenidou Tou Eleatou Leipsana. Fragmente Des Parmenides. Gesammelt, Übersetzt und Erläutert von Georg Gustav Fülleborn. Zûllichau 1795, 36, certo della corruttela del luogo secondo il citato Justus Joseph Scaliger, come premesso (anche se costui annota soltanto: "pag. 41, vers. $\Delta \alpha i ́$ ovo $]$ ] Locus corruptus", in Poesis Philosophica, cit., 217). La congettura non è ammissibile per diverse ragioni e non ultima per quella concernente l'accento. Si vieta assolutamente di poter assumere la lezione di ö́tᄁ: "Ne cui forte in mentem

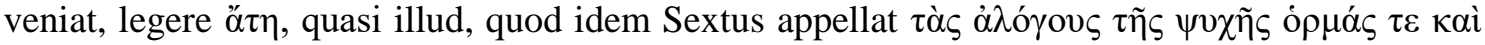

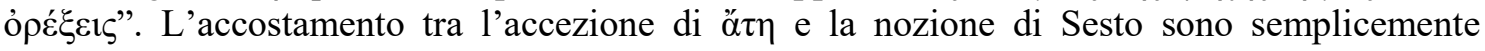
incompatibili e in ciò si può essere d'accordo con Fülleborn. L'anno successivo Chr. G. Heyne, recensendo la sua opera appena citata, ha congetturato un'ulteriore lettura öv $\tau \eta v$ in "[Rec.]: Fragmente des Parmenides. Ed. G. G. Fülleborn”, Göttingische Anzeigen von gelehrten Sachen

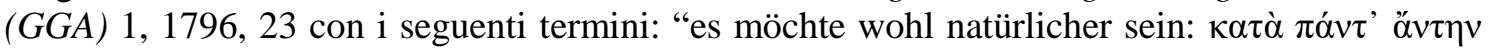

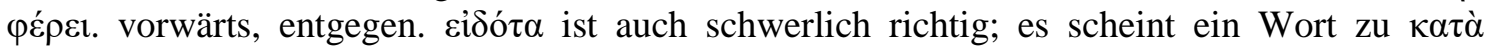
$\pi \alpha ́ v \tau \alpha$, wie $\check{\varepsilon} \sigma \beta \alpha \tau \alpha$, ̋̌ $\mu \beta \alpha \tau \alpha$, erfordert zu werden". Benché l'accento sia più plausibile e si rispetti la consonante $\tau$ (filologicamente attestata) invece dell'infondata $\delta$, non si riesce a capire la necessità di integrare con la geminazione di $v$ disposta in sintonia con un termine che non ha alcuna base testuale e che di recente è stata ripresentata con il corredo di tentativi probativi in base a presunti parallelismi omerici in The Fragments of Parmenides. A critical text with introduction, translation, the ancient testimonia and a commentary by A. H. Coxon, Assen ${ }^{1} 1986,45,158$ - sulla ampia e un po' oziosa discussione in proposito si rinvia a Prozdnev, art. cit., 93 e note 17-19. Per senso di completezza, si rammenta solo la proposta di $\sigma \alpha \varphi \tilde{\eta}$ ("ad manifesta quaequae") in Commentationum Eleaticorum pars prima, sectio secunda. De Parmenide. Exposita a Chr. Aug. Brandis, Altonae 1813, 93: con il commento "sensu emphatico". La variante $\alpha \delta \alpha \tilde{\eta}$ ("arcana" tr. lat.) in Parmenidis Eleatae carminis reliquiae. De vita eius et studiis disseruit, fragmenta explicavit, philosophiam illustravit S. Karsten, 
che ha prodotto un ulteriore fiorire delle più disparate congetture o di riprese di quelle

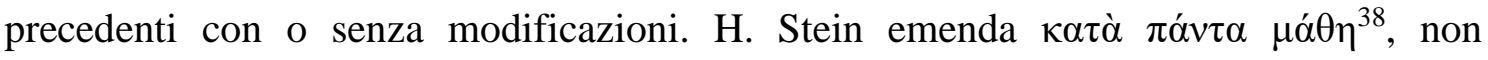
sostenibile per diverse ragioni, tra le quali quella più determinante consiste nell'assenza assoluta di appigli testuali ad eccezioni degli accenti, oltre al fatto che essa risulta alquanto pleonastica o tautologica (a cosa dovrebbe portare la via 'numinosa' se non almeno alla verità tutt'intera e 'rotonda'? E se porta l'uomo che sa, come potrebbe essere un tale sapere deficitario di qualcosa o incompleto?). Bergk, dopo avere emendato, come già esaminato, con $\kappa \alpha \tau \alpha ̀ ~ \pi \tilde{\alpha} v \pi \alpha ́ v \tau \eta$ il primo sulla scia della proposta di

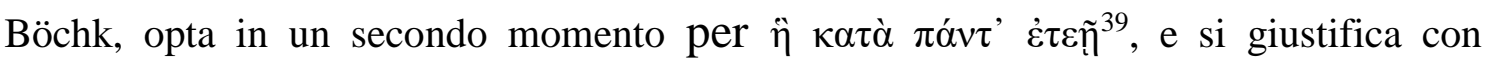

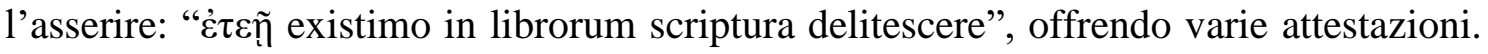

Amstelodami 1835, 55, recepita da F. Riaux, Essai sur Parménide d'Elee, suivi du Texte et de la Traduction des Fragments, Paris 1840), forse ricavata da B8,59 in contrapposizione al criticato ăï̇̃̃ di Fülleborn, è inammissibile per diverse ragioni e dal punto di vista testologico per

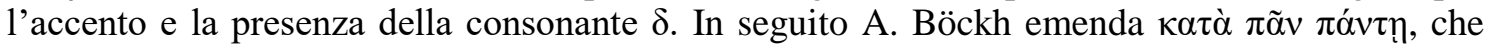
pare un errore dittografico per allitterazione, in Parmenidis libri de natura exordium emendatur, Berlin 1836 (ristampato in Gesammelte kleine Schriften, Lipsiae, vol. IV), 416, appena variato

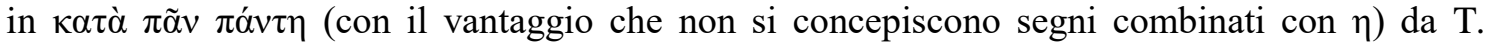
BERGK, "Commentatio de Empedoclis prooemio" [1839], Kleine philologische Schriften II,

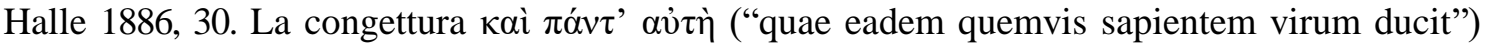
riferita a G. Hermann, poiché non è stato possibile reperire il testo originale, a partire dalla edizione di Sesto Empirico a opera di I. Bekker (cf. Sextus Empiricus ex recensione Immanuelis Bekkeri, Berolini Typis et Impressis Gr. Reimeri A. 1842, 26, app. cr. 11), che annota "qui кaì

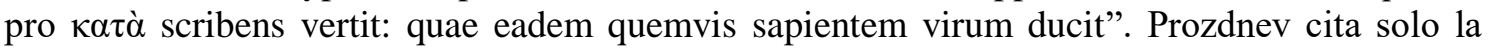
dissertazione di Hermann sul vocabolo av̉ ós (cf. art. cit., 100 e nota 66) aggiungendo: "Antitheto enim sententia Parmenidis struitur: per omnia eadem sapientem virum fert via.", una traduzione che non concorda totalmente con quella offerta da Bekker già trascritta. In tale testo si riporta la seguente espressione di Hermann: "Alius apud Homerum usus nominis av̉ ò̀ ille est, quum significandae caritatis caussa aliquis ipse aliquid fecisse dicitur: [...]" (Opuscula 1, Lipsiae 1827, 313). Dei quattro passi omerici menzionati il primo (Il. V 734) il terzo (Il. XV 198) e il quarto (Od. XIV 141) si trascrivono, a differenza del secondo (Il. VI 385) che non presenta il vocabolo in questione. Si conclude con tale asserzione: "Et huius quidem generis exempla extant plurima". Da tale panoramica si può inferire che Hermann non dovrebbe giustamente valutare il verso parmenideo come un esempio di tale uso del pronome testualmente congetturato ma non attestato. L'emendamento con $\dot{\alpha} \sigma ı \tilde{\eta}$ è riferito a Meineke per la prima volta nell'apparato critico di Bekker nella sua edizione sestiana appena citata. Per un

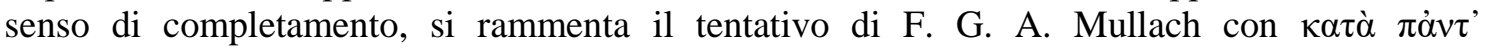

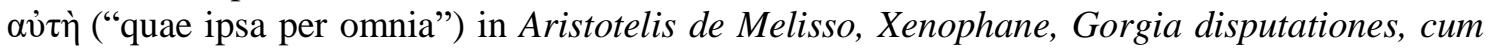
eleaticorum philosophorum fragmentis, Berlin 1845, ripetuto in "Fragmenta philosophorum Graecorum [vol. 1]", Poeseos philosophicae caeterorumque ante Socratem philosophorum quae supersunt / collegit, recensuit, vertit, annotationibus et prolegomenis illustravit, indicibu[s] instruxit Fr. Guil. Aug. Mullachius, Paris 1860, 114.

38 "Die Fragmente des Parmenides $\pi \varepsilon \rho \grave{~} \varphi v ́ \sigma \varepsilon \omega \varsigma$ ", Symbola philologorum Bonnensium in honorem F. Ritschl, Lipsiae 1863-1867, 772.

39 "Emendationem Parmenidearum Pars I" [1864], Kleine philologische Schriften II, Halle 1886, 68. La congettura fu accolta da H. Usener, "Variae lectiones specimen primum", Jahrbücher für classische Philologie 35 (1889), vol. 139, 369-397, 383. 
Si aggiunge che la scelta di una tale 'scappatoia' filologica vale come una sorta di

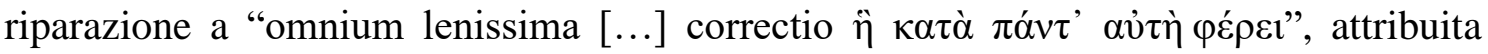
erroneamente a Hermann (ma in effetti formulata da Mullach), e si critica: "nimis otiosum est illud pronomen", critica che non può non essere sottoscritta. Questa lettura è inammissibile per diversi motivi e soprattutto per l'eccessiva variazione lessicale del sintagma senza alcuna ragione testuale, oltre alla superfluità della specificazione éđ̇ñ.

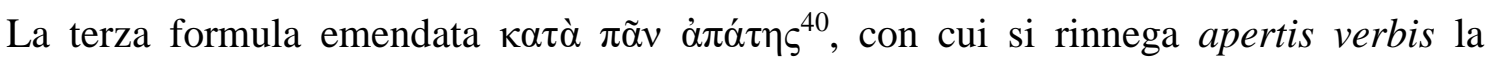
seconda, non mostra nessuna novità significativa. Non si intende focalizzare nel testo originario che la via sia il metodo indicato per conoscere e ovviare a errori o potenziali deficienze intellettive, poiché il punto da chiarire verte su una specificazione essenziale della descrizione della via. Infatti, si spiega quest'ultima opzione di ricostruzione filologica: “i. e. quae per omnes errores et sensuum fallacias virum ita ducit, ut mentis acies ne hebescat". Del resto l'uomo che sa perché vi è condotto non può temere una simile eventualità come chi in genere non vi è condotto e quindi non sa semplicemente.

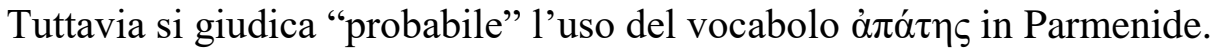

Il terreno ormai completamente minato e volto a interpretazioni quasi 'a ruota libera' ha prodotto un emblematico quanto singolare fenomeno costituito da continui ripensamenti come si riscontra nel già citato Diels, il quale pur lasciando la forma corrotta $\pi \alpha ́ v \tau \alpha \tau \eta$ nel testo della sua prima edizione ${ }^{41}$, si perita di giustificare

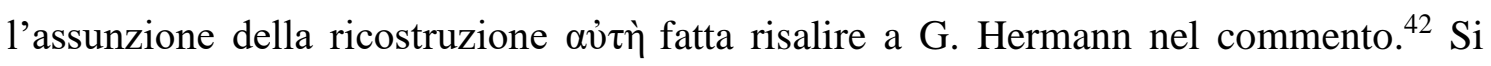
incontra subito dopo la ricostruzione di Patin, che con una perizia testologica alquanto

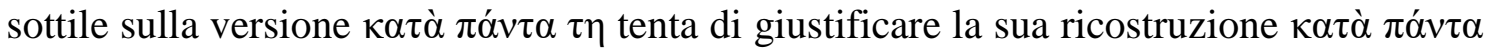

\footnotetext{
40 “Emendationem Parmenidearum Pars II, ibid., 77.

${ }^{41}$ H. Diels, Parmenides Lehrgedicht, Berlin 1897, 36 citando già nell'apparato critico la ricostruzione di Hermman che sarà parzialmente giustificata nel commento di seguito (cf. nota seguente).

${ }^{42} I b$., 48, in cui si scrive: "Zahllos sind die Emendations-versuche der verderbten Stelle [... si riportano le varie proposte più rilevanti fino allora note]. Man könnte noch einige Einfälle

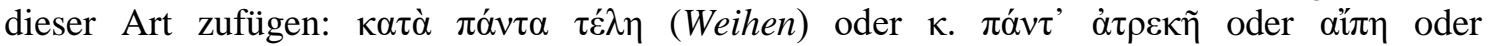

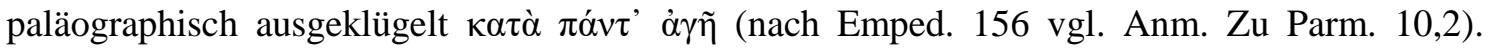
Wahrscheinlich erscheint von allen bisher Vorgeschlagenen nur G. Hermanns av่

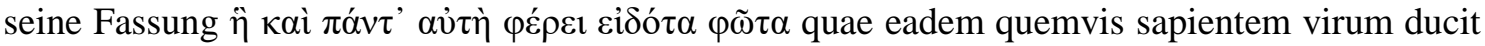

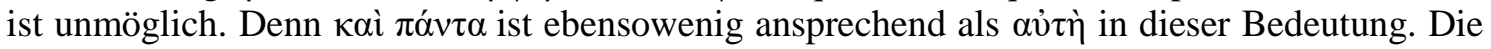

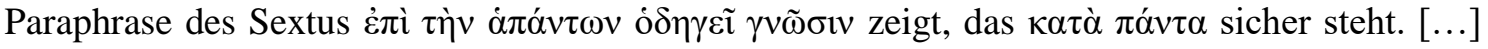
Ich erkläre sola: der göttliche Weg, der allein den wissenden Mann überallhin führt". Che il

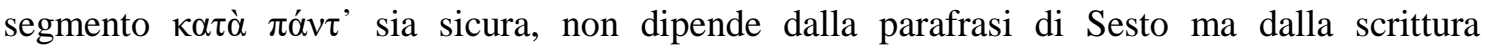
estremamente chiara (e incorrotta) del codice $\mathrm{N}$; inoltre sola (allein) è talmente irrilevante nel contesto che non varrebbe la pena congetturarlo.
} 


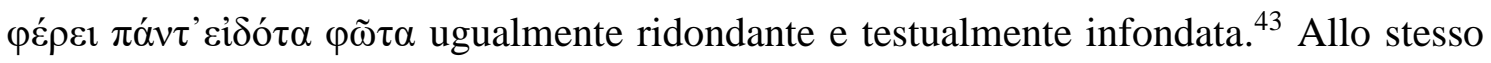
anno risale la congettura di Wilamowitz $\kappa \alpha \tau \alpha ̀$ $\pi \alpha ́ v \tau \alpha ~ \tau \alpha \tau \eta^{44}$, che è inammissibile a partire dall'accento, oltre al fatto che sottintende una valenza di apertura generica, come si suggerisce esplicitamente con i seguenti termini: "wir verlangen eine via per omnia patens". Il Diels nella sua seconda edizione continua a lasciare nel testo la forma corrotta $\pi \alpha ́ v \tau \alpha \tau \eta$ e nell'apparato si limita a citare tra parentesi le sue proposte già menzionate, a cui aggiunge $\pi \alpha v \tau \alpha v \gamma \eta ́ s$, insieme alla congettura di Wilamowitz, benché concluda con la preferenza di quanto già asserito circa la proposta rivisitata di Hermann:

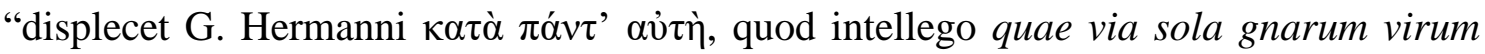
per omnia ducit ${ }^{\prime 45}$, che traspone direttamente nella trascrizione della prima edizione dei

43 A. Patin, "Parmenides im Kampfe gegen Heraklit", Jahrbücher für classische Philologie Suppl. 25, 1899, 648, in cui si sostiene: "so viele wollte das heillose $\tau \eta$ umgestalten - und sie

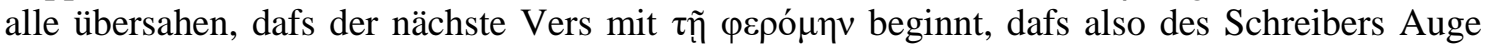

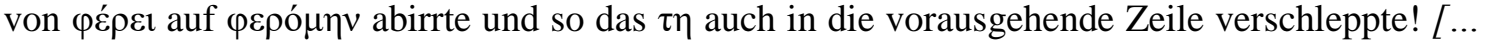
si riportano note critiche significative su alcune altre congetture che terminano con quelle

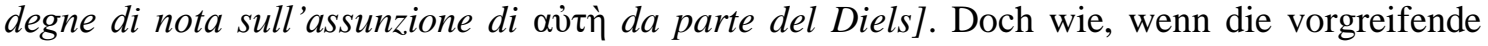
Dittographie $\tau \eta$ eine Lücke hinter $\varphi \varepsilon ́ \rho \varepsilon ı$ veranlafst hätte und diese durch $\pi \alpha ́ v \tau$ ' zu ergänzen wäre, so dafs nun der umgekehrte Fehler mitspielte, die Auslassung einer in einem Vers zum zweiten Male zu schreibender Silbe? Eben dieses zweite $\pi \alpha ́ v \tau$ ' konnte jenes Abirren des Auges veranlafst haben, das nun, als $\tau \eta$ verschleppt war, zur Heilung des Verses seinen Platz räumen mufste". Una duplice dittografia inversa è tanto fantasiosa quanto al limite dell'assurdo. Dal

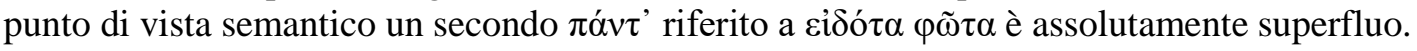

44 U. Wilamowitz, "Lesefrüchte", Hermes 34.2, 1899, 204. Poco chiara l'illustrazione

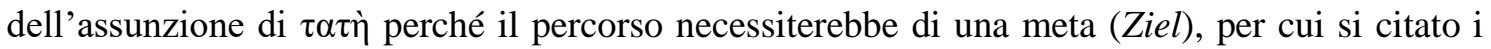
passi omerici di $I l$. XXIII 375. 758, il cui parallelismo con il passo corrotto (?) parmenideo è del

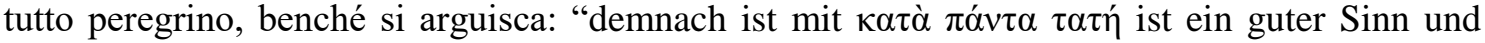
Vers und eine geringe Aenderung erzielt". Che il senso e il verso siano buoni e soprattutto l'alterazione sia minima, non è affatto garanzia che si sia risanata nel modo giusto la corruttela $o$ il testo che si giudica tale. Cf. anche le obiezioni e critiche da diversi autori, dei quali si citano W. J. Verdenius, Parmenides. Some Comments on his Poem, Groningen 1942, 66 e poi G. S. Kirk-J. E. Raven, The Presocratic philosophers, Cambridge ${ }^{1}$ 1957, 267-268 (totalmente obliata in g. S. Kirk-J. E. Raven-M. Schofield, The Presocratic philosophers, Cambridge $\left.{ }^{2} 1957\right)$. Cf. le note critiche in Prozdnev, art. cit., 94 e nota 22, in cui si asserisce l'accoglimento della proposta in H. Ritter - L. Preller - E. Wellmann, Historia philosophiae Graecae, Gothae ${ }^{9} 1913$, § 113; R. Eisler, Weltmantel und Himmelszelt, München 1910, 338, benché si ammicchi a un senso non traslato come in Wilamowitz, ma a uno proprio con riferimenti al passo di Aristotele sulla membrana (HA 519 a 32) e a scoli omerici ( $B 43 ; \Xi 185)$, oltre a uno indiretto in Soph. Oed. Tyr. 733-734. Se ne arguisce: "nulla obstat, quominus apud Parmenidem $\tau \alpha \tau$, "simplex" scilicet et "perpetua", dici possit."; ma saggiamente si conclude: "Difficilius diiudicari potest, aptumne $\tau \alpha \tau$ ' sensum hic praebeat. Nec satis percipio, quare viam suam indivisam poeta designaverit.", anche se tale indivisibilità è da dare per ovvio o comunque non sono reperibili attestazioni in senso opposto nel poema.

${ }^{45} \mathrm{H}$. Diels, Poetarum philosophorum fragmenta. Auctore Udalrico de Wilamowitz-Moellendorf Collecta et Edita, vol. III, ed. H. Diels, Berlin 1901, 58 (cf. app. crit. $\pi \alpha ́ v \tau \alpha \tau \eta$ in cui cita per la

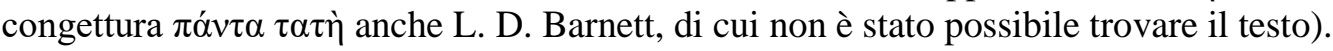


Die Fragmente e ne offre la traduzione tedesca. ${ }^{46}$ Cambia ancora idea e assume in tutto e per tutto la tesi Wilamowitz nella seconda edizione (la terza effettiva) ${ }^{47}$, per assumere

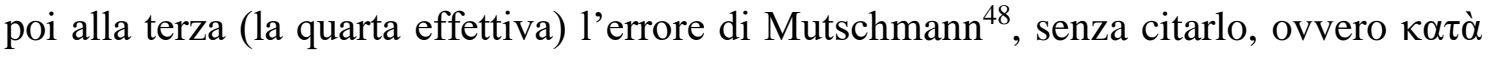
$\pi \alpha ́ v \tau$ ’ó $\sigma \tau \eta$ (“durch alle Städte”), che rimarrà definitiva nelle altre edizioni pubblicate con o dal solo Kranz. Nel relativo apparato critico dopo avere presentato le lezioni dei

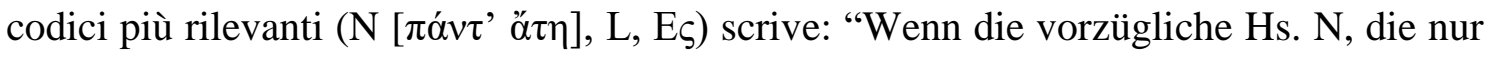
sehr selten interpoliert [... cita un altro passo a supporto], das Echte bewahrt hat [... cita un altro passo a supporto], so legt der Philosoph von vornherein Wert darauf [... si

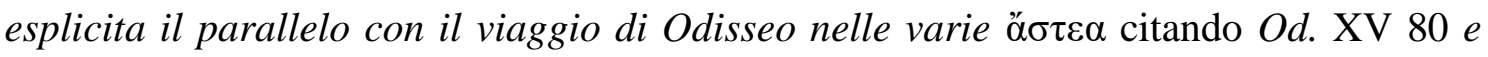

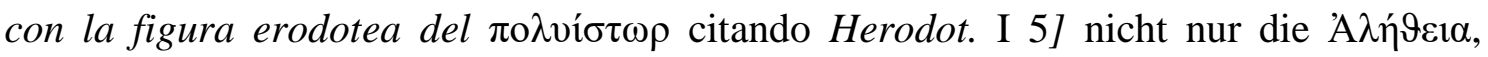

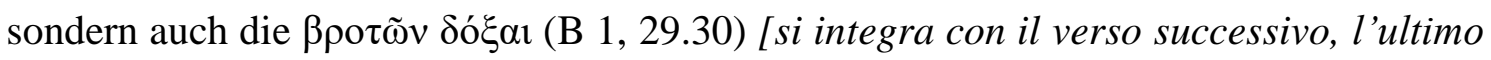
del proemio]". Si vuole enfatizzare il giudizio filologico sulla bontà indiscutibile del manoscritto $\mathrm{N}$ (nota che rimarrà sostanzialmente invariata nelle altre edizioni). Nonostante ciò ci si affida alla versione di un testo edito infondato e si tenta di motivare con parallelismi letterari poco istruttivi, a dir poco, anzi nella mente di Parmenide tale confronto avrebbe dovuto risuonare in netta antitesi rispetto al senso del suo testo (unico e su un'unica strada collegabile con la verità). Anche se la via parmenidea permette indirettamente a fortiori di conoscere la falsità delle ipotesi umane, inevitabilmente da svelare nella loro vera essenza di inconsistente doxasticità, per queste non sussiste nessuna vera via (gnoseologica), ma solo una ipotetica via che porta a erranze e quindi di per sé risulta realmente impercorribile, che la dea rende in qualche modo esponibile secondo la sua autentica fisionomia '(me)ontologica' in un sistema semiotico-verbale

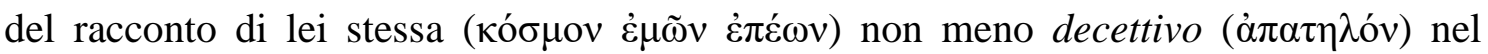
contenuto di come i mortali (confusamente e disordinatamente) le propongono. Inoltre cosa c'entra parlare di “città", anche se la nozione è letta nel senso metaforico per omnia o überallhin? Tuttavia, la stragrande maggioranza degli studiosi fino alla data fatidica del 1968, attinente alla dimostrazione ineccepibile di Coxon che questa proposta

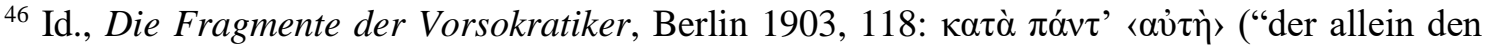
wissenden Mann überallhin führt").

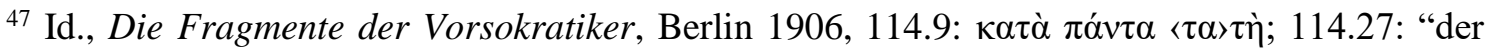
überallhin sich dehnende den wissenden Mann führt".

48 Id., Die Fragmente der Vorsokratiker, Berlin 1912, 148.16. La conclusione del relativo

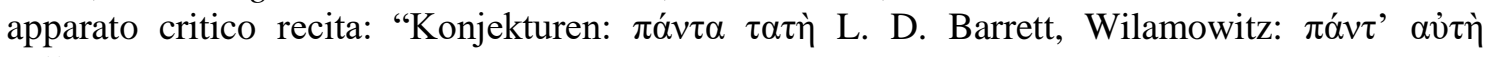
(allein) G. Hermann".
} 
sia di fatto una lectio falsa ${ }^{49}$, si sono attenuti a un simile dato filologicamente quasi inopinabile $^{50}$, ad eccezione di W. Jaeger che propone il non meno problematico $\dot{\sigma} \sigma \mathrm{v} \tilde{\eta}$ (unversehrt) ${ }^{51}$, in modo indipendente (a quanto si racconta $^{52}$ ) da Meineke. La nota di Coxon, oltre a riaprire la questione della corruttela con una serie di congerie di varie (più $\mathrm{o}$ meno modificate) edite $\mathrm{e}$ inedite congetture, soprattutto non è riuscita a scoraggiare insostenibili (e a volte patetici oltre che inutili) tentativi di supportare la

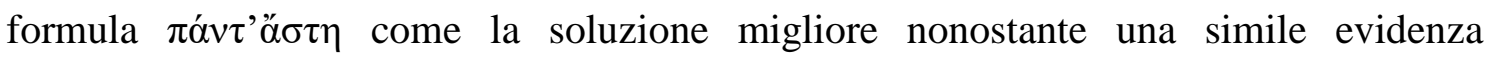
testologica di essere in presenza di un "falso". Per primo cronologicamente si incontra A. P. D. Mourelatos che non esita ad asserire di volersi attenere alla congettura tradizionale, definita "more than an emendation" e la più prossima al testo e da preferirsi all'altra unica sensata, ovvero ḋoı̃̃, dei citati Meineke-Jaeger ${ }^{53}$, e seguito dalla prima edizione di E. Heitsch. ${ }^{54}$

${ }^{49}$ A. H. Coxon, "The text of Parmenides fr. 1,3", Classical Quarterly, 18, 1968, 69, per una probabile riproduzione delle lettere sottolineate in $\pi 0 \lambda \hat{\varphi} \varphi \underline{\alpha \sigma \tau o l}$ del verso successivo, per cui, a

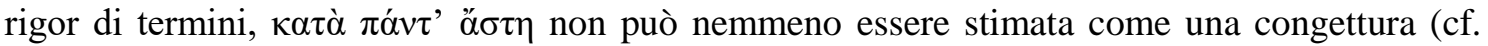
Prozdnev, "De Parmenidis ...", 91), ma semplicemente un errore di trascrizione dovuto a H. Mutschmann (op. cit., 26).

${ }^{50}$ M. Bowra, “The Proem of Parmenides”, Classical Philology 32, 1937, 97-112, 109, seguito da P. Albertelli, Gli Eleati. Testimonianze e frammenti, Bari 1939, 122-123 nota (7), in cui si

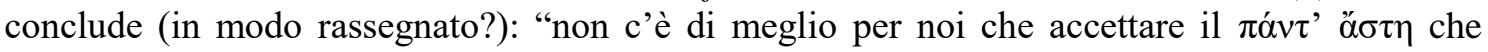
almeno è in un ms. e per il quale Bowra [si rinvia al luogo appena riportato] cita l'orfico "H $\mathrm{\lambda} 1 \varepsilon$

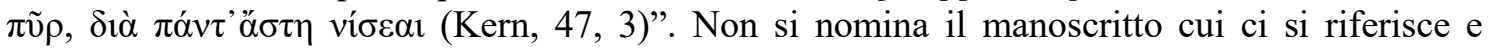
inoltre, anche se fosse, dovrebbe applicarsi il criterio textus unius textus nullius. Non si sospetta nemmeno che l'orfico verso della lamina possa essere una mera congettura, oltre a riservare altri punti critici. Cf. anche M. Pohlenz, Herodot. Der erste Geschichtschreiber des Abendlandes, Leipzig 1937; C. De Vogel, Greek philosophy; a collection of texts with notes and explanations, vol. I, Leiden 1950; J. Zafiropoulo, L'école Eléate. Parménide, Zénon, Melissos, Paris 1950; H. Fränkel, Dichtung und Philosophie des frühen Griechentums. Eine Geschichte der griechischen Epik, Lyrik und Prosa bis zur Mitte des fünften Jahrhunderts, New York 1951; G. S. Kirk-J. E. Raven, op. cit., 267s; M. Untersteiner, Parmenide. Testimonianze e frammenti, Firenze 1958, LII nota 4 e CLXXI nota 52; L. Tarán, op. cit., 10ss.; W. K. C. Guthrie, A History of Greek Philosophy, II, Cambridge 1965.

${ }^{51}$ Paideia; die Formung des griechischen Menschen, 3 vols., Berlin 1933-1947, 175 n. 35.

${ }^{52}$ Cf. L. Tarán, op. cit., nota 41.

${ }^{53}$ The route of Parmenides: A Study of Word, Image, and Argument in the Fragments, New Haven 1970, 22 nota 31 in cui si arguisce: "The text must [...] be considered corrupt. Yet if $\pi \alpha ́ v \tau$ ' $\alpha$ ó $\tau$ should count now as no more than an emendation, it is closer to the text than the only

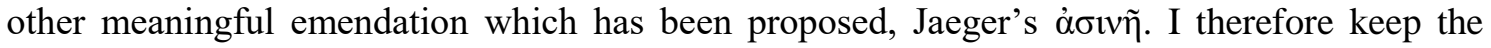
traditional reading", osservazioni ripetuta nella seconda edizione munita di una nuova introduzione, tre saggi aggiunti e un articolo inedito di Gregory Vlastos, "Names of Being in Parmenides", Las Vegas 2008.

${ }^{54}$ Parmenides: Die Fragmente. Munich: Heimeran 1974 [3rd ed. Zürich: Artemis und Winkle, 1995], 8. Cf. anche H. C. Günther, Aletheia und Doxa: das Proömium des Gedichts des 
Si riscontra per la prima volta la voce singolare di Tarrant che interpreta la lezione manoscritta 'A $\tau \eta$, lemma inteso come nome proprio riferito alla $\delta \alpha i ́ \mu \omega v$ e riferito sintatticamente come apposizione al relativo $\ddot{\eta}$, illustrazione molto problematica sotto tanti punti di vista, ma soprattutto per il fatto che non si producono indizi determinanti per convincere sull'ipotesi che si tratti della via 'rovinosa' per illusorietà causata da una divinità (malevola). ${ }^{55}$ Nonostante tale aspetto esegetico, che comunque ha una sua parziale logicità intrinseca - se qualcosa di negativo o di ostacolante o genericamente inappropriato non fosse capitato a proposito di tale via o non avesse in qualche modo interferito con essa, il valore e la scopo del poema parmenideo e soprattutto del suo proemio sarebbero quasi incomprensibili -, la proposta cadde in oblio per tutto il resto del secolo scorso, poiché una quasi invariata riproposta di 'A $\tau \eta$ ("Folly") con il significato di nome proprio di divinità per opera di Thayer è rimasta in qualche modo privata e non è dato precisare se Thayer sia solo idealmente $\mathrm{o}$ in qualche modo materialmente dipendente dall'intervento di Tarrant. Si narra che Thayer, interpellato in proposito, avrebbe risposto con l'ipotesi che tale sostantivo sia sintatticamente da

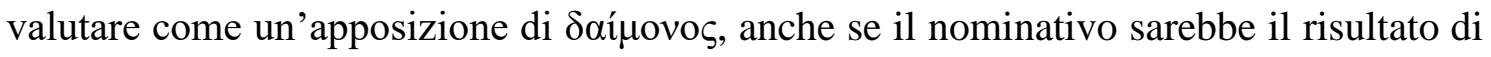
un fenomeno di attrazione del genitivo (del sostantivo di riferimento) in nominativo (dell'apposizione) all'interno di una proposizione relativa, quindi il nominativo ő́ varrebbe in effetti come un genitivo ö́n s. ${ }^{56}$

Parmenides, Berlin: Dunker und Humbolt (=Philosophische Schrifter 27), 1998, 13-14. A. Bernabé, De Tales a Demócrito. Fragmentos presocráticos, Madrid 1988/22001, 155.

${ }^{55}$ H. A. S. Tarrant, "Parmenides B.1.3: text, context and interpretation", Antichton 10, 1976, 17, il quale (escludendo altra morfologia con il dativo sia nella forma personificata "A quella di sostantivo generico örṇ, insostenibili per assenza di conforti filologici attendibili), commenta che Parmenide avrebbe inteso una via speciale in un primo stadio in cui si sarebbe trovato $(i b ., 4)$ e si sarebbe riferito alla divinità "identified with that power generally known as Atē", continuando a spiegare: "thus Atē would be one of the divinity's prosōpa, and stand here in apposition to the relative pronoun. Names of divinities tend to indicate functions in Parmenides' poem, and such a name would be related to her task of leading men of knowledge astray. Naturally the use of this name here would not exclude the use of the name Night for the same divinity later". Tuttavia, non pare che nel poema parmenideo si ravvisi che si metta in guardia da una simile (soprannaturale? numinosa?) potenza negativa oppure che qualcuno sia stato illuso o 'traviato' da una simile potenza. Del resto si annota in J. Palmer, Parmenides and Presocratic Philosophy, New York 2009, 377-378 che il giudizio di Terrant circa l'autenticità della lezione del cod. $\mathrm{N}$ "is in fact preferable to any previously conjectured alternative; but he

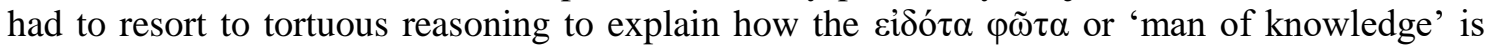
conveyed along a path of blind deception, and subsequent editors have properly ignored this proposal". Se la lezione è autentica, non può essere ricusata semplicemente perché qualche relativa spiegazione di un suo commentatore risulta tortuosa.

${ }^{56}$ La notizia è rilevata di seconda mano da Parmenides' Vision: A Study of Parmenides' Poem, University Press of America, 2016, 64 nota 2, in cui si riporta il seguente brano di un 
Autorevoli interpreti hanno mantenuto ancora di recente come più attendibile ő $\sigma \tau \eta$, che addirittura viene accolta come la lectio di N nell'edizione curata da A. Garcia Calvo. ${ }^{57}$ E. Renehan inoltre la giudica come "variant" e la spiega come la presunta corruzione con un'analisi paleografica ${ }^{58}$, piuttosto che l'autentico ö $\tau \eta$. Rincalza tale ipotesi J. Lesher con l'asserire persino che ö $\sigma \tau \eta$ "seems wholly appropriate in context". ${ }^{59}$ Il fatto che risulti (empiricamente?) la sua completa appropriatezza, non può essere motivo scientifico per assumere un simile giudizio e nemmeno ci possono rinvenire ragioni testuali o semantici che "Parmenides depicts himself - with good reason - as having advanced in his understanding far beyond any point previously

manoscritto datato "July 17, 1986": [...] your interpretation of the "way of the daimon" seems correct. Qu. 1 "can ó $\tau \eta$ be understood as in apposition to ódóv?" It can, but I would [be] inclined to take it in apposition with $\delta \alpha i$ í ovoc. Qu. 2 "Are there any precedents for a substantive with a relative clause being in apposition with antecedent?" Yes, Plato, Apology 41 a. Qu. 3 "could ơ $\tau \eta$ possibly be the name of the daimon would then be the antecedent to which ì refers?" Yes. Actually that is precisely what I think it is. Folly is that daimon. Had the third line read ö $\tau\rceil \varsigma$ instead of ö $\tau \eta$ this interpretation would have been immediately clear. A genitive or dative antecedent, when incorporated within a relative clause, is attracted in this fragment. I hope I have helped. Carl Thayer, S.J." Il parallelo platonico è abbastanza incomprensibile e non si dà ragione del motivo per cui il nome proprio di divinità (malevola) non si anticipi rispetto alla proposizione relativa. Ancora più significativamente non si riscontrano casi in cui il nome proprio di divinità sia associato al termine generico $\delta \alpha i ́ \mu \omega v$ o $\vartheta \varepsilon o ́ \varsigma(\mathrm{cf} . \mathrm{B} 1,22 ; \mathrm{B} 12,3)$, per cui sarebbe bastato impiegare il nome proprio 'A $\tau \eta$ come in $\mathrm{B} 1,14 ; \mathrm{B} 8,30$, osservazione che depone

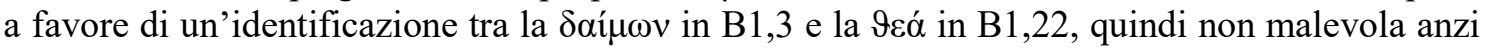
assolutamente benevola.

57 A. García Calvo, Lecturas Presocráticas I, Zamora 1981, 185; cf. anche D. SIDER in D. Sider-H. Johnstone, The Fragments of Parmenides, Bryn Mawr 1986, 8.

58 "[Rec.] The Fragments of Parmenides by A. E. Coxon", Ancient Philosophy 12: 2, 1992, 402, in cui si legge la seguente poco sensata osservazione: "if scholars had never been told, that ö $\sigma \tau \eta$ is a manuscript variant, there is little doubt, that someone would have long since proposed it independently by conjecture"; nemmeno ha molto senso una simile annotazione a riguardo: "Quod mihi dubium videtur, praesertim cum sciam inter plus quam viginti conjecturas, quas viri ingeniosissimi ac Graecae linguae scientia eminentissimi fecerunt, hanc abesse. Nihil vero lectio

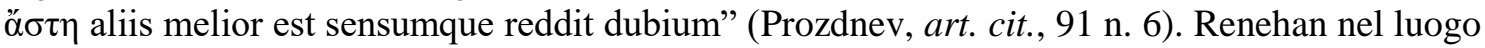
appena citato non solo non dimostra che sia corrotto il testo di N, ma adduce indizi paleografici che al contrario inducono a ritenere l'opposto. Il fatto che per lui si sarebbe verificato tra $\pi \tilde{\alpha} v$

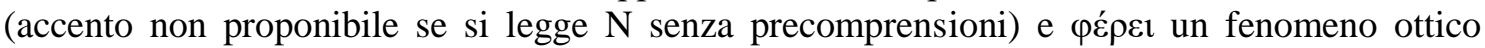
simile a $\tau \eta \alpha \tau$, significa solo che si sarebbe inserita una $\eta$ di troppo. Le sue valutazioni filologiche sono state formulate in risposta a quelle di H. Pelliccia, che vedeva nelle lettere $\tau \alpha \tau \eta$ introdotto per errore dello scrivano tra $\mathrm{i}$ due vocaboli per l'influenza del sintagma $\varphi \tilde{\omega} \tau \alpha \cdot \tau \tilde{n}$ фєро́ $\mu \eta v(\mathrm{~B} 1.3 \mathrm{~b}-4 \mathrm{a})$ in "The Text of Parmenides B 1,3 (D-K)", American Journal of Philology 109, 1988, 513-522, 511 (cf. per alcune osservazioni critiche anche se non propriamente soddisfacenti nonostante la sagacia encomiata in Prozdnev, art. cit., 98). Queste rilevazioni comunque confermano indirettamente che la lezione di $\mathrm{N}$ rimane l'unica accettabile, poiché $\tau \alpha \tau \eta$ è il tetragramma autentico effettivamente occorso tra i due suddetti vocaboli, anche se in verità $\pi \tilde{\alpha} v$ non può definirsi tale nel caso in esame.

59 “'The significance of $\kappa \alpha \tau \grave{\alpha} \pi \alpha \dot{v} \tau \tau^{\prime}$ ” $<\sigma>\tau \eta$ in Parmenides Fr. 1.3”, Ancient Philosophy 14, 1994, 1-20, 4 nota 4. 
reached by mortal men". ${ }^{60}$ Parmenide non viene ispirato dalla divinità, ma solo guidato

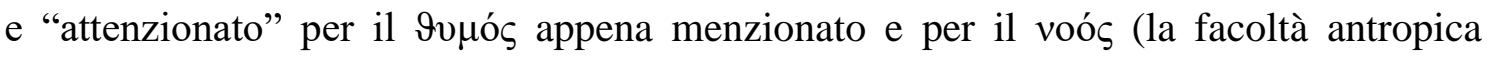
deputata a tale percorso cognitivo-concettuale), di cui egli è dotato (come ogni mortale). Continua una serie di commentatori che in modo più o meno convinto portano avanti questa linea ermeneutica. ${ }^{61}$ Una critica di un certo spessore dal punto di vista linguistico consiste nell' atticismo della forma ó $\sigma \tau \eta$, estremamente improbabile in epica ${ }^{62}$, per cui

${ }^{60} \mathrm{Ib}$., 16. L'analogia con Empedocle non funziona, perché Parmenide non è il destinatario di una rivelazione da diffondere attraverso la sua funzione di 'vate' divinamente abilitata nella via che porterebbe di città in città secondo il parallelo intravisto nella sesta Olimpiade di Pindaro, come in qualche modo si premetteva $(i b ., 8)$. Come si può percorrere la via che porta al sapere $\mathrm{e}$ rimanere sostanzialmente nell'ignoranza $o$ in una conoscenza incompiuta o quasi contraddittoriamente falsificante?

${ }^{61}$ R. McKirahan, Philosophy before Socrates. An Introduction with texts and commentary, Indianapolis ${ }^{1} 1994,151$. Nella seconda edizione $(2010,145)$ traduce: "which [or, "who"] brings a knowing mortal to all cities one by one", annotando nella nota 2 solo l'ambiguità di riferimento del pronome. Si legge in A. Laks, "Phénomènes et références : éléments pour une réflexion sur la rationalisation de l'irrationnel", Methodos, 2003, §3, 5 [Online] 3. URL: http://methodos.revues.org/205: "à travers villes" (caduta del tutto la menzione di $\pi \alpha ́ v \tau$ '); e nella relativa nota 17 si avverte: "Texte incertain. Pour le texte ici retenu, voir Lesher 1994". Si traduce "por todas as cidades" in J. Cavalcante de Souza, "Parmênides de Eléia. B-Fragmentos", in Os Pensadores Pré-Socráticos. Fragmentos, Doxographia e Commentários I, São Paulo 1996, 227 e, a parte la posizione nella frase, in J. T. Santos, Da Natureza. Parmênides. Lisboa, Alda, 1997 (São Paulo, Loyola, ${ }^{2} 2002$ ), 56-57, specificando che "percurso realizado será aquele que o sol descreve durante a noite"; "dans toute ville" si trova in J. Bollack, Parménide, de l'Etant au Monde, Lagrasse 2006, ad loc., credendo che si tratti del mondo che si sta per lasciare. Cf. anche J. Palmer, op. cit., 378, in cui conclude la sua disamina (in alcuni punti ondivaga) con questi saggi termini: "fortunately, not much depends on these lost words: while the crux presents a challenge for textual criticism, its remaining a crux hardly presents an obstacle to proper understanding of Parmenides' philosophy". Sfortunatamente per noi la crux riserva comunque elementi essenziali (non trascurabili) per la comprensione del pensiero e della composizione proemiale di Parmenide, come dimostra un così tanto affannarsi esegetico intorno ad esso. Nonostante questa pregevole prudenza, ci si arrischia ancora a sostenere: "the principles of textual criticism and paleography lend support to reading $\alpha<<\sigma>\tau \eta$ " e "[t]hus both literary parallels and philological craft underpin that emendation", concludendo con l'invito ad accettare "that $\alpha<\sigma>\tau \eta$ is most likely the correct reading" (M. Cosgrove, "The unknown 'knowing man': Parmenides B1.3”, The Classical Quarterly 61 (1), 2011, 28-47, 43-44), dopo aver accolte le tesi filologiche di Renehan ritenute sufficienti a dissolvere l'obiezione principale di Pelliccia (ib. 43), come già su rievocato. Il tutto per motivare l'indimostrata tesi che "the $\varphi \grave{s} s$

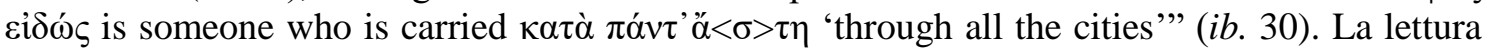
di $\ddot{\alpha}\langle\sigma\rangle \tau \eta$ viene accolta da Walter Ferrari in "Il sapiente e le città. Parmenide e una congettura involontaria su B1.3", in Testi frammentari e critica del testo. Problemi di filologia filosofica greca, 37, Roma 2013, 73-74 e in A. Laks-G. W. Most, Early Greek Philosophy, V: Western Thinkers, Part 2, "Parmenides", Cambridge-London 2016, 34, versione francese: Les débuts de la philosophie, cap. 19, "Parménide", Paris, 546.

${ }^{62}$ Cf. le osservazioni in E. Hülsz - B. Berruecos, "Parménides B1.3: una nueva enmienda", in ódoì voñoal Ways to Think. Essays in Honor of Néstor-Luis Cordero. Edited by Massimo Pulpito and Pilar Spangenberg, Bologna 2016, 31-61, 44, in cui si citano i due casi eccezionali: Eurip. Suppl. 952 e Pind. Neem. X, 5. 
F. Ferrari è propenso a modificarla con la forma non contratta $\alpha ́ \sigma \tau \varepsilon \alpha^{63}$, che dovrebbe valere come un errore imbastito su un altro errore. D'altro canto, anche dal punto di

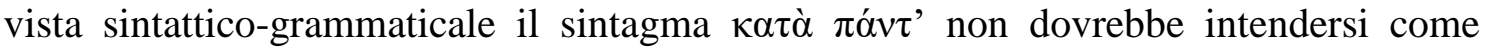
complemento di moto riferito a $\varphi \varepsilon ́ p \varepsilon ı v$. Questo verbo non si trova mai congiunto con

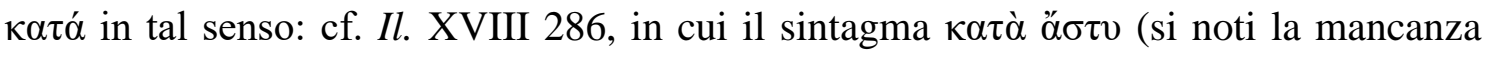
d'elisione della vocale finale della preposizione nel verso omerico per ragioni metriche, necessaria per queste stesse ragioni in quello parallelo parmenideo), circoscrive il luogo

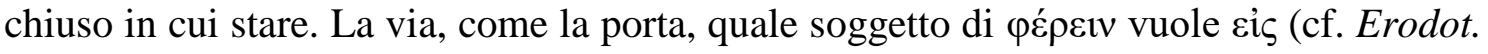
2, 122. 138; 9, 69; Eschil. Fr. 239; Tucid. 3,24) e più raramente غ̇ंí. ${ }^{64}$ Ferrari invoca diversi testi letterari a sostegno della ricostruzione del verso parmenideo con ó $\sigma \tau \varepsilon \alpha$,

${ }^{63}$ Il migliore dei mondi impossibili. Parmenide e il cosmo dei Presocratici, Roma 2010, 148 e si spiega nel commento: "Se infatti è stato dimostrato che la lezione astê, attribuita un tempo al codice N di Sesto Empirico (I.III) e poi accolta da Diels-Kranz, è solo il risultato di un errore di lettura, essa (o piuttosto astea con sinizesi di $-e a$ ) può tuttora rappresentare, in qualità di emendamento, una soluzione plausibile, accolta da Mourelatos 1970: 22 n. 31 e Lesher 1994, e può contare sul confronto con una serie di passi dell'Odissea omerica a partire da I.3". La sinizesi o sineresi non pare possibile per il lemma in questione (cf. le valutazioni e i riscontri a riguardo in Hülsz - Berruecos, op. cit., 44 e nota 65. Inoltre lo stesso Lescher riconosce: "the links between 1.3 and $O d$. xv 82 are tenuous at best" (op. cit., 1944, 4). Sagacemente Hülsz -

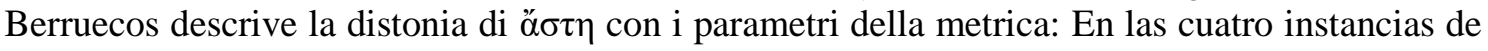
ó $\sigma \tau \varepsilon \alpha$ en Homero, la desinencia $\varepsilon \alpha$ está en la parte débil del pie (tesis), de manera que siempre es escanciada como dáctilo, mientras que en Parménides 1.3 la segunda sílaba de ǒ $\sigma \tau \eta$ quedaría en la parte fuerte (arsis) del tercer pie del verso" (op. cit., 44). Inoltre aveva già fatto problema l'atticismo di ö $\sigma \tau \eta$, per la cui discussione un po' speciosa si rinvia a Prozdnev, art. cit., 92 e note 11-14.

${ }^{64}$ Non è ammissibile l'accezione "supra omnes urbes" attribuita da Diels-Kranz sulla base di Il. XIX, 93 e da W. Guthrie in op. cit. II, 7, addirittura con l'interpretazione di $\delta \alpha i ́ \mu \omega v$ come maschile e allusivo del dio sole ricavato dalla dubbia congettura del presunto parallelo della già esaminata lamella di Turii (Orph. Fr., 42.7 Kern), come anche Guthrie riconosce e annota lo stesso H. Diels in "Ein orphischer Demeterhymnus", Festschrift Th. Gomperz, Wien 1902, 12. 15 (cf. la stessa perplessità in Lesher, op. cit., 14 nota 24). Nemmeno il senso è plausibile in

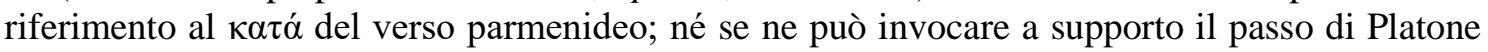

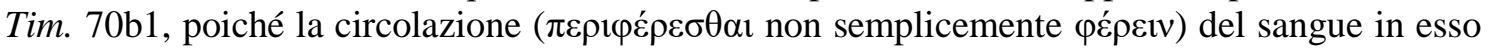
descritta potrebbe essere considerata relativa alla misura corrispondente ( $\kappa \alpha \tau \alpha$ ) a tutte le membra insieme. Inoltre, ammessa la valutazione analogica con il passo parmenideo, l'attestazione non è sufficiente da sola a giustificare una simile interpretazione. Arbitraria è l'immagine di Parmenide quale sapiente o vate che al pari di un rapsodo omerico o orfico va a insegnare per tutte le città, per cui la via sarebbe quella erronea e doxastica dei mortali (cf. J. Burnet, Early Greek Philosophy, London $\left.{ }^{4} 1930\right) 172$ n. 1; Parmenide. Testimonianze e Frammenti. Intr., trad. e comm. a cura di M. Untersteiner, Firenze ${ }^{2} 1979$, LII; H. Fränkel, "Parmenidesstudien", in Wege und Formenfrühgriechischen Denkens, München 1955, 156 Anm. 2; K. Riezler, Parmenides, Frankfurt a. M. 1934) 26). Non si ha traccia di nessun (auto)biografismo di tale genere concernente il destinatario della verità piuttosto che iniziato, come nemmeno che egli possegga prima una qualche sapienza. Il sapere umano ha una sua epistemica valenza immancabile solo se riferito alla verità e delucidato all'interno del quadro gnoseologico di essa. 
operazione di dubbio valore a questo proposito, dopo avere elaborato la descrizione dell'abusata formula epigrafica del Sole contenuta nella lamella di Turii ${ }^{65}$, ritenendo la congettura del Diels migliore tra le varie ipotizzate nonostante le citate obiezioni di Bernabé. Una simile valutazione risulta del tutto insostenibile per essere stata già ampiamente e ragionevolmente dimostrata falsa. ${ }^{66}$ Concesso quanto di per sé non ammissibile, il percorso dell'astro per tutte le città (o luoghi) non avrebbe nulla a che vedere con la conoscenza umana della verità, qualsiasi cosa questa espressione significhi, perché tale conoscenza implica un essere portati, come si ribadisce all'esordio in B1,4.

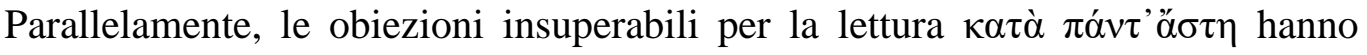
fatto fiorire una serie di reazioni filologico-ermeneutiche con varianti, alcune inedite e altre già avanzate in precedenza con o senza modifiche rieditate, che ugualmente soffrono di riscontri testicologici labili e di seconda mano, successivi al momento in cui

${ }^{65}$ Ferrari, op. cit., 158 nota 20, in cui si asserisce per il verso della lamina: "[...] il testo [...] è estremamente problematico", la sequenza delle lettere in questione benché non accolta del tutto

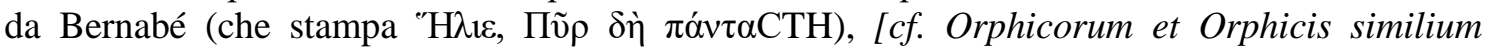
testimonia et fragmenta. Poetae Epici Graeci. Pars II. Fasc. 1. Bibliotheca Teubneriana. München-Leipzig 2004] è fra le meno problematiche del pezzo ed è stata recepita, dopo Diels e Zuntz, anche da Pugliese Carratelli 2005". In effetti, da autopsia condotta sulla foto della scritta presente nell'edizione di Kern (Orphicorum fragmenta, cit. 117), la sequenza indiziata non pare corrispondente alla effettiva, graficamente rilevabile, che risulta per il resto della linea 2 dopo

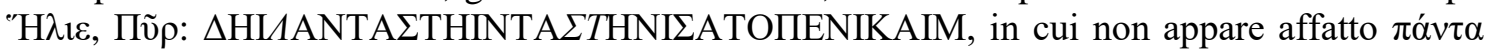
(le lettere poste in corsivo non sono certe, che potrebbero essere rispettivamente $\mathrm{X}, \mathrm{Z}$ e $\Gamma$ ). A parte tale appunto epigrafico, è da chiedersi: se il testo è "estremamente problematico", come riconosciuto da Ferrari, in che modo può ricavarsi una sequenza "meno problematica" e poi in che senso meno problematica, forse rispetto ad altre interpretazioni che non sono coinvolte con la lettura erronea del presunto parallelo parmenideo? L'elencazione dei passi letterari simili in cui si descrivono viaggi celestiali di eroi con "carro alato", assimilato al carro delle "Eliadi" che porta Parmenide (veramente appartiene alle Eliadi), non è molto rappresentativa, anzi magari va addotta per una direzione opposta. L'inno a Iside di Mesomede di Creta (nr. 5, Heitsch), in cui "la dea egizia è invocata, oltre che da molte altre entità, dagli astea diphrêlata (v.17), cioè da tutte le città attraverso le quali l'eroe eleusino Trittolemo è giunto sul carro alato di Demetra portando i doni dell'agricoltura", o Aristea di Proconneso (Herodot. IV, 13-15), Abaris iperboreo (Herodot. IV, 36), ecc., non hanno nulla di gnoseologico in senso proprio o parmenideo e tanto meno di definitivo e definitorio, come va valutata la via che $\varphi$ é $\varepsilon \varepsilon$ ("porta") alla stessa stregua in cui portava l'unico suo viandante mortale, senza alcun reale avvio e senza alcun reale ritorno, anche se di tale 'viaggio' vi sono delle 'contrazioni' o 'applicazioni' antropologiche peculiari. Non si tratta insomma di narrazioni singolari di fenomeni 'individuali' o storicamente delimitabili senza alcuna nota di universalità.

${ }^{66}$ Non si può non accogliere l'obiezione di A. Bernabé in "El fragmento órfico 47 Kern: ¿Texto mágico o sopa de letras?", in J. Peláez (ed.), El dios que hechiza y encanta. Magia y astrología en el mundo clásico y helenístico, Córdoba 2002, 55-71, 55, già prima avanzata da Pelliccia (op. cit., 508-10, cf. anche Prozdnev, art. cit., 92 nota 8) e rincalzata da Hülsz - Berruecos, art. cit., 45 e nota 48 (cf. anche Apéndice 2, 52). 
si è insinuata (erroneamente) la corruttela. La prima delle varianti inedite s'incontra

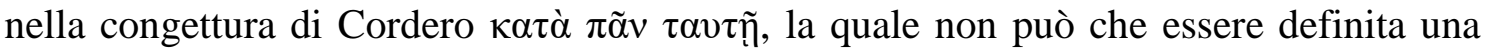
lettura emendativa sostanzialmente arbitraria, ricavata dal manoscritto A (Parisinus

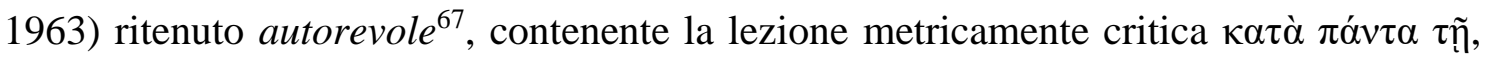
senza contare che la vocale $v$ che precederebbe $\tau$ ñ (i cui accenti o grafie sono chiare forme emendative arbitrarie) è frutto di pura immaginazione di alcuni critici. Segue subito dopo, tra quelle già edite, la congettura Meineke-Jaeger ( $\pi \dot{\alpha} v \tau^{\prime} \dot{\alpha} \sigma ı v \tilde{\eta}$, tradotto "unscathed"). ${ }^{68}$ Nonostante che $\pi \tilde{\alpha} v$ (anche per l'accento) non si riscontra affatto, esso è riproposto con la nuova versione prima inedita alquanto creativa e parzialmente assurda

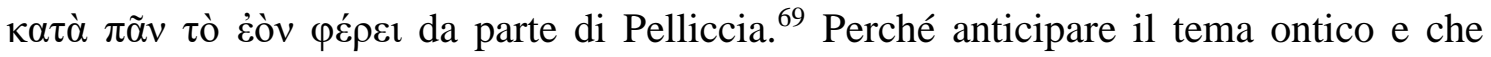
senso conferire proprio a $\pi \tilde{\alpha} v$ che suona mera ipotesi e nel contesto assolutamente pleonastico? Sulla stessa linea d'onda anche se non in modo coincidente, è la formula $\kappa \alpha \tau \grave{\alpha} \pi \alpha^{\prime} v \theta^{\prime}$ ö $\tau^{\prime}$ ẹ̌ avanzata da G. Cerri, benché infarcita di osservazioni anche acute

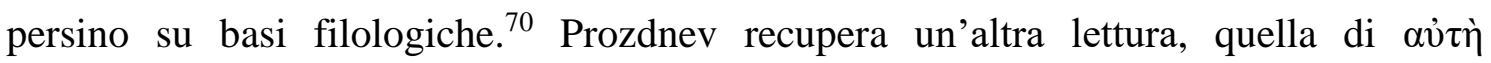
proveniente da Hermann ${ }^{71}$, che è priva di contenuti poco verificabili. Intanto una voce più unica che rara si è levata per ricordare la proposta "A $\tau \eta$ di Terrant basata

67 "Le vers 1.3 de Parménide ("La déesse conduit à l'égard de tout") », Revue Philosophique de la France et de l'Étranger 172, Études de philosophie ancienne: Hommage à Pierre-Maxime Schuhl, 1982,159-179, 171. Si crede che il manoscritto appartenga al cod. C (Cicensis 70), che avrebbe origine indipendente, e ipoteticamente in una serie di manoscritti, i cui copisti non avrebbero trascritto lo iota sottoscritto, ovvero Z (Paris. 2081), H (Vesontinus 409), Paris. 1965 e Paris. sup. 133. Tale tesi fu accettata da M. Conche in op. cit., 41 con la traduzione "à l'égard de tout <ce qu'il ya> (p. 42) e da Bernabé in Parménides. Fragmentos y tradición textual, con J. Pérez Tudela y N.L. Cordero, Madrid 2007, 20-21.

${ }^{68}$ Parmenides of Elea. Fragments, Toronto 1984, ad. loc.

${ }^{69}$ Op. cit., 1988.

70 "Il v. 1.3 di Parmenide: la ricognizione dell'esperienza," in Mousa, Scritti in onore di Giuseppe Morelli, Bologna 1997, 57-63.

${ }^{71} O$. cit., 99ss. La rievocata ragione paleografica intravista da Hermann concernente la frequente corruzione di $\alpha v$ per la legatura, non ha alcun fondamento autoptico per quanto riguarda il $\operatorname{cod}$. $\mathrm{N}$ (stante alla foto riprodotta in Coxon, op. cit., 2009, 42), almeno che la corruzione non si supponga avvenuta in precedenza. Il contrario è solo una malcelata mancanza di piena convinzione se si conclude: "Paleographicis rationibus, Parmenidis usui, poematis contextui omnium optime convenit lectio av̀ìे. Ergo Hermanni coniectura accepta, recte applicato vocabulo redditaque ei vi propria ad Parmenidis sententiam genuinam nos ascendere spero: "facunda deae via, quae eadem per omnia fert sapientem virum" (p. 101). Del resto, O. Primavesi annota: "la reproduction photographique du folio 124 verso de ce manuscrit, que l'on

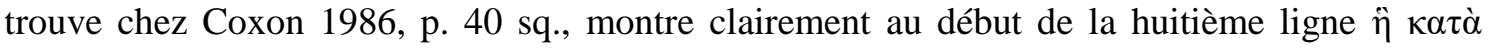
$\pi \alpha ́ v \tau$ ' ö $\tau$; voir Burkert 1969, p. 5-6 (= 5) ["Das Proömium des Parmenides und die Katabasis des Pythagoras", Phronesis, 14, 1-30 (= Burkert in Kleine Schriften VIII - Philosophica edd. Th. A. Szlezák \& K.-H. Stanzel, Göttingen 2008 (Hypomnemata, Supplement, 2) 1-27], n. 14, et Coxon 1986, p. 158.", 51 nota 39. 
sull'evidenza filologica del cod. $\mathrm{N}$ ma difesa nella opzione (meno graficamente giustificabile) di ö $\tau \eta$ da parte di J. Newell (2002) $)^{72}$, traducendo "con locura" o "en estado de confusión”. Al di là di tutto si legge la seguente notevolissima valutazione a riguardo: "En cualquier caso, la lectura de Tarrant-Newell no carece de significado, y se ajusta a la métrica"73, ma non è esatta o completamente provata per la morfologia e semantica.

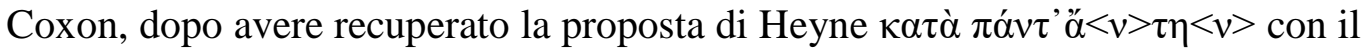
significato di "straight onwards" nella prima edizione, come già menzionato ${ }^{74}$, la rilancia con una variazione semantica nella seconda edizione con la seguente traduzione: "through every stage to meet her face to face a man of understanding". 75 Secondo la notizia indiretta, di recente (2012) A. P. D. Mourelatos, cambiando la sua precedente assunzione del ritenuto tradizionale $\pi \alpha^{\prime} \nu \tau^{\prime} \not \ddot{\alpha} \sigma \tau \eta$, ha formulato la congettura $\kappa \alpha \tau \grave{\alpha} \pi \alpha^{\prime} v \tau^{\prime} \quad \alpha<\dot{v}>\tau \grave{\eta}<v>$, che è rimasta inedita. ${ }^{76}$ Tuttavia Stuart B. Martin ${ }^{77}$ ripristina e

${ }^{72}$ Notizia ricavata da Hülsz - Berruecos, art. cit., 40 nota 41 concernente la tesi dottorale inedita Parmenidean Irony. Si anticipa l'ermeneusi ironica che si sviluppa in Cosgrove già rammentato.

${ }^{73}$ Ibidem.

${ }^{74}$ Op. cit., 69.

75 The Fragments of Parmenides. A Critical Text with Introduction, Translation, the Ancient Testimonia and a Commentary, edited with new translations by Richard McKirahan. With a new preface by Malcolm Schofield, Las Vegas 2009, 48. 49. Nel commento si spiega con una buona dose di immaginazione, nemmeno tanto simulata, anche se non può non essere accettata

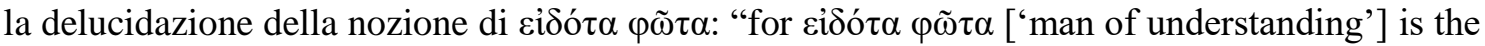

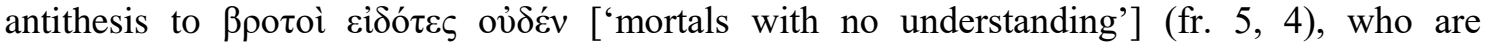

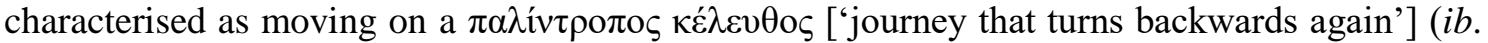
9), and in lines immediately following a passage of Homer to which P.[armenides] alludes in his description of the gateway (fr. 1, 14-21 after Il. $\Theta 393-396=$ E 749-752, see Introd. Sect. 3 (i)), the antithesis of $\pi \alpha \dot{\lambda} \lambda v \tau \rho \varepsilon \dot{\pi} \pi \varepsilon \sigma \theta \alpha 1$ ['turn backwards'] is ö $v \tau \eta v$ है $\rho \chi \varepsilon \sigma \theta \alpha 1$ ['meet face to face']

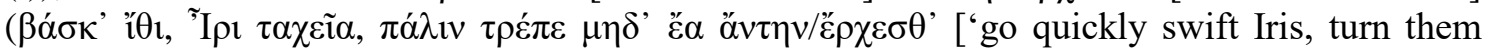
back and do not let them meet me face to face'], $\Theta$ 399-400). The correction ö $v \tau \eta v$ ['face to face'] is therefore exactly apt. It is not incompatible with the paraphrase cited by Sextus غ̇лì $\tau \grave{\eta} v$

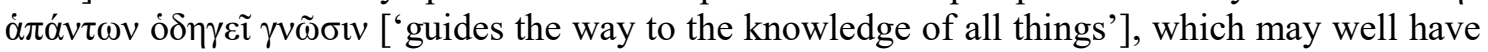
been made from an uncorrupted text." (ib., 271-272). Accolgono tale ricostruzione: W. Graham, The Texts of Early Greek Philosophy: The Complete Fragments and Selected Testimonies of the Major Presocratics I, Cambridge 2010, 210, benché nel commentario si ricordi la prima versione di Coxon "straight onwards" (ib., 235); J. Pòrtulas-S. Grau, "Parmènides de Vèlia", in id., Saviesa grega arcaica, Barcelona 2011, 677-769, 679-680; Primavesi, art. cit., 51.

${ }^{76}$ La notizia si legge in Hülsz - Berruecos, op. cit., 40 secondo il quale sarebbe stata esposta nella presentazione Disorientation, Hyperbaton, and Katabasis in Parmenides B1, in occasione del terzo congresso biennale della Asociación Internacional de Estudios Presocráticos presso Mérida, Yucatán (Messico) il 9 gennaio 2012. Si asserisce che Mourelatos adotterebbe una

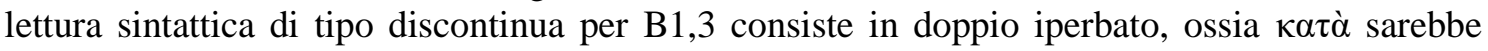

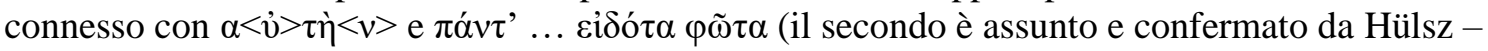


Berruecos) se si traduce: "of the goddess, who conveys along that very route every man who has gained knowledge" (ib., 49).

${ }^{77}$ Op. cit., 63. A. Lebedev, mostrandosi ignaro di quanto rilevato da Martin, propone $<\pi \mathrm{o}>\tau \tilde{\eta} 1$, una congettura davvero originale e curiosa, traducendo il verso: "[the road] of goddess who carries the man of knowledge by flight across the Universe [lit. «all things»]" (cf. "Parmenides,

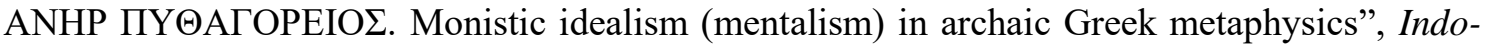
European Linguistics and Classical Philology XXI, Proceedings of the 21st Conference in Memory of Professor Joseph M. Tronsky, June 26-28 2017, 493-536), richiamando le immagini di viaggi alati in $O d$. V, 337, Arato, Inno a Merc. 544 e menzionando la similitudine giudicata "striking" del carro parmenideo presunto alato e l'anima con vं Platone (Fedro 246a3-b4). Si chiude la descrizione con la seguente conclusione: "The connection was recognized already by Hermias, In Platonis Phaedrum 122, $19 \mathrm{ff}$., and by some modern scholars, Hermann Diels and Paul Natorp among others." (in nota 12 si aggiunge Palmer 1999, 18). L'intervento su questa questione parmenidea potrebbe risalire a una bozza della presentazione, che Lebedev asserisce di avere esposto alla quarta conferenza biennale dell'International Association for Presocratic Studies (IAPS) tenuta all'Università Aristotelica di Tessalonica 30 giugno - 4 luglio 2014 (ib., 493 nota 1). Al di là di tale analogia abbastanza dubbia o almeno molto tenue, il proemio parmenideo non usa mai l'immagine del volo, ma solo del traino su una carreggiata, addirittura anche quando si tratta di attraversare il $\chi \alpha \sigma \mu$ ' $\alpha \chi \alpha v \varepsilon^{\prime} \varsigma$

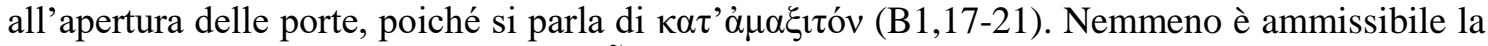

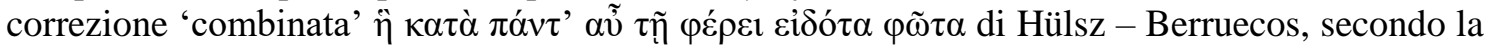
traduzione "el cual [camino] lleva de vuelta nuevamente ahí al hombre que sabe todas las cosas". Al di là della assunzione della prospettiva critica unanime su "DK28 B1.3, un verso que nos ha llegado corrupto" (op. cit., 32) senza una minima ombra di dubbio, e al di là della non riscontrata variante $\pi \alpha v^{\prime} \tau^{\prime}$ 'ó $\tau \eta$, inserita nella sequenza delle varianti riportate in parentesi senza connetterle con i relativi codici ( $i b ., 38)$, si parte da un ipotetico originale recante ПANTAYTH, che armonizzerebbe la "versión de Hermann, la reconstrucción de Mullach y la corrección de Diels, a pesar de sus diferencias", per poi aggiungere la versione di Cordero che "añadiría razonablemente una iota adscrita: ПANTAYTHI", ma da leggere diversamente, ossia due parole in luogo di una $(i b ., 47)$. Poco importa che "la propuesta tiene a su favor el hecho de que es mínimamente invasiva, cabe bien en el dialecto homérico, se ajusta al metro y da muy buen sentido en el contexto, como intentaremos mostrar" (ibidem). Il punto insuperabile è filologico: non si riscontra una dizione manoscritta quale AYTH (al di là delle altre determinazioni grafiche o ortografiche). Inoltre non è assolutamente necessario l'avverbio $\alpha \tilde{u}$ ("otra vez", "de nuevo", "de vuelta") per significare le seguenti funzioni attribuite a esso: "reitera la imagen del camino y el relato del viaje ya introducidos en los versos 1 y 2 , y señala la forma anular de la composición" (ibidem). La reiterazione è già palesemente impressa nella narratività della descrizione. Non si comprende nemmeno perché "otra vez" dovrebbe allude alla "forma anular" e invece non dovrebbe soddisfare in proposito uno stesso unico inizio e una stessa unica fine, visto che con la detta correzione si otterrebbe che "B1.3 mira prolépticamente a B5: $\xi$ vò̀v $\delta \dot{\varepsilon}$

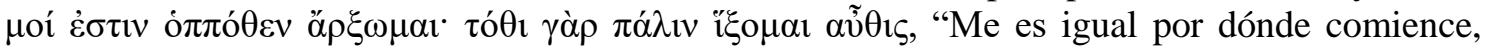
pues volveré allí nuevamente otra vez". Lo Govòv non permette una simile equipollenza con $\pi \alpha ́ \lambda \imath v$, poiché si tratta di sferica coincidenza della fine con l'inizio (non di una ripetizione del percorso dalla sua fine e quindi un ricominciare dal suo inizio dopo essere arrivati alla sua fine), ma arrivare alla sua fine significa essere di nuovo al suo cominciamento. Insomma s'insiste sempre e solo sulla sua unicità assoluta in qualunque estremo ci si trovi. Inoltre sarebbe stato

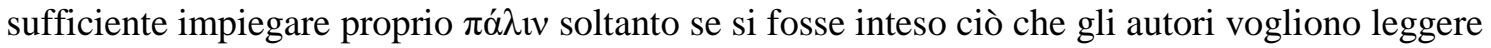
poiché non si parla d'inizio e fine del viaggio come in B5, ma dell'essenza della via stessa. Inoltre la lettura di un iperbato, ricavata dalle varie analisi combinate di Hermann/Mullach/

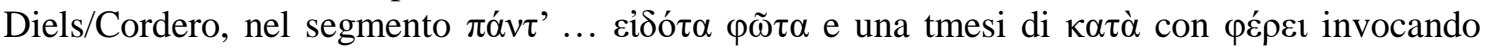
esempi omerici (Il. XXII, 354 e $O d$. III, 315-316) in modo da dare l'idea di un ritorno sulla stessa via da percorrere per l'uomo che sa tutto, risulta alquanto acuta ma al contempo poco confacente al contesto. Il tema è la modalità e la tipologica unica e incomparabile della via, non 
riconferma ớ $\rceil \eta$ di $\mathrm{N}$ che anche nella sua sintassi più limpida e inequivocabile di nominativo risulta la più accreditata e accreditabile lezione testuale, rilevata da TarrantThayer (non si cita Newell). In tal modo tale versione da definirsi come originale, nonostante qualche sporadico riconoscimento purtroppo abbandonato a sé stesso, non è stata del tutto obliata. Martin nel commento ricade però nelle inconcludenze esegetiche peculiari e contestuali, senza veramente decidere se si tratti di un nome personale o piuttosto del destino ruinoso di chi incorre in tale forza e senza veramente decidere se si

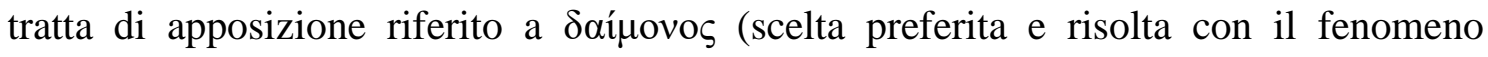
grammaticale dell'attrazione secondo l'analisi di Thayer, come ricordato in nota), oppure a óós, opzione definita "highly unlike" benché "grammatically possible" e soprattutto "to take impersonally".

Come si può essere sicuri che se si assume quest'ultima opzione non debba assolutamente trattarsi di un riferimento di nome proprio, ovvero che con essa non si intenda effettivamente la denominazione attribuita alla via o alla $\delta \alpha i ́ \mu \omega v$ ? Non si può non assumere che il lemma ö́ da valutarsi originale, continua a riservare in sé stesso gravi questioni ermeneutiche. Infatti, il senso più immediato potrebbe indicare il nome personale della $\delta \alpha i ́ \mu \omega v$ esiziale, di Sorte o Destino, sinonimo di Moĩ $\alpha$ o 'Epıv́́s, come in qualche modo chiarirebbe la dea in $\mathrm{B} 1,26$ senza o con attrazioni di casi come già discusso ${ }^{78}$. In tal modo si potrà valutare $\kappa \alpha \tau \grave{\alpha} \pi \alpha ́ v \tau(\alpha)$ non come neutro plurale (aggettivo o meno) ma come maschile singolare riferito a cíó́ $\tau \alpha$ $\varphi \tilde{\omega} \tau \alpha$. Ne conseguirebbe la seguente traduzione: "la quale, Sorte, porta (fa ottenere o giungere) contro ogni uomo che sa",

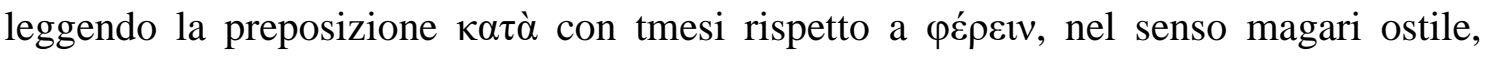
ovvero di portare giù, scaricare in modo da nascondere una valenza simbolica rinviante

la specificazione del sapere dell'uomo che, già va da sé, deve essere totale, altrimenti non si può conseguire appunto un sapere degno di tale nome o nella sua verità. L' "uomo che sa" non può non essere che riferito a sé stesso (magari con una figura retorica in terza persona generica) per garantire che quanto egli sta per raccontare non è altro che l'esposizione del viaggio e quindi le sue acquisizioni in termini gnoseologici. È la via che procura nella sua funzione di condurre chi in modo peculiare e singolare finalmente ha raggiunto la verità rispetto alla quale si rimane per sempre una volta approcciata. Ormai è da permanere nella via che porta il suo conoscitore senza più possibilità di smarrimento per le assicurazioni narrative proemiali (cosmo-teologiche?) ma soprattutto per le prove teoretiche inconfutabili offerte dalla dea che senza la via stessa non può svelare alcuna verità, nella quale la stessa divinità è da sempre collocata, e non potrebbe essere diversamente.

${ }^{78}$ Cf. de Ate. "Excursus I ad lib. XIX, (T) 90". Variae Lectiones et Observationes In Iliadem. Cvrante C. G. Heyne, V. I 2, 11. XV-XIX, Lipsiae-Londini MDCCCII, 704ss. 
all'intento di dimostrare illusorio il sapere umano, che appunto alla fine va eluso. Quest'aspetto non può essere del tutto espunto, poiché non si può testualmente escludere l'antitesi radicale tra il conoscere parmenideo e il (non) sapere umano che non può non interessare la $\delta \alpha i ́ \mu \omega v$ e quindi il tessuto tematico del poema sin dalle sue primissime battute. Nel proemio tale antitesi non è ancora tematizzata ma solo proletticamente si declina nel suo effetto a proposito della via, per sottolineare la condizione altrettanto essenziale benché di tipo esogeno, ovvero subita a seguito dell'universale comportamento improprio di chi la doveva percorrere e non l'ha percorsa per nulla. In altri termini, s'intende dire che la via e la sua polifemicità sono rimaste in qualche modo 'congelate' e ormai lasciate in forma d'irrintracciabilità.

$\mathrm{Si}$ potrebbe ancora interpretare il lemma in senso più generico che specificherebbe l'azione della $\delta \alpha i ́ \mu \omega v$ che appunto sarebbe vendetta (punizione) o vendicatrice (punitrice) in tutto o contro tutto ciò che ostacolerebbe il sapere umano, incluso l'universale stato di insipienza e erranza antropica, anticipando in qualche modo la qualifica della Dike in B1,14, nella quale quindi si dovrebbe anche identificare la $\delta \alpha i ́ \mu \omega v$. La via però non può appartenere a una simile $\delta \alpha i ́ \mu \omega v$, ma alla dea che svela la verità, anche se proprio una simile operazione richiede l'organizzazione del viaggio a suo tempo intrapreso dal kouros che non avrebbe molto senso senza coordinarlo a tale opposizione, in altre parole, senza la situazione universale antropica noeticamente avversa alla vera conoscenza oppure ormai in stato d'irreversibile incapacità alla vera conoscenza.

Tuttavia, per tale dettaglio non è necessario avvalersi di acrobazie sintattiche troppo sofisticate (per quanto lecite), come quelle proposte, soprattutto in caso in cui letture grammaticali più piane sono possibili anzi poziori. Più nitidamente, infatti, il

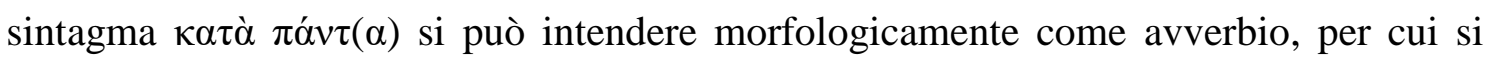
otterrebbe questa altra traduzione: "la quale, (essendo la dea) Sorte, porta in tutto (in assoluto) l'uomo che sa". Non sembra però soddisfacente, oltre al già evidenziato fattore stilistico che imporrebbe semplicemente il nome proprio senza quello generico (metricamente perfetto), sia perché la sorte divinizzata o meno non dovrebbe dotarsi della funzione di portare in riferimento al sapere umano, funzione che è propria magari delle cavalle o appunto di vie in genere. Inoltre il mero portare non potrebbe intendersi in una direzione ostile, come se fosse fuorviante, ma magari in un'accezione opposta, nel senso di favorire o causare il sapere che in sé stesso non può non essere positivo e autentico, non propriamente collegabile al sostantivo ö $\tau \eta$ o al nome 'A $\tau \eta$. In tal modo si 
dovrebbe immaginare un processo metamorfico altamente improbabile di stampo eschileo, che da negativa o devastante la Sorte si muti in una diametralmente opposta, positiva e costruttiva. Del resto, il sapere parmenideo dell'uomo che sa non può non essere quello vero, poiché le modalità di acquisizione, l'essere portato da dea o da via preposta proprio a tale fine, si sono descritti proprio per garantire l'infallibilità di tale sapere umanamente declinato e declinabile. Per il sapere non vero e per rimanervi, nessun intervento per un viaggio su una simile via avrebbe senso, se appunto la via non favorisse di accedere alla conoscenza indefettibile della verità. Tuttavia nulla di irrazionalmente fatale può reputarsi connesso con il viaggio come si chiarisce inequivocabilmente in B1,26-28, ma si tratta sempre di un tragitto legato alla

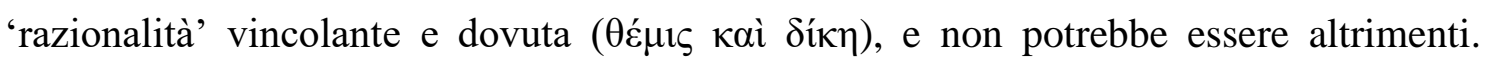
Inoltre il punto non è la specificazione della divinità che comunque rimane imprescindibile ma della via, specificazione altrettanto immancabile.

La sola denotazione di via polifemica (dal sentenziare irrevocabile) e della sua connessione divina non è sufficiente a determinare integralmente o compiutamente la via in questione, perché manca l'aspetto ineludibile che è necessariamente implicato nella definizione, in relazione alla suddetta situazione di umana intangibilità per essere stata inopinatamente disattesa a motivo della 'inammissibile' incongruenza intrapresa dai mortali - costoro per loro propria 'colpa' in tal modo l'hanno mancata in termini epistemici e si sono condannati al non sapere o a un sapere fittizio. L'unica forma metodologica per giungere al sapere, è stata 'rovinosamente' svuotata o resa indisponibile o genericamente inibita a motivo della deviazione umana, per cui si è rimasti in una universale fallacia inopinata. Il presente indicativo (a differenza del participio restituito per le cavalle, modo verbale che si ripresenta nel proemio solo per la descrizione delle $\pi v ́ \lambda \alpha \imath$ in $\mathrm{B} 1,11$ ) ha la nuance definitoria per tale via. La comprensione di B1,3 è in parte minata dalla presenza dell'espressione già citata ritenuta corrotta, ovvero $\pi \alpha ́ v \tau$ 'ó $\tau \eta$. Questa lezione è la più antica e riportata dall'unico codice che si è dimostrato in più casi il solo in regola per fare testo, perché copia inalterata della versione originale, e quindi ne va cercato il senso prima di dichiararla corrotta o finché non si trovano ragioni oggettive filologiche per assumere una tale posizione. In effetti, áı 
significato di "lesa" "79, che ha dato origine anche alla più usata forma sostantivata. Ciò vuol dire che questa via la sola che garantisce il sapere con il suo portarvi l'umanità, è stata lesa, compromessa, pregiudicata, violata nel suo intimo (disastrosamente disattesa) dai mortali che l'hanno 'colpevolmente' (contro la naturale disposizione) ignorata o da essa hanno completamente deviato, inficiando così del tutto la propria

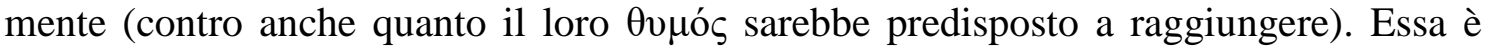
rimasta fuori dal loro presunto percorso gnoseologico, perché i mortali hanno intrapreso e continuano a intraprendere un percorso altro o apposto che pertanto è solo fittizio $(B 1,26)$ causando la propria infatuazione nefasta. Con questa 'catastrofica', 'disfunzionale', 'distopica' alternativa (pseudo)cognitiva si è realizzato, parallelamente, anche un trattamento ineluttabilmente erroneo che ha prodotto apparenze cognitive per le quali i mortali non si possono definire esseri umani che sanno (benché lo possano considerare e considerano altrettanto erroneamente), contenuto che, nonostante tutto, non può non riguardare ineluttabilmente ormai la via che appunto deve metodologicamente e teoricamente (indirettamente o meno) contrastare o dimostrare infondata e fallace anche la natura di tale contenuto umanamente elaborato. La condizione di errore umano pressoché universale non può non modificare metodologicamente la via, nemmeno esteriormente 'scalfirla', ma debitamente 'interessarla' e necessariamente 'conivolgerla' in modo elenchico. I travisamenti della verità hanno prodotto tuttavia la situazione di una sua ormai irreversibile impraticabilità o meglio 'pratica impraticatezza' dovuta all'antropica attitudine insciente verso la via stessa, nel senso che si è praticato ciò che impediva di praticarla in modo che si è distonicamente, indebitamente e indifferentemente ignorata. La via nella sua essenziale definizione rimane intatta, ma subisce una negativa o adulterante ritorsione nella sua operatività ed efficienza filosofica, nel senso che intorno a essa non si può non tenere conto di tale decisiva erroneità e dimostrare la 'vera' fisionomia di tale situazione

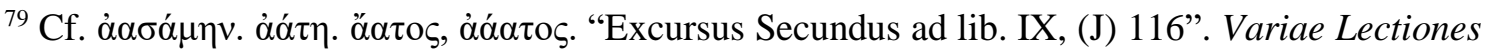
et Observationes in Iliadem. Curante C. G. Heyne, V. I 2, 11. V-IX, Lipsiae-Londini MDCCCII,

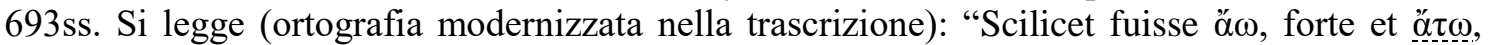

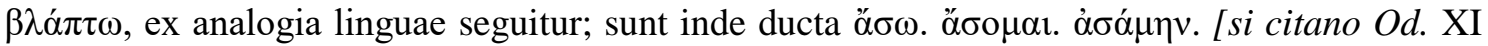

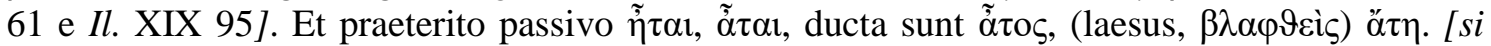
menzionano altri esempi greci].", in modo da potere evincere che si è formato un aggettivo (a tre uscite) $\tilde{\alpha} \tau o \varsigma, ~ \alpha ̈ \tau \eta, ~ \tilde{\alpha} \tau o v$, sinonimo di $\beta \lambda \alpha \varphi \vartheta \varepsilon i ́ \varsigma$, quasi mai attestato o per niente - proprio la crux parmenidea dovrebbe rappresentarne un'attestazione più unica che rara (perché la polisemia che riserverebbe creerebbe ambiguità di diverse omonimie, come anche l'appena citato "Excursus" evidenzia).
} 
negativa con la prova elenchica della sua inconsistente fenomenologia. In altre parole la via non può essere rimasta del tutto immune dalla 'lesione follemente subita', della 'violazione demente' che si è irrazionalmente ritorta contro di essa e ha provocato in essa e per essa un 'contraccolpo rovinoso' in termini epistemici che è rimasto inconscio e inavvertito nella mente degli uomini.

Questa lezione del manoscritto più antico, già da subito percepita corrotta tanto che tutti gli altri codici successivi non la rispettano e cercano di correggere o riportare goffe lezioni, invece non solo ha un senso innegabile, ma è l'unica che veramente dispiega uno degli aspetti essenziali della via a seguito del comportamento negativo intrapreso dai mortali verso di essa, che non può non essere proletticamente sotteso sin dai primi versi. Da essi è stata resa inaccessibile o non praticata in modo che essa da assoluta prospettiva di verità soggettivamente non è stata nemmeno percepita, notata. L'effetto di tale inettitudine consiste nel fatto che si è offuscata e pertanto 'si è rovinata', disattesa nella sua funzione gnoseologica, benché sarebbe stato necessario mettere in pratica esattamente il contrario, di percorrerla nella sua assoluta esclusività. I mortali smarrendola del tutto, nella loro consequenziale confusione non si sono resi conto di avere istituito o costruito soltanto 'un falso' o un che di 'falso' (B8,58ss), con cui l'hanno inscientemente scambiata o interamente esclusa. Parmenide con l'aggettivo ót $\rceil$ non proprio usuale intendeva evidenziare la difficoltà estrema, ormai avvertita e genericamente prospettata insormontabile che si è fatta risultare per tale via a causa della negligenza decettiva dei mortali, per cui al fine di giungere al suo traguardo è

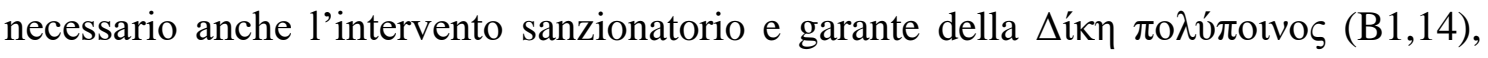
oltre all'equipaggiamento di agenti speciali per il viaggio. La condizione negativa che si è introdotta, non riguarda la bontà (polifemica) epistemologica della via in sé stessa, la cui autenticità è narrativamente anticipata nei primi versi del proemio, ma in relazione al sapere umano, per il quale essa rimane comunque assoluta conditio sine qua non.

Il sintagma $\kappa \alpha \tau \grave{\alpha} \pi \alpha ́ v \tau(\alpha)$ enfatizza la situazione negativa in senso olistico assumendo così una valenza avverbiale già rilevata recante il senso di in tutto, completamente, assolutamente per specificare la sua condizione di non essere praticata, come si doveva e si dovrebbe, per 'imperizia' (gnoseologica) degli uomini, condizione che rischia di determinare una impraticabilità irreversibile perché risulta ormai fatalmente (irremovibilmente) arrecata in termini di percezione o di oscuramento nei confronti di tale óoós. Questa via, ormai impervia perché misconosciuta in sé stessa e del tutto ostruita o impedita o semplicemente non battuta dagli uomini, è e rimane 
l'unica 'oggettivamente' funzionale in termini gnoseologici, è e rimane la sola che abilita con il suo percorso universalmente valido per la conoscenza del vero e quanto l'animo (quasi inconsciamente) vorrebbe raggiungere (e quello personale di Parmenide ha effettivamente raggiunto a suo tempo). Infatti, è proprio l'unica realmente percorribile in termini gnoseologici, essendo anche 'esclusiva' nel mediare per lo svelamento della verità; ma essa risulta completamente irriconoscibile o del tutto

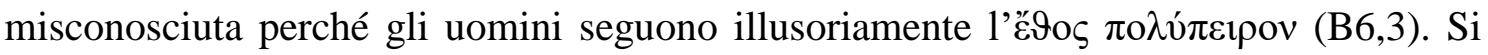
può approntare quindi la seguente traduzione di B1,1-4a:

Cavalle, e esse, portando me [Quando cavalle, e esse, portarono me], fin dove l'animo raggiungerebbe,

come quinto (con le quattro [presenti]), dopo che vennero a trasportami a (una) via magniloquente

di demone (femminile), la quale ( $s c$. via) in tutto 'alienata' (disattesa; occlusa) [dai mortali] porta uomo che sa,

vi ero portato (destinato [ad arrivare])...

Vittorio Ricci

Università Roma 2 - Tor Vergata

\section{Bibliografia}

AA. VV. (2007) Allegoristi dell'età classica. Opere e frammenti, Milano.

Albertelli, P. (1939) Gli Eleati. Testimonianze e frammenti, Bari.

Austin, S. (1986) Parmenides: Being, Bounds, and Logic. Yale University Press.

Azevedo, C. A. (2015) "Parmênides e o novo "mestre da verdade", Anais de Filosofia Clássica 9, $\mathrm{n}^{\mathrm{o}} 17: 61-74$.

Bekker, I. (1842) Sextus Empiricus, Berolini Typis et Impressis Gr. Reimeri.

Bergk, T. (1886 [1839]) "Commentatio de Empedoclis prooemio", Kleine philologische Schriften II, Halle.

Bernabé, A. (2001 [1988]) De Tales a Demócrito. Fragmentos presocráticos, Madrid.

Bernabé, A. (2002) “El fragmento órfico 47 Kern: ¿Texto mágico o sopa de letras?”, in J. Peláez (ed.), El dios que hechiza y encanta. Magia y astrología en el mundo clásico y helenístico, Córdoba: 55-71.

Bernabé, A. (2007) Parménides. Fragmentos y tradición textual, con J. Pérez Tudela y N.L. Cordero, Madrid. 
Böckh, A. (1836) Parmenidis libri de natura exordium emendatur, Berlin (ristampato in Gesammelte kleine Schriften, Leipzig, vol. IV).

Bollack, J. (2006) Parménide, de l'étant au monde, Paris.

Bowra, C. M. (1937) “The Proem of Parmenides”, Classical Philology 32.2: 97-112.

Brandis, A. (1813) Commentationum Eleaticorum pars prima, sectio secunda: de Parmenide, Altonae.

Burkert, W. (1969) "Das Proömium des Parmenides und die "Katabasis" des Pythagoras", Phronesis 14: 7-9.

Burnet, J. (1930) Early Greek Philosophy, London.

Cavalcante de Souza, J. (1996) "Parmênides de Eléia. B-Fragmentos", in Os Pensadores PréSocráticos. Fragmentos, Doxographia e Commentários I, São Paulo.

Cerri, G. (1997) "Il v. 1.3 di Parmenide: la ricognizione dell'esperienza," in Mousa, Scritti in onore di Giuseppe Morelli, Bologna: 57-63.

Cerri, G. (1999) Parmenide di Elea, Poema sulla natura, Milano.

Cerri, G. (1999) Parmenide di Elea. Poema sulla natura, Milano.

Clark, R. J. (1979) Catabasis: Virgil and the Wisdom-Tradition, Amsterdam.

Claus, D. B. (1981) Toward the Soul: An Inquiry into the Meaning of Psyche before Plato, New Haven, Yale University Press.

Condello, F. (2016) "Nuovi studi parmenidei tra filologia e dialettologia", Eikasmos. Quaderni Bolognesi di Filologia Classica XXVII: 495-519, 502-503.

Cordero, N. L. (1982) "Le vers 1.3 de Parménide ("La déesse conduit à l'égard de tout")", Revue Philosophique de la France et de l'Étranger 172, Études de philosophie ancienne : Hommage à Pierre-Maxime Schuhl: 159-179.

Cordero, N.-L. (2004) By Being, It Is: The Thesis of Parmenides. Parmenides Publishing.

Cornford, F. M. (1952) Principium sapientiae: The Origins of Greek Philosophical Thought, Cambridge University Press.

Cosgrove, M. (2011) “The unknown 'knowing man': Parmenides B1.3”, The Classical Quarterly 61 (1): 28-47.

Couloubaritsis, L. (1986) Mythe et philosophie chez Parménide, Bruxelles.

Coxon, A. H. (1968), “The text of Parmenides fr. 1,3”, Classical Quarterly, 18.

Coxon, A. H. (2009) The Fragments of Parmenides. A Critical Text with Introduction and Translation. The Ancient Testimonia and a Commentary, Las Vegas, Parmenides Publishing.

Curd, P. (2004) The Legacy of Parmenides. Eleatic Monism and Later Presocratic Thought, Las Vegas.

Cursaru, G. (2016) "Le Proème de Parménide : anabase et /ou catabase ?", Cahiers des études anciennes, LIII -1, 39-63. [on line] URL: http://etudesanciennes.revues.org/907.

De Vogel, C. (1950) Greek philosophy; a collection of texts with notes and explanations, vol. I, Leiden.

Deichgräber, K. (1958) Parmenides Auffahrt zur Göttin des Rechts. Untersuchungen zum Proömion seines Lehrgedichts, Wiesbaden Steiner.

Di Toro, U. (2010) L'enigma di Parmenide. Poesia e filosofia nel proemio, Roma.

Diels, H. (1897) Parmenides Lehrgedicht, Berlin 1897. 
Diels, H. (1901) Poetarum philosophorum fragmenta. Auctore Udalrico de WilamowitzMoellen-dorf Collecta et Edita, vol. III, Berlin 1901.

Diels, H. (1902) “Ein orphischer Demeterhymnus”, Festschrift Th. Gomperz, Wien.

Diels, H. (1912 [1903] [1906]) Die Fragmente der Vorsokratiker, Berlin.

Diels, H. (1929) Doxographi Graeci, Berolini-Lipsiae.

Dolin, E. F. (1962) "Parmenides and Hesiod", Harvard Studies in Classical Philology 66: 9398.

Drozden, A. (2001) "Parmenides' Theology", Eranos 94: 4-15

Eisler, R. (1910) Weltmantel und Himmelszelt, München.

Estienne, H. (1573) "Parmenidis Carmina", in Poesis Philosophica, Genève: 41-46.

Fabricius, A. (1718) Sexti Empirici Opera graece et latine, Leipzig.

Ferrari, F. (2010) Il migliore dei mondi impossibili. Parmenide e il cosmo dei Presocratici, Roma.

Fränkel, H. (1951) Dichtung und Philosophie des frühen Griechentums. Eine Geschichte der griechischen Epik, Lyrik und Prosa bis zur Mitte des fünften Jahrhunderts, New York.

Fränkel, H. (1955) "Parmenidesstudien", in Wege und Formenfrühgriechischen Denkens, München.

Fränkel, H. (1975) Early Greek Poetry and Philosophy, Oxford, Blackwell.

Fülleborn, G. G. (1795) Parmenidou Tou Eleatou Leipsana. Fragmente Des Parmenides. Gesammelt, Übersetzt Und Erläutert von Georg Gustav Fülleborn. Zûllichau.

Furley, D. J. (1989) Cosmic Problems: Essays on Greek and Roman Philosophy of Nature, Cambridge University Press.

Gallop, D. (1984) Parmenides of Elea: Fragments, University of Toronto Press.

García Calvo, A. (2008) Lecturas Presocráticas I, Zamora 1981.

Gemelli Marciano L. (2008) "Images and Experience: At the Roots of Parmenides' Aletheia", Ancient Philosophy 28: 21-48.

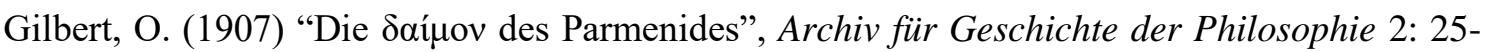
45.

Graham, W. (2010) The Texts of Early Greek Philosophy: The Complete Fragments and Selected Testimonies of the Major Presocratics I, Cambridge.

Gunther, H. C. (1998) Aletheia und Doxa: das Proömium des Gedichts des Parmenides, Berlin: Dunker und Humbolt (=Philosophische Schrifter 27).

Guthrie, W. K. C. (1965) A History of Greek Philosophy, vol. 2: The Presocratic Tradition from Parmenides to Democritus, Cambridge, Cambridge University Press.

Heitsch, E. (1995 [1974]) Parmenides: Die Fragmente. Munich: Heimeran.

Hermann, A. (2004) To Think Like God: Pythagoras and Parmenides. The Origins of Philosophy, Las Vegas, Parmenides Publishing 2004.

Hermann, J. G. J. (1827) Opuscula 1, Leipzig.

Heyne, G. (1802) "Excursus I ad lib. XIX, (T) 90". Variae Lectiones et Observationes in Iliadem, V. I 2, 1l. XV-XIX, Lipsiae-Londini, 704ss.

Hülsz, E. \& Berruecos B. (2016) "Parménides B1.3: una nueva enmienda", in ódoì voñoal Ways to Think. Essays in Honor of Néstor-Luis Cordero. Edited by Massimo Pulpito and Pilar Spangenberg, Bologna. 
Jaeger, W. (1936) Paideia, Berlin.

Karsten, S. (1835) Parmenidis Eleatae carminis reliquiae. De vita eius et studiis disseruit, fragmenta explicavit, philosophiam illustravit S. Karsten, Amsterdam.

Kern, O. (2004) Orphicorum et Orphicis similium testimonia et fragmenta. Poetae Epici Graeci. Pars II. Fasc. 1. Bibliotheca Teubneriana. München-Leipzig.

Kingsley, P. (1999) In the Dark Places of Wisdom, London.

Kirk, G. S. \& Raven, J. E. (1957) The Presocratic Philosophers, Cambridge.

Kirk, G. S.; Raven, J. E. \& Schofield, M. (2007) The Presocratic Philosophers, Cambridge'.

Kochalsky, A. (1911) De Sexti Empirici adversus Logicos libri quaestiones criticae, Marburg: 9-104.

Laks, A. \& Most, G. W. (2016) Early Greek Philosophy, V: Western Thinkers, Part 2, "Parmenides", Cambridge-London (Les débuts de la philosophie, cap. 19, "Parménide", Paris).

Laks, A. (2003) "Phénomènes et références : éléments pour une réflexion sur la rationalisation de l'irrationnel", Methodos [Online] URL: http://methodos.revues.org/205.

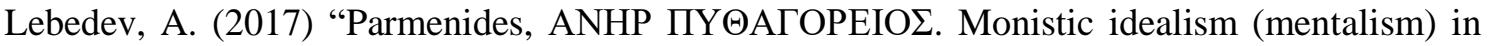
archaic Greek metaphysics", Indo-European Linguistics and Classical Philology 21, Proceedings of the 21st Conference in Memory of Professor Joseph M. Tronsky, June 26-28: 493-536.

Lee, E. N.; Mourelatos, A. P. D. \& Rorty R. M. (1973), "Exegesis and Argument: Studies in Greek philosophy presented to Gregory Vlastos", Phronesis (Supplementary Volume).

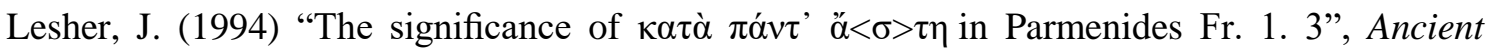
Philosophy 14: 1-20.

Lombardo, S. (1982) Parmenides and Empedocles. Grey Fox Press.

Mansfeld, J. (1964) Die Offenbarung des Parmenides und die menschliche Welt, Assen 1964.

Martin, J. H. (2003) Parmenides of Elea. A Verse Translation with Interpretative Essays and Commentary to the Text, Westport-Connecticut-London.

McKirahan, R. (1994) Philosophy before Socrates. An Introduction with texts and commentary, Indianapolis.

McKirahan, R. (2009) The Fragments of Parmenides. A Critical Text with Introduction, Translation, the Ancient Testimonia and a Commentary, with a new preface by Malcolm Schofield, Las Vegas.

Morgan, K. A. (2000) Myth and Philosophy from the Presocratics to Plato, Cambridge University Press.

Morrison, J. S. (1955) "Parmenides and Er", Journal of Hellenic Studies 75: 59.

Mourelatos, A. P. D. (1970) The Route of Parmenides, New Haven, Yale University Press.

Mullach, F. G. A. (1845) Aristotelis de Melisso, Xenophane, Gorgia disputationes, cum eleaticorum philosophorum fragmentis, Berlin.

Mullach, F. W. A. (1860) Fragmenta philosophorum Graecorum [vol. 1], Poeseos philosophicae caeterorumque ante Socratem philosophorum quae supersunt, Paris.

Mutschmann, H. (1914) Sexti Empirici Opera. II, Lipsiae.

Owens, J. (1979) "Knowledge and katabasis in Parmenides", The Monist 62: 15-29.

Palmer, J. (1999) Plato's Reception of Parmenides, Oxford University Press: Oxford.

Palmer, J. (2009) Parmenides and Presocratic Philosophy. Oxford University Press: Oxford. 
Patin, A. (1899) "Parmenides im Kampfe gegen Heraklit", Jahrbücher für classische Philologie Suppl. 25.

Pelliccia, H. (1988) "The Text of Parmenides B 1,3 (D-K)", American Journal of Philology 109: 513-522.

Pellikann-Engel, M. E. (1974) Hesiod and Parmenides: A New View on Their Cosmologies and on Parmenides' Proem, Amsterdam.

Pfeiffer, H. (1975) Die Stellung des parmenideischen Lehrgedichtes in der epischen Tradition, Bonn.

Pohlenz, M. (1937) Herodot. Der erste Geschichtschreiber des Abendlandes, Leipzig.

Pòrtulas, J. \& Grau, S., (2011) "Parmènides de Vèlia", in id., Saviesa grega arcaica, Barcelona: 677-769, 679-680.

Primavesi, O. (1969 [2008]) "Das Proömium des Parmenides und die Katabasis des Pythagoras", Phronesis 14, 1-30 (= Burkert in Kleine Schriften VIII - Philosophica edd. Th. A. Szlezák \& K.-H. Stanzel, Göttingen (Hypomnemata, Supplement, 2): 1-27.

Prozdnev, M. (2001) “De Parmenidis fragmento B 1,3 DK”, Hyperboreus 7 (1-2): 91-101.

Raubitscher, A. E. (1949) Dedications from the Athenian Acropolis, 1949.

Reale, G. (2004) Parmenide, Poema sulla natura, a cura di G. Reale, Milano.

Renehan E. (1992) "[Rec.] The Fragments of Parmenides by A. E. Coxon", Ancient Philosophy 12: 2 .

Riaux, F. (1840) Essai sur Parménide d'Elee, suivi du Texte et de la Traduction des Fragments, Paris.

Riezler, K (1934) Parmenides, Frankfurt.

Ritter, H.; Preller L. \& Wellmann E. (1913) Historia philosophiae Graecae, Gotha.

Santoro, F. (2011) Filósofos Épicos I: Parmênides e Xenófanes, fragmentos, Revisão Científica Néstor Cordero, Rio de Janeiro.

Santos, J. T. (1997 [2002]) Da Natureza. Parmênides. Lisboa, Alda (São Paulo, Loyola)

Sassi, M. M. (1988) "Parmenide al bivio. Per un'interpretazione del proemio", Parola del Passato 43: 387-357.

Sider, D. \& Johnstone H. (1986) The Fragments of Parmenides, Bryn Mawr.

Tarán, L. (1965) Parmenides. A Text with Translation, Commentary, and Critical Essays, Princeton University Press.

Tarrant, H. A. S. (1976) "Parmenides B.1.3: text, context and interpretation”, Antichton 10: 1-7. Tarrant, H. A. S. (2016) Parmenides' Vision: A Study of Parmenides' Poem, University Press of America.

Thanassas, P., (2007) Parmenides, Cosmos, and Being: A Philosophical Interpretation. Vol. 57: Marquette Univiversity Press.

Tortelli-Ghidini M. \& Scalera McClintock, G. (1990) "Aletheia nel pensiero orfico", Filosofia e Teologia 4: 73-84.

Untersteiner, M. (1958) Parmenide. Testimonianze e frammenti, Firenze.

Untersteiner, M. (1979) Parmenide. Testimonianze e Frammenti. Intr., trad. e comm. a cura di M. Untersteiner, Firenze.

Usener, H. (1889) "Variae lectiones specimen primum", Jahrbücher für classische Philologie 35. 
Ustinova, Y. (2009) Caves and the Ancient Greek Mind: Descending Underground in the Search for Ultimate Truth, Oxford University Press.

Verdenius, W. J. (1942) Parmenides. Some Comments on his Poem, Groningen.

Verdenius, W. J. (1949) “Parmenides' Conception of Light”, Mnemosyne 2: 119-120.

Vlastos, G. (2008) "Names of Being in Parmenides", Las Vegas.

Wilkinson, L. A. (2009) Parmenides and To Eon. Reconsidering Mythos and Logos, Continuum International Publishing Group, London-New York.

Zafiropoulo, J. (1950) L'école Eléate. Parménide, Zénon, Melissos, Paris.

Zucchiello, D. (2011) "Parmenide e la tradizione del pensiero del pensiero greco arcaico (ovvero della sua eccentricità)", in Studi di filosofia antica in onore di Livio Rossetti, a cura di S. Giombini \& F. Marcacci, Perugia: 165-178. 